\title{
SOLUTIONS TO SEQUENCING AND BARGAINING GAMES
}

Guangjing Yang 


\section{SOLUTIONS TO SEQUENCING AND BARGAINING GAMES}

GUANGJING YANG 



\section{SOLUTIONS TO SEQUENCING AND BARGAINING GAMES}

\section{DISSERTATION}

to obtain

the degree of doctor at the University of Twente, on the authority of the rector magnificus, prof. dr. ir. A. Veldkamp on account of the decision of the Doctorate Board, to be publicly defended on Wednesday 24th February 2021 at 12:45 hrs

by

\section{Guangjing Yang}

born on the 1st of April 1993

in Xiangyang, China 
This dissertation has been approved by the supervisors

prof. dr. M. Uetz, prof. dr. H. Sun and the co-supervisor dr. R. P. Hoeksma

The research reported in this thesis has been carried out within the framework of the MEMORANDUM OF AGREEMENT FOR A DOUBLE DOCTORATE DEGREE BETWEEN NORTHWESTERN POLYTECHNICAL UNIVERSITY, PEOPLE'S REPUBLIC OF CHINA AND THE UNIVERSITY OF TWENTE, THE NETHERLANDS

DSI Ph. D.Thesis Series No. 21-001

UNIVERSITY | DIGITAL SOCIETY Digital Society Institute

OF TWENTE. INSTITUTE $\quad$ P. O. Box 217, 7500 AE Enschede, The Netherlands.

ISBN: 978-90-365-5129-8

ISSN: 2589-7721 (DSI Ph.D. thesis Series No. 21-001)

DOI: $10.3990 / 1.9789036551298$

Available online at https://doi.org/10.3990/1.9789036551298

Typeset with HTEX

Printed by (To be filled), Enschede

Cover design by Guangjing Yang

Copyright (C2021 Guangjing Yang, Enschede, The Netherlands

All rights reserved. No part of this work may be reproduced, stored in a retrieval system, or transmitted in any form or by any means, electronic, mechanical, photocopying, recording, or otherwise, without prior permission from the copyright owner. 


\title{
Graduation Committee
}

Chairman/secretary:

\author{
prof. dr. J. N. Kok
}

University of Twente

Supervisors:

prof. dr. M. J. Uetz

University of Twente

prof. dr. H. Sun

Northwestern Polytechnical University

Co-supervisor:

dr. R. P. Hoeksma

University of Twente

Members:

prof. dr. F. Thuijsman

Maastricht University

prof. dr. J. R. van den Brink

VU University Amsterdam

prof. dr. J. L. Hurink

University of Twente

dr. A. Skopalik

University of Twente

dr. B. Manthey

University of Twente

prof. dr. G. Xu

Northwestern Polytechnical University 



\section{Preface}

This thesis has been written under the umbrella of the joint research institute on Networks, Economic Decisions and Optimization (NEDO) between the Northwestern Polytechnical University of Xian (NWPU, China) and the University of Twente (UT, The Netherlands). That means that part of the research underlying this thesis was done while I was a PhD student at NWPU, and part of it was done while being a PhD student at the UT, with prof. Hao Sun and prof. Marc Uetz as supervisors from NWPU and the UT, respectively. The thesis is based on the following set of research papers.

\section{Papers underlying this thesis}

[1] Cooperative sequencing games with position-dependent learning effect, Operations Research Letters 48-4 (2020), 428-434. (with H. Sun and M. Uetz).

(Chapter 3)

[2] Games in sequencing situations with externalities, European Journal of Operational Research, 278-2 (2019), 699-708. (with H. Sun, D. Hou and G. Xu).

(Chapter 4)

[3] A noncooperative bargaining game with endogenous protocol and partial breakdown, Mathematical Social Sciences 105 (2020), 34-40. (with with H. Sun, D. Hou and G. Xu).

(Chapter 5)

[4] A coalitional bargaining game with majority rule (manusrcipt). (with H. Sun, M. Uetz, R. P. Hoeksma, A. Skopalik).

(Chapter 6)

[5] Nash bargaining with rational threats in generalized partition function games (manusript). (with H. Sun, D. Hou and G. Xu).

(Chapter 7) 



\section{Contents}

Preface vii

1 Introduction 1

2 Notations and preliminaries $\quad 7$

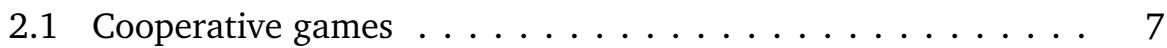

2.2 Noncooperative games . . . . . . . . . . . . . . 9

2.3 Sequencing situations $\ldots \ldots \ldots \ldots \ldots \ldots \ldots \ldots$

2.4 Partition function form games $\ldots \ldots \ldots \ldots \ldots$

3 Solutions to sequencing games with learning effect 17

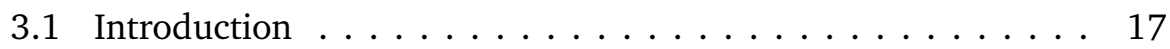

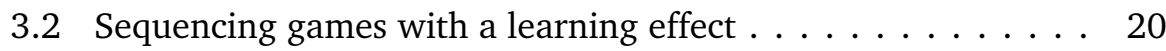

3.3 The family of LE-EGS rules and the $\Gamma$-rule . . . . . . . . . . . 29

3.3.1 The family of LE-EGS rules . . . . . . . . . . . . . . 29

3.3 .2 The $\Gamma$-rule . . . . . . . . . . . . . . . 30

3.3.3 A characterization of the $\Gamma$-rule . . . . . . . . . 35

3.3.4 Relationships between the $\Gamma$-rule and the $\beta$-rule . . . 39

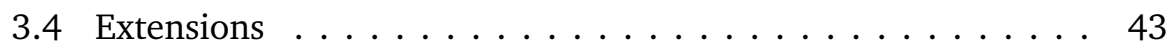

3.5 The PoA of noncooperative games for $\mathrm{LE}^{w}$ sequencing situations 44

3.5.1 The existence of stable orders . . . . . . . . . . 46

3.5.2 Lower and upper bound on the PoA . . . . . . . . . 48

3.6 Computational complexity of sequencing with learning . . . . 52

4 Games in sequencing situations with externalities 55 
4.1 Introduction $\ldots \ldots \ldots \ldots \ldots \ldots \ldots \ldots \ldots$

4.2 Partition sequencing games . . . . . . . . . . . . . . 59

4.3 Partition rules and the core $\ldots \ldots \ldots \ldots \ldots \ldots$

4.3.1 Partition rules . . . . . . . . . . . . . . . 72

4.3.2 Cores of partition sequencing games . . . . . . . 73

4.4 A noncooperative implementation of the EGS rule . . . . . . 76

5 Bargaining games with endogenous protocol and breakdown $\quad 83$

5.1 Introduction . . . . . . . . . . . . . . . . 84

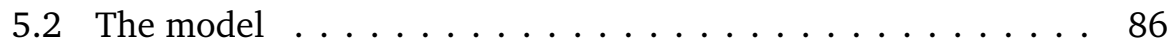

5.3 Main results $\ldots \ldots \ldots \ldots \ldots \ldots \ldots$

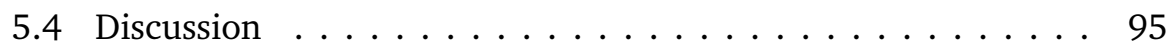

5.4.1 The implementation of the coalition structure core . . 95

5.4 .2 No-delay SSPE . . . . . . . . . . . . . . . . . 96

5.4.3 Related studies and Comparisons . . . . . . . . . . . 99

6 A coalitional bargaining game with majority rule 101

6.1 Introduction . . . . . . . . . . . . . . . . . 101

6.2 The model . . . . . . . . . . . . . . . . . . 103

6.3 The characterization and existence of SSPEs . . . . . . . . 105

6.4 Grand-coalition efficient games . . . . . . . . . . . . . 112

6.5 Existence of grand-coalition efficient games . . . . . . . . . 120

7 Nash bargaining in generalized partition function games $\quad 125$

7.1 Introduction . . . . . . . . . . . . . . . 125

7.2 The general and extended partition function games . . . . . 129

7.3 The Nash bargaining model . . . . . . . . . . . . . . 133

7.3 .1 The model . . . . . . . . . . . . . . . . 133

7.3 .2 Examples . . . . . . . . . . . . . . 137

7.3 .3 Uniqueness of RNBP . . . . . . . . . . . . . . . 138

7.4 Sufficient conditions for the existences of RTPPs . . . . . . . 140

7.5 The convexity and the core . . . . . . . . . . . . . 147

7.6 Concluding remarks . . . . . . . . . . . . . . . . . 149 
7.6.1 Comparisons between our games and PFF games . . . 149

7.6 .2 Alternative models . . . . . . . . . . . . . . . . 150

Summary 153

Bibliography 157

Acknowledgements 167

About the Author 169 



\section{Chapter 1}

\section{Introduction}

The main objective of this thesis is to explore the applications of game theory in certain sequencing and bargaining situations. Our major contribution is a study of stable solutions and equilibrium strategies in different scheduling and bargaining models.

Intuitively speaking, game theory is the study of using mathematical tools to analyze and model the interaction between rational decision-makers. It has a broad application field and offers insight into many economic, political or social situations that involve multiplayer strategy conflicts. The foundations of game theory were laid in the landmark book Theory of Games and Economic Behavior by von Neumann and Morgenstern [86]. Since then, game theory has been developed into a mature research area with many subfields and connections to other research areas such as computer science and mathematics. Traditionally, game theory can be divided into two different, but related branches: noncooperative game theory and cooperative game theory. Broadly speaking, the major difference is whether players can make binding contracts before the game starts. In noncooperative games, players' strategies are mutually independent. Each player tries to obtain his or her desirable outcome according to his or her preferences while anticipating, to some level, the strategic behavior of others. In cooperative games, binding agreements are made among players who are interested in coordinating their actions. 
To further illustrate the application of game theory, let us consider the following example.

Example 1.1. Foxconn, as one of the largest contract handset manufacturers, supplies assembly services for many customer electronics companies like Apple, Dell, and Huawei [88]. The sequence in which the orders of different customers are processed mostly relies on when Foxconn receives those orders. For the customers, waiting longer on the completion of an order induces higher costs.

The problem of finding the best sequence in which to process the orders can be approached in many different ways. In cooperative game theory, for example, a typical question would be if the customers can reach alliances, within which they can exchange their position in the sequence and reallocate costs, such that every customer is better off. In non-cooperative game theory, we are typically more interested in what the outcome of a game with a fixed set of rules could be. In this example, the rule could be that any two consecutive companies in the sequence can exchange their positions. Now we could ask ourselves whether equilibrium outcomes do exist when an equilibrium is defined as a situation where no two subsequent customers would have an incentive to switch their positions, and how bad such equilibria may be in comparison to the sequence that minimizes total waiting costs. Another problem in game theory is the question of whether certain rules for bargaining procedures could lead to desirable outcomes.

As illustrated by Example 1.1, even very simple economic settings can lead to questions both in noncooperative game theory and cooperative game theory by considering companies as players in the game. As a matter of fact, when we adopt the cooperative game theory, this example corresponds to an interesting problem in the research field on the frontier between cooperative game theory and operations research, which is called the sequencing game as introduced by Curiel et al. [21], and which was followed by an extensive literature, e.g., [23], [35], [38], and [80]. Indeed, Curiel et al. [21] allow players in the sequence to cooperate with each other by rearranging their positions. The worth of a coalition is the maximal cost savings its members can obtain. The central question, in cooperative game theory in general, is whether the 
core, which is arguably the most important solution concept in cooperative game theory (see, for example, [31] and [49]), is nonempty. That means if there are monetary transfers that could "enforce" the optimal sequence, in the sense that no coalition has an incentive to deviate from that solution. Curiel et al. [21] indeed introduce a cost savings allocation rule called the Equal Gain Splitting rule (EGS rule), which has these desirable properties.

Despite quite some work in the area of sequencing games, two situations, which are natural enough in the real world, had been ignored: (i) the learning effect, which is the phenomenon that the time it takes to process a product may decrease at some rate in the context of manufacturing. In position-dependent learning, for example, the later the position of a job in the schedule, the shorter its processing time is. This phenomenon typically matches realities where people and artificial intelligence have learning ability. Although the learning effect has not been considered in sequencing games, literature on this aspect can be found in the research field of scheduling (see, for example, [5], [6], [16], [87], and [90]). (ii) Externalities, which is also not covered in classical cooperative game theory until the second half of the twentieth century, state that the worth of a coalition depends not only on the behavior of its members, but also on the actions of all non-members. Cooperative games with externalities, also known as partition function form games, were first modeled in 1963 by Thrall and Lucas [83], and since then widely studied (see, for example, [36], [44], [52], and [53]). The foundational assumption in these games is that the worth of a coalition depends on the entire coalition structure and not just the coalition itself. Indeed, a major part of cooperative game theory assumes this situation away and is mainly based on the characteristic function, which assumes the worth of a coalition is independent of anything except the coalition itself. However, in bargaining processes, the coalitional influence reflects quite complex strategic interactions among players, and is still an interesting research topic. One of our contributions is to provide solutions for sequencing games when the learning effect and externalities are present. They are introduced in Chapter 3 and in Chapter 4.

As indicated above, one approach to solving problems like Example 1.1, is by a noncooperative bargaining process. A two-person bargaining problem 
was first proposed by Nash [65]. In the Nash bargaining game, two players need to agree on a division of some profit in a set $A$ of alternatives. If they reach an agreement on the division $a \in A$, then $a$ will be the final outcome. Otherwise, a fixed disagreement outcome $d$ will be the result. Nash [65] showed that there is a unique solution, called the Nash bargaining solution, satisfying several desirable properties. Rubinstein [75] also provided another well-known two-person bargaining model in which players take turns proposing a division of some profit. If the proposal is accepted, the proposed division will be the final result. Otherwise, the game goes on, and the future payoffs for both players will be decreased at some discount rate. Rubinstein [75] proved that there is a unique subgame perfect equilibrium converging to the Nash bargaining solution if the discount rate converges to 1 . Since the pioneering works of Nash and Rubinstein, noncooperative bargaining games have been extensively studied from different perspectives in the literature. For example, Okada [69] studied the Nash bargaining solution in general $n$-person cooperative games. Chatterjee et al. [15] extended the Rubinsteintype two-person bargaining game to the $n$-person barganing game. Following the research of Chatterjee et al. [15], Okada $[68,70]$ presented coalitional bargaining games with random proposers. Bloch [7], Gomes [32], and also Ray and Vohra [73] studied noncooperative bargaining games where externalities exist.

In this thesis, we present a sequential bargaining model for sequencing situations with externalities in Chapter 4. We show that the unique subgame perfect equilibrium is general enough to implement the EGS rule. Another contribution is to study noncooperative bargaining games in more general (not limited to sequencing) situations for stable allocation schemes and possible relationships between equilibria and other solution concepts in cooperative game theory. We use a noncooperative sequential bargaining game to show that stationary subgame perfect equilibria of this game coincide with the core. In order to further extend noncooperative bargaining games, we follow Okada [68] and Ray and Vohra [73] by considering the majority rule in every responding phase of the game. We show that every game of this kind has a stationary subgame perfect equilibrium. In addition, we study the Nash bargaining solution in a general partition function form game. 
In summary, this thesis contains 5 research chapters as well as this introductory chapter and a chapter on notations and definitions. The research chapters can be read more or less self-contained. In the remainder of this chapter, we give a brief overview of the contents of the research chapters.

Chapter 3 extends sequencing games as introduced by Curiel et al. [21] to the setting with a position-dependent learning effect. We first show that these games are balanced, and analyze the family of equal gains sharing (EGS) rules. Then, we show that, in contrast to games without learning effect, only a specific class of EGS rules leads to core allocations. This allocation rule is characterized axiomatically, and we also study its relationship with the $\beta$-rule, earlier introduced by Curiel et al. [23]. Finally, a noncooperative game for the sequencing situations where players have different weights is provided, of which the price of anarchy is studied.

In Chapter 4, we consider cooperative games in sequencing situations with externalities, called partition sequencing games. In these games, the worth of a coalition can be influenced by external players in the queue. We first show that partition sequencing games satisfy cohesiveness and have nonpositive externalities. Then by proposing partition rules, which are specifications on how external players should partition themselves, we study the relationships between the allocation given by the EGS rule and the core based on different partition rules. We show that the EGS rule always yields a core element independent of the partition rule that is applied to the game. Moreover, we propose a mechanism that implements the EGS rule for partition sequencing games.

In Chapter 5, we present a noncooperative sequential bargaining game with transferable utility. Instead of assuming that the recognition of proposers is determined randomly or by a fixed exogenous protocol, we provide a mechanism in which the protocol is generated endogenously. Besides, we consider a partial breakdown probability rather than a discount factor when the proposal is rejected by some player. We show that for each partial breakdown probability, stationary subgame perfect equilibria exist if the characteristic function game is totally balanced. Moreover, the outcomes of stationary subgame perfect equilibria in our model coincide with the core allocations. 
Chapter 6 proposes a noncooperative "legislative" bargaining model in which each legislator has several votes and the approval of a proposal requires only a fraction of the approval committees' votes. We first provide the characterization of the set of stationary subgame perfect equilibria. Then with the help of this characterization, we show the existence of these equilibria. Moreover, when we restrict our attention to the class of grand-coalition efficient games, we show that the pivotal players extract all the gains from the grand coalition if their discount rates go to 1. Finally, we also elaborate on the existence of these grand-coalition efficient games.

In Chapter 7, we propose a class of games, called general partition function games (GPFG), in which externalities across coalitions are considered. In order to obtain a reasonable worth for each coalition in these games, the Nash bargaining model is employed and we show that there exists at most one rational Nash bargaining payoff (RNBP) for any deviating coalition. We provide two sufficient conditions for the existences of rational threat partition pairs (RTPPs), which are essential for the determination of RNBP. Moreover, by viewing the RNBP as the worth of a deviating coalition, we define the core of the corresponding characteristic function form game. We further provide two sufficient conditions for the non-emptiness of the core. 


\section{Chapter 2}

\section{Notations and preliminaries}

\subsection{Cooperative games}

Definition 2.1. A cooperative game is a pair $(N, v)$, where $N$ is a nonempty, finite set and $v: 2^{N} \rightarrow \mathbb{R}$ is a characteristic function satisfying $v(\emptyset)=0$.

The set $N$ is called the grand coalition. An element of $N$ (notation: $i \in N$ ) and a subset $S$ of $N$ (notation: $S \in 2^{N}$ with $S \neq \emptyset$ ) are called a player and coalition respectively. The associated real number $v(S)$ is called the worth of coalition $S$. Given any coalition $S,\left(S, v_{S}\right)$ is a subgame of a cooperative game $(S, v)$ if for every $T \subseteq S, v_{S}(T)=v(T)$.

A cooperative game $(N, v)$ always contains the information about how much each coalition can produce by cooperating with its members. The characteristic function $v$ of any cooperative game $(N, v)$ has an implicit underlying assumption: the worth of a coalition $S$ does not depend on the players not in $S$ nor on the coalition structure outside $S$. A cooperative game can be also called a characteristic function form game. However, we will see that this assumption is removed in a more general model, which is called the partition function form game and will be introduced in Subsection 2.4.

We now introduce some basic definitions for cooperative games.

Definition 2.2. A cooperative game $(N, v)$ is called superadditive if $v(S)+$ $v(T) \leq v(S \cup T)$ for any $S, T \in 2^{N}$ with $S \cap T=\emptyset$. 
In a superadditive cooperative game, what the union of two disjoint coalitions can obtain is at least the sum of worths when they work separately.

Definition 2.3. A cooperative game $(N, w)$ is strategically equivalent to another cooperative game $(N, v)$ if there exist a positive number $a$ and $n$ arbitrary real numbers $b_{i}, i \in N$, such that for every $S \subseteq N$,

$$
w(S)=a v(S)+\sum_{i \in S} b_{i}
$$

Definition 2.4. A cooperative game $(N, v)$ is $(0,1)$-normalized if for every player $i \in N, v(\{i\})=0$ and $v(N)=1$.

It is well-known that a cooperative game is strategically equivalent to a $(0,1)$-normalized game if and only if it is essential ${ }^{1}$ (see [55]). In general, any essential game can be transformed into a $(0,1)$-normalized game by changing the units of measurement and adding a constant value for each coalition.

Definition 2.5. Given any nonempty coalition $T \subset N$, the game $\left(N, u_{T}\right)$ is a unanimity game if

$$
u_{T}(S)= \begin{cases}1 & \text { if } T \subseteq S, \\ 0 & \text { otherwise }\end{cases}
$$

In other words, a unanimity game $\left(N, u_{T}\right)$ is a cooperative game in which a coalition cannot obtain its worth unless all players in $T$ are contained in that coalition. In the bargaining context, it means that a proposal is approved only if all players in $T$ accept it.

We next introduce a very important solution concept in cooperative game theory, which is called the core and first proposed by Gillies [31].

Definition 2.6. The core of a cooperative game $(N, v)$ is defined by

$$
C(N, v)=\left\{x \in \mathbb{R}^{n} \mid x(N)=v(N), x(S) \geq v(S) \text { for all } S \subseteq N\right\},
$$

where $x(S)=\sum_{i \in S} x_{i}$.

\footnotetext{
${ }^{1} \mathrm{~A}$ cooperative game $(N, v)$ is essential if $v(N)>\sum_{i \in N} v(\{i\})$.
} 
The core is the set of efficient allocations of $v(N)$ such that there is no coalition with an incentive to split off from the grand coalition. The core of a game can be empty and a game that has a nonempty core is called balanced. A game $(N, v)$ is totally balanced if and only if the core of every subgame of $(N, v)$ is nonempty.

Definition 2.7. A game $(N, v)$ is said to be convex if

$$
v(T \cup\{i\})-v(T) \geq v(S \cup\{i\})-v(S)
$$

for every $i \in N$ and for every $S \subseteq T \subseteq N \backslash\{i\}$.

It has been shown by Shapley [78] that the core of a convex cooperative game is nonempty.

\subsection{Noncooperative games}

In this section, we first introduce the noncooperative games in extensive form (for noncooperative games in strategic form, see [55]).

Definition 2.8. A noncooperative game in extensive form can be represented by a 6 -tuple $(N, e, G, P, O, u)$, where

- $N$ is the set of players, and $e$ is nature.

- $G$ is a rooted directed tree, which is called the game tree. Each vertex $d$ of $G$ represents a state that the game can be in. A vertex $d$, at which a player $i \in N$ is about to make a decision, is called the vertex of player $i$. For each player $i$, every edge from his or her vertex to one of the vertex's children is called an action of player $i$. The set of all actions of player $i$ is denoted by $A_{i}$.

- $O$ is the set of all possible outcomes of the game. Each leaf of $G$ represents one outcome in $O$.

- $P=\left\{P_{i}\right\}_{i \in N \cup\{e\}}$ is a partition of subsets of all vertices of $G$, except for the set of leaves of $G$, into $n+1$ subsets, one subset $P_{i}$ for each player 
$i$ and one subset $P_{e}$ for nature $e$. For all $i \in N, P_{i}$ denotes the set of all information sets of player $i$. Letting $P_{i}=\left\{D_{i}^{1}, \cdots, D_{i}^{k}\right\}$, each subset $D_{i}^{j} \in$ $P_{i}$ is called an information set of player $i$, and $D_{i}^{j}$ contains some vertices of player $i$ that have the same parent. Any information set $D_{i}^{j}$ of a player $i$ containing more than 1 vertex means that player $i$ has information about that the game is in a state represented by a vertex of $D_{i}^{j}$, but player $i$ is not aware which vertex it is.

- Mapping $u$ is a function that maps every outcome $o \in O$ to a vector $u(o) \in \mathbb{R}^{n}$. The vector $u(o)$ is called the payoff vector, where for each player $i, u_{i}(o)$ is the payoff for player $i$ in outcome $o$.

In the above definition, nature is usually taken as a fictional player and represents some external factor that could influence the game. For example in Chapter 6, the selection of the proposer in the bargaining game can be regarded as a random decision of nature. A noncooperative game in extensive form is called a game with perfect information if every information set of every player contains exactly one vertex. In general words, when we deal with a game with perfect information, a player who is about to make a decision always knows exactly the current state that the game is in. Throughout this thesis, all noncooperative games in extensive form are games with perfect information.

We next give the definition for subgames of a noncooperative game in extensive form.

Definition 2.9. A subgame ( $N, e, G(d), P(d), O, u)$ of a noncooperative game in extensive form $(N, e, G, P, O, u)$ is the game where

- $N$ is the set of players and $e$ is nature as in the game (N,e, G, P,O,u).

- $G(d)$ is a sub-tree of $G$, where $d$ is a vertex of $G$ and the root of $G(d)$. The sub-tree $G(d)$ contains all vertices of $G$ that are descendants of $d$ in the game tree $G$.

- $P(d)=\left\{P_{i}(d)\right\}_{i \in N \cup\{e\}}$ is a partition of subsets of all vertices in $P(d)$, where $P_{i}(d)=P_{i} \cap G(d)$ is the set of information sets of player $i$ in $G(d)$. 
- $O$ is the set of all possible outcomes as in $(N, e, G, P, O, u)$ restricted to the set of leaves in $G(d)$

- Mapping $u$ is the same function as in $(N, e, G, P, O, u)$, restricted to the set of leaves in $G(d)$

Definition 2.10. For each player $i$, let $D_{i}^{j}$ be one of his or her information sets. A strategy for player $i$ is a function $s_{i}$ that maps from each information set $D_{i}^{j}$ to an action $a_{i} \in A_{i}$.

Definition 2.11. Let $S_{i}$ be the set of all strategies for player $i$. A mixed strategy is a function $\eta_{i}: S_{i} \rightarrow[0,1]$, and $\sum_{s_{i} \in S_{i}} \eta_{i}\left(s_{i}\right)=1$.

Let $\mathscr{D}_{i}$ be the collection of all information set of player $i$, and $A\left(D_{i}^{j}\right)$ be the set of all possible actions at $D_{i}^{j}$. For any set $E$, let $\Delta(E)$ be the set of all probability distributions over $E$.

Definition 2.12. A behavior strategy for player $i$ is a function $\alpha_{i}: \mathscr{D}_{i} \rightarrow$ $\bigcup_{D_{i}^{j} \in \mathscr{D}_{i}} \Delta\left(A\left(D_{i}^{j}\right)\right)$.

In general words, if a player $i$ plays a strategy $s_{i}, s_{i}$ includes instruction for the player $i$ on how to behave at each his or her vertex in the game tree. The mixed strategy $\eta_{i}$ gives each strategy $s_{i}$ a probability $\eta_{i}\left(s_{i}\right)$, which represents the possibility for player $i$ to choose $s_{i}$. The behavior strategy $\alpha_{i}$ for player $i$ is a function that assigns each information set $D_{i}^{j}$ of player $i$ a probability distribution over $A\left(D_{i}^{j}\right)$. The probability distribution $\alpha_{i}\left(D_{i}^{j}\right)$ assigns each possible action $a_{i}$ at $D_{i}^{j}$ a probability, which is denoted by $\alpha_{i}\left(a_{i} ; D_{i}^{j}\right)$.

Let $\eta_{i}$ or $\alpha_{i}$ be a mixed or behavior strategy for player $i$. The strategy vector $\eta=\left(\eta_{1}, \cdots, \eta_{n}\right)$ or $\alpha=\left(\alpha_{1}, \cdots, \alpha_{n}\right)$ is called a (mixed or behavior) strategy profile. It is known that a mixed strategy profile $\eta$ and a behavior strategy profile $\alpha$ are equivalent to each other if for every vertex $d$ in the game tree, the probability of visiting $d$ is equal from playing $\eta$ and $\alpha$. Because of their equivalence, throughout this work, if there is no confusion, we use the symbol $\eta$ to represent both the mixed strategy and behavior strategy.

Let $u(\eta)=\left(u_{1}(\eta), \cdots, u_{2}(\eta)\right)$ be the payoff vector if strategy profile $\eta$ is implemented, and $\eta_{-i}$ be a strategy profile for all players excpet for player $i$. 
Definition 2.13. A strategy profile $\eta$ is a Nash equilibrium if for every player $i \in N$, changing the strategy $\eta_{i}$ will not make player $i$ better off, i.e.,

$$
u\left(\eta_{i}, \eta_{-i}\right) \geq u\left(\eta_{i}^{\prime}, \eta_{-i}\right)
$$

for every alternative strategy $\eta_{i}^{\prime}$ of player $i$.

In Definition 2.13, $\eta_{i}$ is called a best response of player $i$ to other players' strategy profile $\eta_{-i}$. Note that the best response may not be unique. Let $u(\eta ; d)$ be the payoff restricted to the strategy profile $\eta$ and the subgame ( $N, e, G(d), P(d), O, u)$. We next give the definition of the subgame perfect equilibrium.

Definition 2.14. Let $(N, e, G, P, O, u)$ be a noncooperative game in extensive form. A strategy profile $\eta$ is a subgame perfect equilibrium (SPE) if for every subgame ( $N, e, G(d), P(d), O, u)$ of the original game ( $N, e, G, P, O, u)$, every player $i \in N$, and every alternative strategy $\eta^{\prime}$ of player $i$,

$$
u\left(\eta_{i}, \eta_{-i} ; d\right) \geq u\left(\eta_{i}^{\prime}, \eta_{-i} ; d\right)
$$

The subgame perfect equilibrium is a refinement of the Nash equilibrium. It represents a Nash equilibrium for every subgame of the original game. Note that every subgame perfect equilibrium is a Nash equilibrium, but the converse is not necessarily true.

\subsection{Sequencing situations}

In a sequencing situation, there is a queue of $n$ players, each of whom owns a single job that has to be processed on a machine. Player and job will be used interchangeably. The finite set of players is denoted by $N$ where $|N|=n$. A processing order or order in short, on the players is defined by a bijection $\sigma$ : $N \rightarrow\{1, \ldots, n\}$, where $\sigma(i)=j$ means that player $i$ is in position $j$. The initial given order is denoted by $\sigma_{0}$ and the set of all orders on $N$ is denoted by $\Sigma_{N}$. For every $i \in N$, player $i$ has a processing time $p_{i}$ and a weight $w_{j}$. A sequencing situation can be denoted by a 4-tuple $\left(N, \sigma_{0}, w, p\right)$. 
We call a coalition $S \subseteq N$ connected with respect to $\sigma \in \Sigma_{N}$ if for all $i, j \in S$ and $k \in N$ such that $\sigma(i)<\sigma(k)<\sigma(j)$ it holds that $k \in S$. For any connected coalition $S$ with respect to $\sigma$, we define $f_{S}^{\sigma}$ the first member within $S$ and $l_{S}^{\sigma}$ the last member within $S$, i.e.,

$$
f_{S}^{\sigma}=\arg \min _{i \in S} \sigma(i) \text { and } l_{S}^{\sigma}=\arg \max _{i \in S} \sigma(i) .
$$

A $\sigma$-component of $S$ is a maximally connected subset of $S$ with respect to $\sigma$. Given a coalition $S, S / \sigma$ denotes the set of $\sigma$-components of $S$. Finally, the set of coalitions that are connected with respect to $\sigma$ is denoted by $\operatorname{con}(\sigma)$.

The set of predecessors of player $j$ with respect to the order $\sigma$ is denotedd by $P(\sigma, i)=\{j \in N \mid \sigma(j)<\sigma(i)\}$ and the set of successors of player $i$ with respect to $\sigma$ is defined by $F(\sigma, i)=\{j \in N \mid \sigma(j)>\sigma(i)\}$. We define $\bar{P}(\sigma, i)=$ $P(\sigma, i) \cup i$ and $\bar{F}(\sigma, i)=F(\sigma, i) \cup i$. Given $\sigma_{0}$, a processing order $\sigma \in \Pi_{N}$ is called admissible for $S$ if it satisfies the following condition,

$$
P\left(\sigma_{0}, j\right)=P(\sigma, j) \text { for all } j \in N \backslash S \text {. }
$$

This means that only players inside $\sigma_{0}$-components of $S$ are allowed to exchange their relative positions with each other, and in particular, all players outside $S$ remain at the same positions as in $\sigma_{0}$. The set of all admissible orders for a coalition $S$ is denoted by $\Sigma_{S}$.

An SPT (shortest processing time first) order is the order in which the jobs are arranged according to non-decreasing processing times. A WSPT (weighted smallest processing time first) order is the order in which the jobs are arranged according to non-decreasing sequence of $\frac{p_{i}}{w_{i}}$. Denote by $\sigma_{S}$ the order which is attained from $\sigma_{0}$ by reordering the members in each $\sigma_{0}$ component of $S$ with respect to the SPT order, i.e., (i) $\sigma_{S}(i)=\sigma_{0}(i)$ for every $i \in N \backslash S$, and (ii) $\sigma_{S}(i)<\sigma_{S}(j)$ for every $i, j \in T$ and every $T \in S / \sigma_{0}$ such that $p_{i}<p_{j}$.

Definition 2.15. Let $\sigma_{0} \in \Pi_{N}$. A cooperative game $(N, v)$ is called $\sigma_{0^{-}}$ component additive if it satisfies the following three conditions:

(i) $v(i)=0$ for all $i \in N$, 
(ii) $(N, v)$ is superadditive, and

(iii) $v(S)=\sum_{T \in S / \sigma_{0}} v(T)$ for all $S \in 2^{N}$.

Le Breton et al. [54] showed that $\sigma_{0}$-component additive games are always balanced, that is, have a nonempty core.

Curiel et al. [21] introduced a solution on how to allocate the total maximum cost savings among players. They call this solution the equal gain splitting (EGS) rule, which is defined by

$$
E G S_{i}=\frac{1}{2} \sum_{j: \sigma_{0}(j)>\sigma_{0}(i)} g_{i j}+\sum_{k: \sigma_{0}(k)<\sigma_{0}(i)} g_{k j},
$$

where $g_{i j}=\max \left\{0, p_{i} w_{j}-p_{j} w_{i}\right\}$. The EGS rule divides the cost savings obtained in a neighbor switch equally among the two players involved in the neighbor switch. It has been shown by Curiel et al. [21] that the EGS rule always yields a core element.

\subsection{Partition function form games}

Let $N=\{1, \ldots, n\}$ be a set of players. A partition of $N$ is defined by $\rho=$ $\left\{\emptyset, S_{1}, \ldots, S_{k}\right\}$, where $S_{i} \neq \emptyset$ for $i \in\{1, \ldots, k\}, S_{i} \cap S_{j}=\emptyset$ for $i, j=1, \ldots, k(i \neq j)$, and $\bigcup_{i=1}^{k} S_{i}=N$. If there is no danger of confusion, given any partition, we simply write $\left\{S_{1}, \ldots, S_{k}\right\}$ instead of $\left\{\emptyset, S_{1}, \ldots, S_{k}\right\}$. Let $\Pi_{N}$ be the set of all partitions of $N$. Given a coalition $S \subseteq N$, we denote by [S] the partition of $S$ into singletons and by $|S|$ the cardinality of $S$. In characteristic function form games, any coalition $S \subseteq N$ is associated with a real number $w(S)$, which denotes the worth of coalition $S$ and is independent of external players. However, in partition function form (PFF) games, the worth of a coalition may be affected by other coalitions. Formally, given any $\rho \in \Pi_{N}$ and any $S \in \rho$, the pair $(S ; \rho)$ is called an embedded coalition. The set of all embedded coalitions $\left\{(S ; \rho) \mid S \in \rho, \rho \in \Pi_{N}\right\}$ is denoted by $E C(N)$.

Definition 2.16. A PFF game is a pair $(N, v)$, where $v: E C(N) \rightarrow \mathbb{R}$ is a partition function, which assigns a value $v(S ; \rho)$ to each embedded coalition $(S ; \rho)$, for all $\rho$. By convention, $v(\emptyset ; \rho)=0$ for any $\rho$. 
We next provide some basic definitions of PFF games.

Definition 2.17. A PFF game is said to be cohesive if for every partition $\rho$ of $N$,

$$
v(N ;\{N\}) \geq \sum_{S \in \rho} v(S ; \rho)
$$

The cohesiveness means that forming the grand coalition always generates the largest total surplus.

Definition 2.18. A PFF game is said to have non-positive externalities if for any mutually disjoint coalitions $S, T, U \subseteq N$, and for any partition $\rho$ of $N \backslash(S \cup$ $T \cup U)$,

$$
v(S ;\{S, T \cup U\} \cup \rho) \leq v(S ;\{S, T, U\} \cup \rho) .
$$

A PFF game having non-positive externalities means that a merger between two disjoint coalitions does not make other coalitions better off. 



\section{Chapter 3}

\section{Solutions to sequencing games with learning effect}

This chapter extends sequencing games as introduced by Curiel et al. [21] to the setting with a position-dependent learning effect. We first show that these games are balanced, and analyze the family of equal gains sharing (EGS) rules. Then, we show that, in contrast to games without learning effect, only a specific class of EGS rules leads to core allocations. This allocation rule is characterized axiomatically, and we also study its relationship with the $\beta$-rule, earlier introduced by Curiel et al. [23]. Finally, a noncooperative game for the sequencing situations where players have different weights is provided, of which the price of anarchy is studied.

\subsection{Introduction}

Research on the intersection of cooperative game theory and scheduling was initiated by Curiel et al. [21]. They introduced a class of cooperative games called sequencing games. They arise from the following single machine sequencing problem: there is one single machine set up to process a finite number of $n$ jobs, together with an initial given order of these jobs. Each job has a processing time and is owned by one agent, and each agent is interested in 
minimizing its own job's completion time. Since the initial order is usually not the optimal one for minimizing the total costs of all players, any coalition of agents can in principle realize cost savings by changing their relative positions in the schedule. In this situation, one of the fundamental questions in cooperative sequencing games is how the globally optimal solution can be supported by redistributing the total cost savings among the agents so that no coalition has an incentive to deviate from it. These stable allocations are precisely the core of the underlying cooperative game.

Curiel et al. [21] showed that such sequencing games are convex and therefore core allocations exist. They also introduced and characterized the equal gain splitting (EGS) rule, which can be interpreted as an algorithmic procedure to compute a core allocation. During the past few decades, different types of variations of sequencing situations and games have been presented in the literature, including ready times [38], due dates [9], multiple machines [12, 39, 80], grouped jobs [17, 35] multistage situations $[18,19]$ and sequencing situations without an initial order [51].

A common assumption in all of the above works is that the processing times of jobs are constants and independent of their positions during the scheduling process. However, it is a common assumption that facilities such as workers, or computers augmented with artificial intelligence can improve their performance over time, by processing jobs. As a result, the processing time will be shorter if a job is scheduled in a later position. This phenomenon is well known in the literature as a "learning effect", which was introduced e.g. by Biskup [5] who considers the actual processing time of a job as a decreasing power function of its position in the schedule.

The purpose of this chapter is to extend cooperative sequencing games to the situation where a position-dependent learning effect exists, meaning that the processing time of a job also depends on the number of jobs that are scheduled before it. We call these games LE (learning effect) sequencing games. The question that we ask is whether the results obtained by Curiel et al. [21] for (ordinary) sequencing games will still hold in situations with a learning effect. In brief, the answer is yes, but with some non-trivial extensions and modifications of earlier ideas and proof techniques. Let us next sketch the major contributions of this chapter. 
For sequencing games, Curiel et al. [21] defined the worth of a coalition as the maximal cost savings it can obtain by admissible reordering the members of this coalition. However, a problem arises if we adopt this definition to LE sequencing games: the non-members who are placed later than the coalition in consideration will also benefit from the cooperation of this coalition, that is, their completion times will be reduced. We propose, in Section 3.2, how the worth of a coalition can be defined in LE sequencing games. How much of the "external" benefits of cooperative behavior should be allocated to the cooperative coalition, will be measured by a share function, introduced in Section 3.2. We show, maybe unsurprisingly, that the resulting LE sequencing games are balanced, hence have a nonempty core. In particular, LE sequencing games in which the cooperating coalition obtains the full benefits of cooperation, are even convex.

In Section 3.4, we then analyze how core allocations can be computed through adjacent exchanges of positions and EGS allocation rules. The main difficulty, in contrast to earlier works, lies in the fact that the cost savings contributed by an adjacent exchange does not only depend on the processing times of the two players involved, but also on the positions where these two players are in the schedule. This means that the outcomes could differ depending on the orders of neighbor switches. Unlike with Curiel et al. [21], who showed that EGS rules always yield a core allocation no matter in which order the adjacent exchanges are realized, we show that not all LE-EGS rules lead to core elements for LE sequencing games. Interestingly, though, we identify one particular order of adjacent exchanges, and prove that it ensures that the corresponding LE-EGS rule yields a core element. This particular allocation rule is called the $\Gamma$-rule, and we provide its axiomatic characterization. We finally discuss the relationship between the $\Gamma$-rule and the $\beta$-rule that was earlier introduced by Curiel et al. [23].

In Section 3.5, we discuss two possible extensions of LE sequencing games: the $\mathrm{LE}^{a}$ and $\mathrm{LE}^{w}$ sequencing games, in which players may have different learning effects and different weights. However, since there is no fixed principle for the optimal orders in the two extensions, it is still an open problem how to bring the EGS rule into those games. In $L E^{w}$ sequencing situations, it is even unclear if there exists an algorithm with polynomial computation time for 
computing the optimal order. Therefore, in Section 3.6, we focus on different questions: motivated by the EGS rules, we address questions about the existence and quality of sequences that are "stable" in the following sense. We call a sequence stable if no two neighboring players have an incentive to switch positions, given that they could share the cost savings of the switch a positive exchange. We show that there must be at least one stable order in which players will no longer select neighbors for switching. Moreover, by analyzing the price of anarchy of the noncooperative games, we show that these games are relatively efficient when the number of players is small or the learning effect has little influence on the processing times. We end this chapter with a brief discussion about the computational complexity of finding optimal orders in $\mathrm{LE}^{w}$ sequencing situations in Section 3.6.

\subsection{Sequencing games with a learning effect}

In a sequencing situation with learning effect, the machine has the ability to improve (by processing jobs). As a result, the later a job is scheduled in the sequence of jobs, the shorter its processing time. We assume that each player $i \in N$ has a nominal processing time $p_{i}$. Given an order $\sigma \in \Pi_{N}$, the actual processing time of any job $i$ decreases as a function of its position, and it equals

$$
\sigma(i)^{a} p_{i}
$$

where $a \leq 0$ is the so-called learning index [5]. In this chapter, we assume that there is no idle time between jobs. Hence, the completion time of player $i$ is

$$
C(\sigma, i)=\sum_{\sigma(j) \leq \sigma(i)} \sigma(i)^{a} p_{i}
$$

which equals the cost of a job $i$ under sequence $\sigma$. In other words, the cost of a job equals the time it spends in the system.

Define an $L E$ sequencing situation by a 4-tuple $\left(N, \sigma_{0}, p, a\right)$, where $N=$ $\{1, \ldots, n\}$ is the set of $n$ players, $\sigma_{0} \in \Pi_{N}$ the initial order on the jobs, $p=$ $\left(p_{i}\right)_{i \in N} \in \mathbb{R}_{+}^{n}$ the vector representing the nominal processing times, and $a$ the learning index. 
Let $\left(N, \sigma_{0}, p, a\right)$ be an LE sequencing situation. A pair $(i, j)$ is inverted in $S$ if $i, j \in S, \sigma_{0}(i)<\sigma_{0}(j)$, and $p_{i}>p_{j}$. Given a connected coalition $S \subseteq N$, let us denote by $I^{S}$ the number of all inverted pairs from $S$ with respect to $\sigma_{0}$. An adjacent exchange is a mapping $\tau: \Pi_{N} \rightarrow \Pi_{N}$, and we denote the set of all adjacent exchanges by $\Lambda_{n}$. W.l.o.g. we can overload notation and assume that $\tau$ exchanges the jobs on positions $\tau$ and $\tau+1$ (i.e., we simply represent elements of $\Lambda_{n}$ by the first position $\tau$ of the adjacent exchange). To be precise, given $\sigma \in \Pi_{N}$, if $\tau(\sigma)=\sigma^{\prime}$, we have $\sigma^{-1}(\tau)=\sigma^{\prime-1}(\tau+1)$, $\sigma^{-1}(\tau+1)=\sigma^{\prime-1}(\tau)$, and $\sigma^{-1}(k)=\sigma^{\prime-1}(k)$ for any $k \neq \tau, \tau+1$.

The difference in total costs before and after an adjacent exchange $\tau$ on order $\sigma$ can be easily calculated. If we assume that $\sigma(i)=\tau$ and $\sigma(j)=\tau+1$, it will later be convenient to refer to this difference as $g(\tau, \sigma, i, j)$. It equals

$$
g(\tau, \sigma, i, j):=\left(p_{i}-p_{j}\right)\left[(n-\tau+1)(\tau)^{a}-(n-\tau)(\tau+1)^{a}\right] .
$$

Notice that the cost savings obtained by an adjacent exchange depend not only on the processing times of the two jobs $i$ and $j$, but also on the position $\tau$ of the adjacent exchange. The earlier the position, the larger the cost savings. For later use, it is convenient if we define

$$
\theta(\tau):=\left[(n-\tau+1)(\tau)^{a}-(n-\tau)(\tau+1)^{a}\right]
$$

Any order $\sigma$ can be obtained from any $\sigma_{0}$ by successive adjacent exchanges. We define a permutation process as an ordered set of adjacent exchanges $P e=\left\{\tau_{1}, \ldots, \tau_{m}\right\}, \tau_{1}, \ldots, \tau_{m} \in \Lambda_{n}$ such that $\tau_{1}\left(\sigma_{0}\right)=\sigma_{1}, \tau_{2}\left(\sigma_{1}\right)=$ $\sigma_{2}, \ldots, \tau_{m}\left(\sigma_{m-1}\right)=\sigma$. Let us write $P e\left(\sigma_{0}\right)=\sigma$ if permutation process $P e$ ends in $\sigma$. Moreover, recall that $\sigma_{S}$ is the order attained from $\sigma_{0}$ by reordering the members in each $\sigma_{0}$-component of $S$ in SPT order, and $I^{S}$ is the number of inverted pairs in $S$ with respect to $\sigma_{0}$. Let us call a permutation process feasible if $\mathrm{Pe}\left(\sigma_{0}\right)=\sigma_{S}$ and $|P e|=I^{S}$. That is, Pe successively exchanges inverted pairs of $S$. Finally, by $\mathscr{P}\left(\sigma_{0}, \sigma_{S}\right)$ we denote the set of all feasible permutation processes for $S$ and $\sigma_{0}$.

For any order $\sigma \in \Pi_{N}$, the total costs of all players with respect to $\sigma$ is $\sum_{i \in N} C(\sigma, i) . \sigma$ is called optimal if the total costs of all players with respect 
to $\sigma$ are minimized. Biskup [5] proved that if the jobs are arranged according to non-decreasing nominal processing times, i.e. in SPT order, the total costs of all players are minimal.

The following example shows that, in LE sequencing situations (with given $\sigma_{0}$ ), even if $\sigma \in \Pi_{S}$, that is, $\sigma$ is admissible for coalition $S$, player set $S$ may have an "external" effect also on players outside $S$, because of the learning effect.

Example 3.1. Let $N=\{1,2,3\}, \sigma_{0}=(1,2,3), p=\{3,2,1\}$, and $a=-1$. Consider the coalition $S=\{1,2\}$. If players 1 and 2 are willing to switch their positions, the total costs of coalition $\{1,2\}$ can be reduced by 1.5. At the same time, this switch decreases the completion times of $\{1,2\}$ by 0.5 . Now, because there is no idle time, player 3 enjoys cost savings of 0.5 , too.

Consequently, the question arises if and how much of these "external" cost savings should be attributed to coalition $S$ ? We suggest to capture this issue by defining a share function $\lambda: 2^{N} \rightarrow[0,1]$. This mapping represents a "tax rate" that is imposed by coalition $S$, on the members outside $S$. That said, we can fully define an $L E$ sequencing game $\left(N, v^{\lambda}\right)$ by defining the worth of a coalition $S \subseteq N$ by

$$
v^{\lambda}(S)=\max _{\sigma \in \Pi_{S}}\left\{\sum_{i \in S} \Delta C(\sigma, i)+\lambda(S) \sum_{j \in N \backslash S} \Delta C(\sigma, j)\right\},
$$

where $\Delta C(\sigma, i)=C\left(\sigma_{0}, i\right)-C(\sigma, i)$. In particular, if $\lambda(S)=0$ for all $S \subseteq N$, the definition of the worth of coalitions in LE sequencing games concurs with those of earlier works. For notational simplicity, in the following we denote by $(N, v)$ the LE sequencing games when $\lambda(S)=1$ for all $S \subseteq N$.

We will show by the next theorem that the order that achieves the maximal cost savings, and hence defines the worth of a coalition $S$, is exactly the SPT order. First we need the following lemma given by Mosheiov et al. [59].

Lemma 3.1. Let $\left(N, \sigma_{0}, p, a\right)$ be an $L E$ sequencing situation. Then for every $S \in \operatorname{con}\left(\sigma_{0}\right), C\left(\sigma_{S}, l_{S}^{\sigma_{S}}\right)=\min _{\sigma \in \Pi_{S}} C\left(\sigma, l_{S}^{\sigma}\right)$. 
Recall that $\sigma_{S}$ is the order which is attained from $\sigma_{0}$ by reordering the members in each $\sigma_{0}$-component of $S$ with respect to the SPT order, then we have the following theorem.

Theorem 3.2. Let $\left(N, \sigma_{0}, p, a\right)$ be an LE sequencing situation and $\left(N, v^{\lambda}\right)$ any corresponding $L E$ sequencing game. Then for any $S \subseteq N$,

$$
v^{\lambda}(S)=\sum_{i \in S} \Delta C\left(\sigma_{S}, i\right)+\lambda(S) \sum_{j \in N \backslash S} \Delta C\left(\sigma_{S}, j\right) .
$$

Proof. The result obviously holds for any connected coalition. Consider any non-connected coalition $S$ containing $k \sigma_{0}$-components, where $1<k<n$. Denote the $\sigma_{0}$-components of $S$ by $T_{1}, \ldots, T_{k}$ respectively. Now define $U_{h}=$ $\left\{i \in N \backslash S \mid l_{T_{h}}^{\sigma_{0}}<\sigma_{0}(i)<f_{T_{h+1}}^{\sigma_{0}}\right\}$ for any $1 \leq h \leq k-1$ and $U_{k}=\left\{i \in N \backslash S \mid l_{T_{k}}^{\sigma_{0}}<\right.$ $\left.\sigma_{0}(i)\right\}$. See Figure 3.1 for an illustration.

\begin{tabular}{|l|l|l|l|l|l|l|l|}
\hline$\cdots \cdots$ & $T_{1}$ & $U_{1}$ & $T_{2}$ & $U_{2}$ & $\cdots \cdots$ & $T_{k}$ & $U_{k}$ \\
\hline
\end{tabular}

FIGURE 3.1: The illustration of coalitions $T_{1}, \ldots, T_{k}$ and $U_{1}, \ldots, U_{k}$. 
Note that

$$
\begin{aligned}
& \sum_{i \in S} \Delta C\left(\sigma_{S}, i\right)+\lambda(S) \sum_{j \in N \backslash S} \Delta C\left(\sigma_{S}, j\right) \\
= & \sum_{h=1}^{k} \sum_{i \in T_{h}} \Delta C\left(\sigma_{T_{h}}, i\right)+\sum_{h=2}^{k} \sum_{m=h}^{k}\left[\left|T_{m}\right|\left(C\left(\sigma_{0}, l_{T_{h-1}}^{\sigma_{0}}\right)-C\left(\sigma_{T_{h-1}}, l_{T_{h-1}}^{\sigma_{T_{h-1}}}\right)\right)\right] \\
& +\lambda(S) \sum_{h=1}^{k} \sum_{m=h}^{k}\left[\left|U_{m}\right|\left(C\left(\sigma_{0}, l_{T_{h}}^{\sigma_{0}}\right)-C\left(\sigma_{T_{h}}, l_{T_{h}}^{\sigma_{T_{h}}}\right)\right)\right] \\
= & \sum_{h=1}^{k} \sum_{i \in T_{h}} \Delta C\left(\sigma_{T_{h}}, i\right) \\
& +\sum_{h=1}^{k-1}\left(\lambda(S)\left|U_{h}\right|+\sum_{m=h+1}^{k}\left(\lambda(S)\left|U_{m}\right|+\left|T_{m}\right|\right)\right)\left(C\left(\sigma_{0}, l_{T_{h}}^{\sigma_{0}}\right)-C\left(\sigma_{T_{h}}, l_{T_{h}}^{\sigma_{T_{h}}}\right)\right) \\
& +\lambda(S)\left|U_{k}\right|\left(C\left(\sigma_{0}, l_{T_{k}}^{\sigma_{0}}\right)-C\left(\sigma_{T_{k}}, l_{T_{k}}^{\sigma_{T_{k}}}\right)\right) \\
= & \max _{\sigma \in \Pi_{S}}\left\{\sum_{i \in S} \Delta C(\sigma, i)+\lambda(S) \sum_{j \in N \backslash S} \Delta C(\sigma, j)\right\} \\
= & v^{\lambda}(S),
\end{aligned}
$$

where the third equality holds because of Lemma 3.1 and the fact that SPT order is the optimal order for minimizing the total costs of players in a connected coalition.

It is also not hard to check that LE sequencing games $(N, v)$ are $\sigma_{0}$ component additive, and thus they are balanced. Moreover, for any core element $x \in C(N, v)$, we have for any $S \subseteq N, \sum_{i \in S} x_{i} \geq v(S) \geq v^{\lambda}(S)$. So we can conclude that LE sequencing game $\left(N, v^{\lambda}\right)$ is balanced for any $\lambda$.

Theorem 3.3. Let $\left(N, \sigma_{0}, p, a\right)$ be an $L E$ sequencing situation and $\left(N, v^{\lambda}\right)$ the corresponding cooperative game. Then $\left(N, v^{\lambda}\right)$ is balanced.

Proof. Note that for any share function $\lambda$, we must have that $v(N)=v^{\lambda}(N)$ and $v(S) \geq v^{\lambda}(S)$ for any $S \subset N$. So, it is sufficient to show that $(N, v)$ is balanced. Obviously, $(N, v)$ is supperadditive and for any $i \in N, v(i)=0$. We 
now just have to check that for any $S \subseteq N$

$$
v(S)=\sum_{T \in S / \sigma_{0}} v(T)
$$

Equation (3.3) clearly holds for any $S \in \operatorname{con}\left(\sigma_{0}\right)$. Given any non-connected coalition $S$, we use the same assumption about the coalition structure of $S$ as in the proof of Theorem 3.2. By observing the proof in Theorem 3.2, we can immediately obtain that

$$
\begin{aligned}
v(S)= & \sum_{h=1}^{k} \sum_{i \in T_{h}} \Delta C\left(\sigma_{T_{h}}, i\right) \\
& +\sum_{h=1}^{k-1}\left(\left|U_{h}\right|+\sum_{m=h+1}^{k}\left(\left|U_{m}\right|+\left|T_{m}\right|\right)\right)\left(C\left(\sigma_{0}, l_{T_{h}}^{\sigma_{0}}\right)-C\left(\sigma_{T_{h}}, l_{T_{h}}^{\sigma_{T_{h}}}\right)\right) \\
& +\left|U_{k}\right|\left(C\left(\sigma_{0}, l_{T_{k}}^{\sigma_{0}}\right)-C\left(\sigma_{T_{k}}, l_{T_{k}}^{\sigma_{T_{k}}}\right)\right) \\
= & \sum_{h=1}^{k}\left(\sum_{i \in T_{h}} \Delta C\left(\sigma_{T_{h}}, i\right)+\left(n-\sigma\left(l_{T_{h}}^{\sigma_{0}}\right)\right)\left(C\left(\sigma_{0}, l_{T_{h}}^{\sigma_{0}}\right)-C\left(\sigma_{T_{h}}, l_{T_{h}}^{\sigma_{T_{h}}}\right)\right)\right) \\
= & \sum_{h=1}^{k}\left(\sum_{i \in T_{h}} \Delta C\left(\sigma_{T_{h}}, i\right)+\sum_{j \in N \backslash T_{h}} \Delta C\left(\sigma_{T_{h}}, j\right)\right) \\
= & \sum_{T \in S / \sigma_{0}} v(T) .
\end{aligned}
$$

Then it follows that $(N, v)$ is a $\sigma_{0}$-component additive game and thus it is balanced. Take any core element $x$ of $C(N, v)$, we must have for any $S \subset N$,

$$
\sum_{i \in S} x_{i} \geq v(S) \geq v^{\lambda}(S)
$$

By noticing that $v(N)=v^{\lambda}(N)$, we can conclude that $\left(N, v^{\lambda}\right)$ is balanced.

Next, we show that the LE sequencing game $(N, v)$ is convex, the proof of which needs the following two lemmas. The first one provided by Borm et al. [9] shows a simple expression for the coefficients in the unique linear decomposition of a $\sigma_{0}$-component additive game into unanimity games. 
Lemma 3.4. [9] Let $(N, v)$ be a $\sigma_{0}$-component additive game and let $\sum_{S \subseteq N} \mu_{S} u_{S}$ be the linear decomposition of $(N, v)$ into unanimity games. Then, for every coalition $S \subseteq N$

$$
\mu_{S}= \begin{cases}v(S)-v\left(S \backslash\left\{f_{S}^{\sigma_{0}}\right\}\right)-v\left(S \backslash\left\{l_{S}^{\sigma_{0}}\right\}\right)+v\left(S \backslash\left\{f_{S}^{\sigma_{0}}, l_{S}^{\sigma_{0}}\right\}\right) & \text { if } S \in \operatorname{con}\left(\sigma_{0}\right), \\ 0 & \text { otherwise. }\end{cases}
$$

By using the above lemma, we can also give an expression for the value of these coefficients for an LE sequencing game $(N, v)$ since it is $\sigma_{0}$-component additive.

Lemma 3.5. Let $\left(N, \sigma_{0}, p, a\right)$ be an LE sequencing situation. Let $(N, v)$ be the corresponding $L E$ sequencing game and $\sum_{S \subseteq N} \mu_{S} u_{S}$ be the linear decomposition of $(N, v)$ into unanimity games. Then, for every $S \in \operatorname{con}\left(\sigma_{0}\right)$

$$
\mu_{S}= \begin{cases}\sum_{\sigma_{S}\left(l_{S}^{\sigma_{0}}\right) \leq r<\sigma_{S}\left(f_{S}^{\sigma_{0}}\right)} \theta(r)\left(p_{\sigma_{S}^{-1}(r+1)}-p_{\sigma_{S}^{-1}(r)}\right) & \text { if } \sigma_{S}\left(l_{S}^{\sigma_{0}}\right)<\sigma_{S}\left(f_{S}^{\sigma_{0}}\right), \\ 0 & \text { otherwise. }\end{cases}
$$

Proof. Let $S \subseteq N$ be a connected coalition with respect to $\sigma_{0}$. For simplicity, let us denote $f_{S}^{\sigma_{0}}$ by $i, l_{S}^{\sigma_{0}}$ by $j, S \backslash\{i\}$ by $S_{1}, S \backslash\{j\}$ by $S_{2}, S \backslash\{i, j\}$ by $S_{3}$ and denote $I^{S_{1}}$ by $I_{1}, I^{S_{3}}$ by $I_{3}$. It is easy to see that there must be an feasible permutation process $\rho_{3}=\left\{\tau_{1}, \ldots, \tau_{I_{3}}\right\} \in \mathscr{P}\left(\sigma_{0}, \sigma_{S_{3}}\right)$ for $S_{3}$, which starting with $\sigma_{0}$ and ending with $\sigma_{S_{3}}$. Once the order $\sigma_{S_{3}}$ is reached by $\rho_{3}, \sigma_{S_{1}}$ can be obtained by successive switches between $j$ and the players in front of him. So, there must be a feasible permutation process $\rho_{1} \in \mathscr{P}\left(\sigma_{0}, \sigma_{S_{1}}\right)$ for $S_{1}$ and $\rho_{1}=\left\{\tau_{1}, \ldots, \tau_{I_{3}}, \tau_{I_{3}+1}, \ldots, \tau_{I_{1}}\right\}$. Similarly, once the order $\sigma_{S_{1}}$ is reached by $\rho_{1}, \sigma_{S}$ can be obtained by successive switches between $i$ and the players behind him. So, there must be an feasible permutation process $\rho \in$ $\mathscr{P}\left(\sigma_{0}, \sigma_{S}\right)$ for $S$ and $\rho=\left\{\tau_{1}, \ldots, \tau_{I_{3}}, \tau_{I_{3}+1}, \ldots, \tau_{I_{1}}, \tau_{I_{1}+1}, \ldots, \tau_{I^{S}}\right\}$.

According to Lemma 3.1, we have

$$
v(S)=\sum_{k=1}^{I^{S}} g\left(\tau_{k}, \sigma_{k-1}, i_{k}, j_{k}\right)
$$


Similarly,

$$
v\left(S_{1}\right)=\sum_{k=1}^{I_{1}} g\left(\tau_{k}, \sigma_{k-1}, i_{k}, j_{k}\right) .
$$

Hence,

$$
v(S)-v\left(S_{1}\right)=\sum_{k=I_{1}+1}^{I^{S}} g\left(\tau_{k}, \sigma_{k-1}, i_{k}, j_{k}\right) .
$$

From the definition of $g(\tau, \sigma, i, j)$, it is not hard to see that equation (3.4) can be rewritten as

$$
v(S)-v\left(S_{1}\right)=\sum_{\sigma_{0}(i) \leq r<\sigma_{S}(i)} \theta(r)\left(p_{i}-p_{\sigma_{S}^{-1}(r)}\right) .
$$

We also notice that when $\sigma_{S_{3}}$ is reached by $\rho_{3}, \sigma_{S_{2}}$ can be obtained by successive switches between $i$ and the players behind him. We denote the number of the adjacent exchanges between $i$ and the players behind him by $m,{ }^{1}$ and these adjacent exchanges are denoted by $\tau_{1}^{\prime}, \ldots, \tau_{m}^{\prime}$. Then there must be an feasible permutation process $\rho_{2} \in \mathscr{P}\left(\sigma_{0}, \sigma_{S_{2}}\right)$ for $S_{2}$ and $\rho_{2}=$ $\left\{\tau_{1}, \ldots, \tau_{I_{3}}, \tau_{1}^{\prime}, \ldots, \tau_{m}^{\prime}\right\}$. It holds that

$$
v\left(S_{2}\right)=\sum_{k=1}^{I_{3}} g\left(\tau_{k}, \sigma_{k-1}, i_{k}, j_{k}\right)+\sum_{k=1}^{m} g\left(\tau_{k}^{\prime}, \sigma_{k-1}^{\prime}, i_{k}^{\prime}, j_{k}^{\prime}\right),
$$

where $\sigma_{0}^{\prime}=\sigma_{S_{3}}$. Since

$$
v\left(S_{3}\right)=\sum_{k=1}^{I_{3}} g\left(\tau_{k}, \sigma_{k-1}, i_{k}, j_{k}\right),
$$

then we have

$$
v\left(S_{2}\right)-v\left(S_{3}\right)=\sum_{k=1}^{m} g\left(\tau_{k}^{\prime}, \sigma_{k-1}^{\prime}, i_{k}^{\prime}, j_{k}^{\prime}\right) .
$$

\footnotetext{
${ }^{1}$ In fact, $m$ equals to the number of inverted pairs composed by $i$ and the players in $S_{3}$.
} 
Suppose that $\sigma_{S}(j) \geq \sigma_{S}(i)$. Then equation (3.5) can be rewritten as

$$
v\left(S_{2}\right)-v\left(S_{3}\right)=\sum_{\sigma_{0}(i) \leq r<\sigma_{S}(i)} \theta(r)\left(p_{i}-p_{\sigma_{S}^{-1}(r)}\right) .
$$

Hence, from Lemma 3.4, we have

$$
\mu_{S}=v(S)-v\left(S_{1}\right)-\left(v\left(S_{2}\right)-v\left(S_{3}\right)\right)=0 .
$$

Suppose that $\sigma_{S}(j)<\sigma_{S}(i)$. Different from the above case, equation (3.5) is rewritten as

$$
v\left(S_{2}\right)-v\left(S_{3}\right)=\sum_{\sigma_{0}(i) \leq r<\sigma_{S}(j)} \theta(r)\left(p_{i}-p_{\sigma_{S}^{-1}(r)}\right)+\sum_{\sigma_{S}(j) \leq r<\sigma_{S}(i)} \theta(r)\left(p_{i}-p_{\sigma_{S}^{-1}(r+1)}\right) .
$$

Hence, by using Lemma 3.4, we have

$$
\mu_{S}=v(S)-v\left(S_{1}\right)-\left(v\left(S_{2}\right)-v\left(S_{3}\right)\right)=\sum_{\sigma_{S}(j) \leq r<\sigma_{S}(i)} \theta(r)\left(p_{\sigma_{S}^{-1}(r+1)}-p_{\sigma_{S}^{-1}(r)}\right) .
$$

In fact, these coefficients can be proved to be nonnegative. Since unanimity games are convex, we therefore obtain that also $(N, v)$ is convex.

Theorem 3.6. Let $\left(N, \sigma_{0}, p, a\right)$ be an LE sequencing situation. Then the corresponding $L E$ sequencing game $(N, v)$ is convex.

Proof. Lemma 3.5 implies that the coefficient of a connected coalition $S$ is independent of any permutation process for $S$, which means that as well as all connected coalitions of $N$ were derived, the corresponding coefficients could be obtained. Thus we can conclude that the LE sequencing game $(N, v)$ is a nonnegative combination of unanimity games. Since unanimity games are convex, we can obtain the convexity of the LE sequencing game $(N, v)$. 


\subsection{The family of LE-EGS rules and the $\Gamma$-rule}

Motivated by the fact that core allocations for $(N, v)$ are also core allocations for $\left(N, v^{\lambda}\right)$ for any $\lambda$, we focus on LE sequencing games $(N, v)$. This is the setting where the worth of a coalition $S$ includes all additional benefits that their cooperative behavior generates. In that sense, the constraints that define the core of $(N, v)$ are tightest, and this version of the game could be considered as the "hardest". One could also consider variations of the game $\left(N, v^{\lambda}\right)$ where an additional player (a system designer) obtains the taxes implied by $\lambda$, but this would go beyond the scope of this note.

\subsubsection{The family of LE-EGS rules}

We now introduce the LE-EGS family, which is inspired EGS rule introduced and characterized in [21]. The idea of the EGS rule is to divide the cost savings obtained by an adjacent exchange equally between the two involved players. Here, we adopt this idea and define the $L E-E G S^{P e}$ rule as follows. Let $P e=\left\{\tau_{1}, \ldots, \tau_{I^{N}}\right\} \in \mathscr{P}\left(\sigma_{0}, \sigma_{N}\right)$ be a feasible permutation process so that $\operatorname{Pe}\left(\sigma_{0}\right)=\sigma_{N}, \sigma_{k}=\tau_{k}\left(\sigma_{k-1}\right)$ for any $1 \leq k \leq I^{N}$, and let $i_{k}=\sigma_{k-1}^{-1}\left(\tau_{k}\right)$, $j_{k}=\sigma_{k-1}^{-1}\left(\tau_{k}+1\right)$ be the two players involved in the $k$-th adjacent exchange. Then let the allocation to player $i \in N$ be

$$
\operatorname{LE}_{-E G S_{i}^{P e}}\left(N, \sigma_{0}, p, a\right)=\frac{1}{2} \sum_{k=1}^{I^{N}} g\left(\tau_{k}, \sigma_{k-1}, i_{k}, j_{k}\right) \delta_{i}\left(i_{k}, j_{k}\right),
$$

where $\delta_{i}$ is simply an indicator variable to collect the payments made to player $i$,

$$
\delta_{i}\left(i_{k}, j_{k}\right)= \begin{cases}1 & \text { if } i_{k}=i \text { or } j_{k}=i \\ 0 & \text { otherwise }\end{cases}
$$

The family of all LE-EGS rules is defined by

$$
\operatorname{LEF}\left(N, \sigma_{0}, p, a\right)=\left\{L E-E G S^{P e}\left(N, \sigma_{0}, p, a\right) \mid P e \in \mathscr{P}\left(\sigma_{0}, \sigma_{N}\right)\right\} .
$$

At this point, it is interesting to know whether any member of this family yields a core allocation. The following example shows that the answer is 
negative.

Example 3.2. Let $\left(N, \sigma_{0}, p, a\right)$ be an LE sequencing situation, where $N=\{1,2,3\}$, $\sigma_{0}=(1,2,3), p=\left\{3,2, \frac{9}{5}\right\}$ and $a=-1$. Then it can be observed that $\sigma_{N}=$ $(3,2,1), I^{N}=3$ and there are two feasible permutation processes $P e_{1}=\left\{\tau_{1}, \tau_{2}, \tau_{3}\right\}$, $P e_{2}=\left\{\tau_{1}^{\prime}, \tau_{2}^{\prime}, \tau_{3}^{\prime}\right\}$ which are illustrated in Figure 3.2.

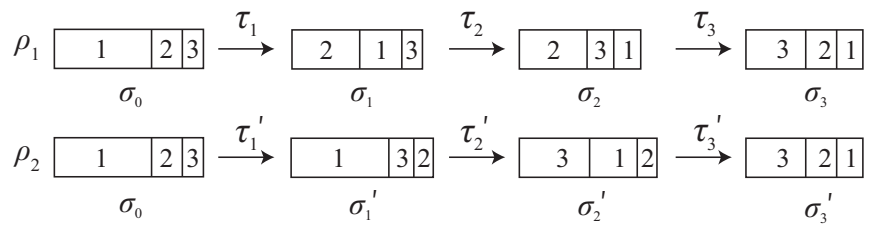

FIgURE 3.2: The feasible permutation processes $P e_{1}$ and $P e_{2}$

We can easily compute that LE-EGS ${ }^{P e_{1}}=\left(\frac{7}{5}, \frac{6}{5}, \frac{3}{5}\right)$ and $L E-E G S^{P e_{2}}=\left(\frac{23}{15}, \frac{2}{5}, \frac{19}{15}\right)$. The worths of the corresponding $L E$ sequencing game $(N, v)$ are displayed in $T a-$ ble 3.1 .

TABLE 3.1 Worths of the LE sequencing game $(N, v)$

\begin{tabular}{cccccccc}
\hline$S$ & $\{1\}$ & $\{2\}$ & $\{3\}$ & $\{1,2\}$ & $\{1,3\}$ & $\{2,3\}$ & $\{1,2,3\}$ \\
\hline$v(S)$ & 0 & 0 & 0 & 2 & 0 & $\frac{2}{15}$ & $\frac{16}{5}$ \\
\hline
\end{tabular}

We can see that $L E-E G S^{P e_{1}} \in C(N, v)$, but $L E-E G S^{P e_{2}} \notin C(N, v)$.

\subsubsection{The $\Gamma$-rule}

We now introduce a particular feasible permutation process $P e \in \mathscr{P}\left(\sigma_{0}, \sigma_{N}\right)$ which actually yields a core allocation. It is defined by the following sequence of adjacent exchanges:

Step 1 . Set $k=1$.

Step 2. Find the earliest two neighboring jobs $i_{k}, j_{k}$ that are inverted in order $\sigma_{k-1}$. If no such pair exists, then stop (and $\sigma_{k-1}=\sigma_{N}$ ). Formally,

$$
i_{k}:=\arg \min \left\{\sigma_{k-1}(\ell) \mid p_{\ell}>p_{j}, \sigma_{k-1}(j)=\sigma_{k-1}(\ell)+1\right\} .
$$


Step 3. While $\left(i_{k}, j_{k}\right)$ is an inverted pair in $\sigma_{k-1}$ :

- Exchange $i_{k}$ with $j_{k}$ to obtain $\sigma_{k}$ from $\sigma_{k-1}$, add the adjacent exchange $\tau_{k}$ with $\tau_{k}\left(\sigma_{k-1}\right)=\sigma_{k}$ to $P e$

- Let $k=k+1$

- Let $i_{k}:=i_{k-1}$, let $j_{k}:=\sigma_{k-1}\left(i_{k}\right)+1$ be the next job after $i_{k}$ (break while if $\sigma_{k-1}\left(i_{k}\right)=n$ )

Step 4. Goto Step 2

The above sequence of adjacent exchanges is denoted by $\Gamma$. It is obvious that the so-computed permutation process $P e$ is feasible. For notational convenience, let us call the EGS rule that is based on this permutation process $P e$ the $\Gamma$-rule. We define for all $i \in N$

$$
\Gamma_{i}\left(N, \sigma_{0}, p, a\right)=L E-E G S_{i}^{P e}\left(N, \sigma_{0}, p, a\right),
$$

with $P e$ being the permutation process computed as above. In order to show that the $\Gamma$-rule of any LE sequencing situation yields a core element for the corresponding LE sequencing game, we derive the following lemmas.

The first lemma gives a new expression of $v(S)$ for any $S \in \operatorname{con}\left(\sigma_{0}\right)$, which is the sum of the gains of adjacent exchanges in a feasible permutation process for $S$.

Lemma 3.7. Let $\left(N, \sigma_{0}, p, a\right)$ be an LE sequencing situation and $(N, v)$ the corresponding $L E$ sequencing game. For any $S \in \operatorname{con}\left(\sigma_{0}\right)$ and any feasible permutation process $\mathrm{Pe} \in \mathscr{P}\left(\sigma_{0}, \sigma_{S}\right)$,

$$
v(S)=\sum_{k=1}^{I^{S}} g\left(\tau_{k}, \sigma_{k-1}, i_{k}, j_{k}\right) .
$$

Proof. From Theorem 3.2, we have that for any $S \subseteq N$,

$$
v(S)=\max _{\sigma \in \Pi_{S}} \sum_{i \in N} \Delta C(\sigma, i)=\sum_{i \in N} \Delta C\left(\sigma_{S}, i\right) .
$$


Since $P e=\left\{\tau_{1}, \tau_{2}, \ldots, \tau_{I^{s}}\right\}$ is feasible, we have $\sigma_{I^{s}}=\sigma_{S}$. Hence,

$$
\begin{aligned}
\sum_{i \in N} \Delta C\left(\sigma_{S}, i\right) & =\sum_{i \in N}\left[C\left(\sigma_{0}, i\right)-C\left(\sigma_{S}, i\right)\right] \\
& =\sum_{i \in N} \sum_{k=1}^{I^{S}}\left(C\left(\sigma_{k-1}, i\right)-C\left(\sigma_{k}, i\right)\right) \\
& =\sum_{k=1}^{I^{S}} \sum_{i \in N}\left(C\left(\sigma_{k-1}, i\right)-C\left(\sigma_{k}, i\right)\right) \\
& =\sum_{k=1}^{I^{S}} g\left(\tau_{k}, \sigma_{k-1}, i_{k}, j_{k}\right) .
\end{aligned}
$$

The second lemma shows that in a feasible permutation process for $N$, the total gains of players in $S$ are not less than $v(S)$.

Lemma 3.8. Let $\left(N, \sigma_{0}, p, a\right)$ be an $L E$ sequencing situation and $(N, v)$ the corresponding $L E$ sequencing game. If $P e \in \mathscr{P}\left(\sigma_{0}, \sigma_{N}\right)$ is a feasible permutation procedure computed by $\Gamma$, then for any $S \in \operatorname{con}\left(\sigma_{0}\right)$

$$
\sum_{\substack{k=1, \cdots, I^{N} \\ i_{k}, j_{k} \in S}} g\left(\tau_{k}, \sigma_{k-1}, i_{k}, j_{k}\right) \geq v(S) .
$$

Proof. Let $P e^{\prime}=\left\{\tau_{1}^{\prime}, \ldots, \tau_{I^{S}}^{\prime}\right\}$ be a feasible permutation process by adopting procedure $\Gamma$ for coalition $S$. Let $\tau_{k}^{\prime}\left(\sigma_{k-1}^{\prime}\right)=\sigma_{k}^{\prime}$ for any $1 \leq k \leq I^{S}$ and $\sigma_{0}=$ $\sigma_{0}^{\prime}$. For every $\tau_{k}^{\prime} \in P e^{\prime}$, the associated exchange players are denoted $i_{k}^{\prime}$ and $j_{k}^{\prime}$. Then according to Lemma 3.7, we have

$$
v(S)=\sum_{k=1}^{I^{S}} g\left(\tau_{k}^{\prime}, \sigma_{k-1}^{\prime}, i_{k}^{\prime}, j_{k}^{\prime}\right) .
$$


So, we are done if we can show that

$$
\sum_{\substack{k=1, \cdots, I^{N} \\ i_{k}, j_{k} \in S}} g\left(\tau_{k}, \sigma_{k-1}, i_{k}, j_{k}\right) \geq \sum_{k=1}^{I^{S}} g\left(\tau_{k}^{\prime}, \sigma_{k-1}^{\prime}, i_{k}^{\prime}, j_{k}^{\prime}\right) .
$$

Intuitively, this inequality should hold: first, every fixed inverted pair $i, j \in S$ appears exactly once in each of the two sums. Second, since at the moment that we do an adjacent exchange of a fixed inverted pair $i, j \in S$, due to the procedure $\Gamma$, the position at which that exchange happens in $P e$ can only be earlier than in $P e^{\prime}$. Remembering (3.1), this yields the claim.

More formally, let $i, j$ be a fixed inverted pair of jobs in $S$. Note that $p_{i}>$ $p_{j}$. Let $k$ be the iteration in $P e$ so that $i_{k}=i, j_{k}=j$, and let $k^{\prime}$ be the corresponding iteration in $P e^{\prime}$ so that $i_{k^{\prime}}=i, j_{k^{\prime}}=j$. Define sets $U^{\prime}, M^{\prime}, D^{\prime}$ and $U$ as follows

$$
\begin{gathered}
U^{\prime}=P\left(\sigma_{0}, i\right) \cap\left\{z \in S \mid p_{z}>p_{i}\right\}, \\
M^{\prime}=P\left(\sigma_{0}, j\right) \cap F\left(\sigma_{0}, i\right) \cap\left\{z \in S \mid p_{z} \geq p_{i}\right\}, \\
D^{\prime}=P\left(\sigma_{0}, j\right) \cap F\left(\sigma_{0}, i\right) \cap\left\{z \in S \mid p_{z}<p_{i}\right\}, \\
U=P\left(\sigma_{0}, i\right) \cap\left\{z \in N \mid p_{z}>p_{i}\right\} .
\end{gathered}
$$

Then by definition of procedure $\Gamma$, in $\sigma_{k^{\prime}-1}^{\prime}$, all jobs in $U^{\prime} \cup M^{\prime}$ must be in positions later than $j$. Moreover, all jobs in $D^{\prime}$ must be in positions before $i$. Hence, we conclude that

$$
\sigma_{k^{\prime}-1}^{\prime}(i)=\sigma_{0}(i)+\left|D^{\prime}\right|-\left|U^{\prime}\right|
$$

Likewise, we have that

$$
\sigma_{k-1}(i)=\sigma_{0}(i)+\left|D^{\prime}\right|-|U|
$$

Since $U^{\prime} \subseteq U$, it follows that $\sigma_{k-1}(i) \leq \sigma_{k^{\prime}-1}^{\prime}(i)$. Recalling (3.1), we conclude

$$
g\left(\tau_{k}, \sigma_{k-1}, i, j\right) \geq g\left(\tau_{k^{\prime}}^{\prime}, \sigma_{k^{\prime}-1}^{\prime}, i, j\right) .
$$


Inequality (3.6) now follows, since each inverted pair $i, j \in S$ appears exactly once in both of the sums of (3.6).

As promised, we now show that the $\Gamma$-rule yields a core element for any LE sequencing game $(N, v)$.

Theorem 3.9. Let $\left(N, \sigma_{0}, p, a\right)$ be an $L E$ sequencing situation and $(N, v)$ the corresponding $L E$ sequencing game. Then the $\Gamma$-rule gives a core element of $(N, v)$.

Proof. Let again $P e=\left\{\tau_{1}, \tau_{2}, \ldots, \tau_{I^{N}}\right\}$ be the permutation process derived from $\Gamma$. Note that

$$
\begin{aligned}
\sum_{i \in N} \Gamma_{i}\left(N, \sigma_{0}, p, a\right) & =\sum_{i \in N} \frac{1}{2} \sum_{k=1}^{I^{N}} g\left(\tau_{k}, \sigma_{k-1}, i_{k}, j_{k}\right) \delta_{i}\left(i_{k}, j_{k}\right) \\
& =\sum_{k=1}^{I^{N}} g\left(\tau_{k}, \sigma_{k-1}, i_{k}, j_{k}\right) \\
& =v(N),
\end{aligned}
$$

where the third equality follows from Lemma 3.7. For any $S \in \operatorname{con}\left(\sigma_{0}\right)$,

$$
\begin{aligned}
& \sum_{i \in S} \Gamma_{i}\left(N, \sigma_{0}, p, a\right)=\sum_{i \in S} \frac{1}{2} \sum_{k=1}^{I^{N}} g\left(\tau_{k}, \sigma_{k-1}, i_{k}, j_{k}\right) \delta_{i}\left(i_{k}, j_{k}\right) \\
& \geq \sum_{i \in S} \frac{1}{2} \sum_{\substack{k=1 \cdots I^{N} \\
i_{k}, j_{k} \in S}} g\left(\tau_{k}, \sigma_{k-1}, i_{k}, j_{k}\right) \delta_{i}\left(i_{k}, j_{k}\right) \\
& =\sum_{\substack{k=1 \cdots I^{N} \\
i_{k}, j_{k} \in S}} g\left(\tau_{k}, \sigma_{k-1}, i_{k}, j_{k}\right) \\
& \geq v(S),
\end{aligned}
$$

the last inequality following from Lemma 3.8. From $\sigma_{0}$-component additivity, we finally conclude $\Gamma\left(N, \sigma_{0}, p, a\right) \in C(N, v)$. 


\subsubsection{A characterization of the $\Gamma$-rule}

Generally spoken, an allocation rule $\phi$ assigns to each LE sequencing game an allocation $x \in \mathbb{R}^{n}$. We now aim at characterizing the $\Gamma$-rule in terms of (more or less) natural properties imposed on $\phi$. To that end, for given $\left(N, \sigma_{0}, p, a\right)$, denote the set of successors of a player's job $i$ with a smaller nominal processing time than that of player $i$, by $D\left(\sigma_{0}, i\right)$, i.e., $D\left(\sigma_{0}, i\right):=F\left(\sigma_{0}, i\right) \cap\left\{j \mid p_{j}<\right.$ $\left.p_{i}\right\}$. Player $i$ is a $D$-equivalence player with respect to LE sequencing situations $\left(N, \sigma_{0}, p, a\right),\left(N, \sigma_{1}, p, a\right)$ if $F\left(\sigma_{0}, i\right)=F\left(\sigma_{1}, i\right)$, and for any $k, l \in$ $D\left(\sigma_{0}, i\right), \sigma_{0}(k)<\sigma_{0}(l)$ implies $\sigma_{1}(k)<\sigma_{1}(l)$. We say that an inverted pair $(i, j)$ is a head pair with respect to $\left(N, \sigma_{0}, p, a\right)$ if $\sigma_{0}(i)=\sigma_{0}(j)-1$ and for any $k \in P\left(\sigma_{0}, i\right), p_{k} \leq p_{i}$. Consider the following properties.

- Efficiency: Let $\left(N, \sigma_{0}, p, a\right)$ be an LE sequencing situation. Then we have $\sum_{i \in N} \phi_{i}\left(N, \sigma_{0}, p, a\right)=\sum_{i \in N} \Delta C\left(\sigma_{N}, i\right)$.

- Dummy: Let $\left(N, \sigma_{0}, p, a\right)$ be an LE sequencing situation. If $P\left(\sigma_{0}, i\right)=$ $P\left(\sigma_{N}, i\right)$ for some $i \in N$, then $\phi_{i}\left(N, \sigma_{0}, p, a\right)=0$.

- $D$-equivalence: Let $\left(N, \sigma_{0}, p, a\right),\left(N, \sigma_{1}, p, a\right)$ be two LE sequencing situations. If some player $i \in N$ is a $D$-equivalence player with respect to these two LE sequencing situations, then $\phi_{i}\left(N, \sigma_{0}, p, a\right)=\phi_{i}\left(N, \sigma_{1}, p, a\right)$.

- Head switch property: Let $\left(N, \sigma_{0}, p, a\right),\left(N, \sigma_{1}, p, a\right)$ be two LE sequencing situations and $i, j \in N$ be such that $\sigma_{0}(i)=\sigma_{0}(j)-1, \sigma_{1}(i)=\sigma_{0}(j)$, $\sigma_{1}(j)=\sigma_{0}(i)$ and $\sigma_{1}(k)=\sigma_{0}(k)$ for any $k \in N \backslash\{i, j\}$. If $(i, j)$ is a head pair with respect to $\left(N, \sigma_{0}, p, a\right)$, then

$$
\phi_{i}\left(N, \sigma_{1}, p, a\right)-\phi_{i}\left(N, \sigma_{0}, p, a\right)=\phi_{j}\left(N, \sigma_{1}, p, a\right)-\phi_{j}\left(N, \sigma_{0}, p, a\right) .
$$

The last two properties are the variations of equivalence and switch property introduced by Curiel et al. [23] for characterizing the EGS rule. In the next theorem, we show that the $\Gamma$-rule satisfies efficiency, the dummy property, $D$-equivalence, and the head switch property. Before that, we will give below another equivalent formulation of the $\Gamma$-rule, which will be very useful in later proofs. 
Let $\left(N, \sigma_{0}, p, a\right)$ be an LE sequencing situation. Define $g_{i j}^{r}$ as the cost savings of the grand coalition generated from the switch of two neighboring players $i, j$ with $\sigma_{0}(i)=\sigma_{0}(j)-1$ and $\sigma_{0}(i)=r$. For any player $i \in N$, denote $U\left(\sigma_{0}, i\right)=P\left(\sigma_{0}, i\right) \cap\left\{k \mid p_{k}>p_{i}\right\}$, which represents the set of predecessors of $i$ who have larger nominal processing times.

Let $U\left(\sigma_{0}, i\right)=\left\{u_{1}, \ldots, u_{h_{1}}\right\}$ with $p_{u_{1}} \geq \ldots \geq p_{u_{h_{1}}}$ and $D\left(\sigma_{0}, i\right)=\left\{d_{1}, \ldots, d_{h_{2}}\right\}$ with $\sigma_{0}\left(d_{1}\right)<\ldots<\sigma_{0}\left(d_{h_{2}}\right)$. By observing the steps of the procedure $\Gamma$ and the definition of the LE-EGS rule, the $\Gamma$-rule can be rewritten as the following way:

$$
\Gamma_{i}\left(N, \sigma_{0}, p, a\right)=\frac{1}{2}\left(\sum_{m=1}^{h_{1}} g_{u_{m} i}^{r-m}+\sum_{m=1}^{h_{2}} g_{i d_{m}}^{r-h_{1}+m-1}\right),
$$

for all $i \in N$. To see why, recall how $\Gamma$ is defined, and observe that for any $1 \leq$ $m \leq h_{1}$, jobs $u_{m}$ and $i$ can not become adjacent before $u_{1}, \ldots, u_{m-1}$ have moved to positions behind $i$. Hence, $u_{m}$ is exactly in position $(r-m)$ when the adjacent exchange of $u_{m}$ and $i$ takes place. This continues until $u_{h_{1}}$ is on a position somewhere behind $i$. Consequently, $i$ will be in positions $r-h_{1}$, $r-h_{1}+1, \ldots, r-h_{1}+h_{2}-1$ when the adjacent exchanges with $d_{1}, d_{2}, \ldots, d_{h_{2}}$ take place. After $i$ and $d_{h_{2}}$ have exchanged their positions, $i$ will remain at its position. Observe that the order in which jobs $U\left(\sigma_{0}, i\right)$ exchange with $i$, only depends on the (nominal) sizes of $u_{1}, \ldots, u_{h_{1}}$, while the order in which $i$ exchanges with jobs $D\left(\sigma_{0}, i\right)$, only depends on the order of $d_{1}, \ldots, d_{h_{2}}$ in $\sigma_{0}$.

Theorem 3.10. The $\Gamma$-rule is the unique allocation rule for LE sequencing situations that satisfies efficiency, the dummy property, D-equivalence and the head switch property.

Proof. The fact that the $\Gamma$-rule satisfies efficiency and dummy properties is obvious. We only have to show that the $\Gamma$-rule satisfies the remaining two properties. Let $\left(N, \sigma_{0}, p, a\right)$ be an LE sequencing situation. Given any $i \in$ $N$, let $\left(N, \sigma_{1}, p, a\right)$ be an LE sequencing situation satisfying that $F\left(\sigma_{0}, i\right)=$ $F\left(\sigma_{1}, i\right)$ and for any $k, l \in D\left(\sigma_{0}, i\right), \sigma_{0}(k)<\sigma_{0}(l)$ implies $\sigma_{1}(k)<\sigma_{1}(l)$. Let $D(\sigma, i)=\left\{d_{1}, \ldots, d_{h_{2}}\right\}$ with $\sigma_{0}\left(d_{1}\right)<\ldots<\sigma_{0}\left(d_{h_{2}}\right)$. Then we must have that $\sigma_{1}\left(d_{1}\right)<\ldots<\sigma_{1}\left(d_{h_{2}}\right)$. By (3.7), we can clearly see that $\Gamma_{i}\left(N, \sigma_{0}, p, a\right)=$ $\Gamma_{i}\left(N, \sigma_{1}, p, a\right)$. Thus, the $\Gamma$-rule possesses $D$-equivalence property. 
Let $\left(N, \sigma_{0}, p, a\right)$ be an LE sequencing situation and $(i, j)$ the head pair with respect to $\left(N, \sigma_{0}, p, a\right)$. Let $\left(N, \sigma_{1}, p, a\right)$ be an LE sequencing situation satisfying that $\sigma_{1}(i)=\sigma_{0}(j), \sigma_{1}(j)=\sigma_{0}(i)$ and $\sigma_{1}(k)=\sigma_{0}(k)$ for any $k \in N \backslash\{i, j\}$. Let $\left(d_{1}, \ldots, d_{h_{2}}\right)$ and $\left(d_{1}^{\prime}, \ldots, d_{h_{2}}^{\prime}\right)$ be two orderings of $D\left(\sigma_{0}, i\right)$ with $\sigma_{0}\left(d_{1}\right)<\cdots<\sigma_{0}\left(d_{h_{2}}\right)$ and $p_{d_{1}^{\prime}} \leq \cdots \leq p_{d_{h_{2}}^{\prime}}$ respectively, and $U\left(\sigma_{0}, j\right)=$ $\left\{u_{1}^{\prime}, \ldots, u_{h_{1}^{\prime}}^{\prime}\right\}$ with $p_{u_{1}^{\prime}} \geq \ldots \geq p_{u_{h_{1}^{\prime}}^{\prime}}$. Assume that $i$ is in position $r$ with respect to $\sigma_{0}$. For player $i$, by noticing that $(i, j)$ is a head pair and $d_{1}=j$, we can get from (3.7) that

$$
\Gamma_{i}\left(N, \sigma_{0}, p, a\right)=\frac{1}{2}\left(g_{i j}^{r}+\sum_{m=2}^{h_{2}} g_{i d_{m}}^{r+m-1}\right),
$$

and

$$
\Gamma_{i}\left(N, \sigma_{1}, p, a\right)=\frac{1}{2} \sum_{m=2}^{h_{2}} g_{i d_{m}}^{r+m-1}
$$

Thus,

$$
\Gamma_{i}\left(N, \sigma_{1}, p, a\right)-\Gamma_{i}\left(N, \sigma_{0}, p, a\right)=-\frac{1}{2} g_{i j}^{r} .
$$

For player $j$, by noticing that $u_{1}^{\prime}=i$, we can get from (3.7) that

$$
\Gamma_{j}\left(N, \sigma_{0}, p, a\right)=\frac{1}{2}\left(g_{i j}^{r}+\sum_{m=2}^{h_{1}^{\prime}} g_{u_{m}^{\prime} j}^{r+1-m}+\sum_{m=1}^{h_{2}^{\prime}} g_{j d_{m}^{\prime}}^{r-h_{1}^{\prime}+m}\right),
$$

and

$$
\Gamma_{j}\left(N, \sigma_{1}, p, a\right)=\frac{1}{2}\left(\sum_{m=2}^{h_{1}^{\prime}} g_{u_{m}^{\prime} j}^{r+1-m}+\sum_{m=1}^{h_{2}^{\prime}} g_{j d_{m}^{\prime}}^{r-h_{1}^{\prime}+m}\right) .
$$

Then

$$
\Gamma_{j}\left(N, \sigma_{1}, p, a\right)-\Gamma_{j}\left(N, \sigma_{0}, p, a\right)=-\frac{1}{2} g_{i j}^{r}=\Gamma_{i}\left(N, \sigma_{1}, p, a\right)-\Gamma_{i}\left(N, \sigma_{0}, p, a\right) .
$$

Thus, the $\Gamma$-rule possesses head switch property.

It remains to prove the uniqueness part. Suppose that $\phi$ is an allocation rule with the four mentioned properties. For any LE sequencing situation 
$(N, \sigma, p, a)$, define $M_{\sigma}=\left\{(k, l) \mid \sigma(k)=\sigma(l)-1\right.$ and $\left.p_{k}>p_{l}\right\}$. We will prove this part by induction on the cardinality of $M_{\sigma}$. If $\left|M_{\sigma}\right|=0$, the dummy property implies that $\Gamma_{i}(N, \sigma, p, a)=\phi_{i}(N, \sigma, p, a)=0$ for all $i \in N$. Suppose that for any $\left|M_{\sigma}\right| \leq m, \Gamma(N, \sigma, p, a)=\phi(N, \sigma, p, a)$. Let $\left(N, \sigma_{0}, p, a\right)$ be an LE sequencing situation with $\left|M_{\sigma_{0}}\right|=m+1$ and $(i, j)$ the head pair with respect to $\left(N, \sigma_{0}, p, a\right)$. Let $\left(N, \sigma_{1}, p, a\right)$ be an LE sequencing situation such that $\sigma_{0}(i)=$ $\sigma_{1}(j), \sigma_{0}(j)=\sigma_{1}(i)$ and $\sigma_{0}(k)=\sigma_{1}(k)$ for any $k \in N \backslash\{i, j\}$. Thus, $\left|M_{\sigma_{1}}\right|=$ $m$. From the $D$-equivalence property and the induction assumption it follows that for any $k \in N \backslash\{i, j\}$

$$
\phi_{k}\left(N, \sigma_{0}, p, a\right)=\phi_{k}\left(N, \sigma_{1}, p, a\right)=\Gamma_{k}\left(N, \sigma_{1}, p, a\right)=\Gamma_{k}\left(N, \sigma_{0}, p, a\right) .
$$

Suppose that $i$ is in position $r$ with respect to $\sigma_{0}$. From the efficiency and head switch properties we must have

$$
\begin{aligned}
& \phi_{i}\left(N, \sigma_{1}, p, a\right)-\phi_{i}\left(N, \sigma_{0}, p, a\right) \\
= & \phi_{j}\left(N, \sigma_{0}, p, a\right)-\phi_{j}\left(N, \sigma_{1}, p, a\right)-g_{i j}^{r} \\
= & \phi_{j}\left(N, \sigma_{1}, p, a\right)-\phi_{j}\left(N, \sigma_{0}, p, a\right) .
\end{aligned}
$$

It follows from (3.10) that

$$
\phi_{j}\left(N, \sigma_{1}, p, a\right)-\phi_{j}\left(N, \sigma_{0}, p, a\right)=\phi_{i}\left(N, \sigma_{1}, p, a\right)-\phi_{i}\left(N, \sigma_{0}, p, a\right)=-\frac{1}{2} g_{i j}^{r} .
$$

Hence, by induction assumption and (3.8), (3.9) we have

$$
\begin{aligned}
\phi_{i}\left(N, \sigma_{0}, p, a\right) & =\phi_{i}\left(N, \sigma_{1}, p, a\right)+\frac{1}{2} g_{i j}^{r} \\
& =\Gamma_{i}\left(N, \sigma_{1}, p, a\right)+\frac{1}{2} g_{i j}^{r} \\
& =\Gamma_{i}\left(N, \sigma_{0}, p, a\right) .
\end{aligned}
$$

We can show $\phi_{j}\left(N, \sigma_{0}, p, a\right)=\Gamma_{j}\left(N, \sigma_{0}, p, a\right)$ by the same way. Hence, we have $\phi\left(N, \sigma_{0}, p, a\right)=\Gamma\left(N, \sigma_{0}, p, a\right)$. 


\subsubsection{Relationships between the $\Gamma$-rule and the $\beta$-rule}

Let $(N, v)$ be a cooperative game and $\sigma_{0}$ an initial order of players. The $\beta$ rule [23] is defined as follows:

$$
\beta_{i}(v)=\frac{1}{2}\left[v\left(\bar{P}\left(\sigma_{0}, i\right)\right)-v\left(P\left(\sigma_{0}, i\right)\right)+v\left(\bar{F}\left(\sigma_{0}, i\right)\right)-v\left(F\left(\sigma_{0}, i\right)\right)\right],
$$

for all $i \in N$. Curiel et al. [23] showed that if $(N, v)$ is $\sigma_{0}$-component additive, then the $\beta$-rule is in the core of $(N, v)$.

Since the LE sequencing game $(N, v)$ is $\sigma_{0}$-component additive, we can deduce that $\beta(v)$ must be in the core of $(N, v)$. Since the $\Gamma$-rule gives a core element of $(N, v)$, too, we next discuss the relationships between these two allocation rules. The following theorem provides two conditions for the coincidence of the $\Gamma$-rule and the $\beta$-rule.

Theorem 3.11. Let $\left(N, \sigma_{0}, p, a\right)$ be an $L E$ sequencing situation and $(N, v)$ the corresponding LE sequencing game.

(i) When $a=0, \Gamma\left(N, \sigma_{0}, p, a\right)=\beta(v)$.

(ii) When $a<0, \Gamma\left(N, \sigma_{0}, p, a\right)=\beta(v)$ if and only if there are no three players $i, j, k \in N$ satisfying $\sigma_{0}(i)<\sigma_{0}(j)<\sigma_{0}(k)$ and $p_{i}>p_{j}>p_{k}$.

Proof. (i) Obviously, the LE sequencing games coincide with the sequencing games defined by Curiel et al. [21] and all LE-EGS rules coincide with the EGS rule when $a=0$. Since Curiel et al. [22] have shown that the EGS rule is equivalent to the $\beta$-rule in sequencing games, the result follows.

(ii) For any three orders $\sigma_{1}, \sigma_{2}, \sigma_{3} \in \Pi_{N}$, any $P e_{1}=\left\{\tau_{1}, \ldots, \tau_{m}\right\} \in \mathscr{P}\left(\sigma_{1}, \sigma_{2}\right)$ and $P e_{2}=\left\{\tau_{1}^{\prime}, \ldots, \tau_{k}^{\prime}\right\} \in \mathscr{P}\left(\sigma_{2}, \sigma_{3}\right)$, we define the connection of $P e_{1}$ and $P e_{2}$ by

$$
P e_{1} \triangleright P e_{2}=\left\{\tau_{1}, \ldots, \tau_{m}, \tau_{1}^{\prime}, \ldots, \tau_{k}^{\prime}\right\} .
$$

Clearly, $P e_{1} \triangleright P e_{2}\left(\sigma_{1}\right)=\sigma_{3}$.

(If). Fix any $i \in N$, and assume $\sigma_{0}(i)=r$. Recall the definitions of $U\left(\sigma_{0}, i\right)$ and $D\left(\sigma_{0}, i\right)$. For convenience, we denote $P\left(\sigma_{0}, i\right), \bar{P}\left(\sigma_{0}, i\right), F\left(\sigma_{0}, i\right)$, and $\bar{F}\left(\sigma_{0}, i\right)$ by $P_{i}, \bar{P}_{i}, F_{i}$, and $\bar{F}_{i}$. 
Case 1: Suppose that $U\left(\sigma_{0}, i\right)=\emptyset$. Let $\tau_{m}$ be the adjacent exchange for players $i$ and $d_{m}$ with $i$ being at the $(r+m-1)$-th position, where $1 \leq m \leq h_{2}$. Since there are no three players $i, j, k \in N$ satisfying $\sigma_{0}(i)<\sigma_{0}(j)<\sigma_{0}(k)$ and $p_{i}>p_{j}>p_{k}$, it follows that $p_{d_{1}} \leq \ldots \leq p_{d_{h_{2}}}$. Then we must have for any $P e \in \mathscr{P}\left(\sigma_{0}, \sigma_{F_{i}}\right), P e \triangleright\left\{\tau_{1}, \ldots, \tau_{h_{2}}\right\} \in \mathscr{P}\left(\sigma_{0}, \sigma_{\bar{F}_{i}}\right)$. From Lemma 3.7, it holds that

$$
v\left(\bar{F}_{i}\right)-v\left(F_{i}\right)=\sum_{m=1}^{h_{2}} g\left(\tau_{m}, \sigma_{m}, i, d_{m}\right)=\sum_{m=1}^{h_{2}} g_{i d_{m}}^{r+m-1},
$$

where $\sigma_{1}=\sigma_{F_{i}}$. Furthermore, since $U\left(\sigma_{0}, i\right)=\emptyset$, we have that

$$
v\left(\bar{P}_{i}\right)-v\left(P_{i}\right)=0 .
$$

It follows from (3.7),(3.11) and (3.12) that

$$
\begin{aligned}
\beta_{i}(v) & =\frac{1}{2}\left[v\left(\bar{P}_{i}\right)-v\left(P_{i}\right)+v\left(\bar{F}_{i}\right)-v\left(F_{i}\right)\right] \\
& =\frac{1}{2} \sum_{m=1}^{h_{2}} g_{i d_{m}}^{r+m-1} \\
& =\Gamma_{i}\left(N, \sigma_{0}, p, a\right) .
\end{aligned}
$$

Case 2: Suppose that $U\left(\sigma_{0}, i\right) \neq \emptyset$. Then there is at least one player in $P\left(\sigma_{0}, i\right)$ whose nominal processing time is larger than $p_{i}$. Since there are no three players $i, j, k \in N$ satisfying $\sigma_{0}(i)<\sigma_{0}(j)<\sigma_{0}(k)$ and $p_{i}>p_{j}>p_{k}$, we must have that $D\left(\sigma_{0}, i\right)=\emptyset$. Clearly, it follows that

$$
v\left(\bar{F}_{i}\right)-v\left(F_{i}\right)=0 .
$$

Let $\tau_{m}$ be the adjacent exchange for players $u_{m}$ and $i$ with $i$ being at the $(r-m+1)$-th position, where $1 \leq m \leq h_{1}$. Then for any $P e \in \mathscr{P}\left(\sigma_{0}, \sigma_{P_{i}}\right)$, we must have Pe $\triangleright\left\{\tau_{1}, \ldots, \tau_{h_{1}}\right\} \in \mathscr{P}\left(\sigma_{0}, \sigma_{\bar{P}_{i}}\right)$. From Lemma 3.7, it holds that

$$
v\left(\bar{P}_{i}\right)-v\left(P_{i}\right)=\sum_{m=1}^{h_{1}} g\left(\tau_{m}, \sigma_{m}, u_{m}, i\right)=\sum_{m=1}^{h_{1}} g_{u_{m} i}^{r-m},
$$


where $\sigma_{1}=\sigma_{P_{i}}$. Then it follows from (3.7),(3.13) and (3.14) that

$$
\begin{aligned}
\beta_{i}(v) & =\frac{1}{2}\left[v\left(\bar{P}_{i}\right)-v\left(P_{i}\right)+v\left(\bar{F}_{i}\right)-v\left(F_{i}\right)\right] \\
& =\frac{1}{2} \sum_{m=1}^{h_{1}} g_{u_{m} i}^{r-m} \\
& =\Gamma_{i}\left(N, \sigma_{0}, p, a\right) .
\end{aligned}
$$

(Only if). We prove this part by contradiction. Suppose $\Gamma\left(N, \sigma_{0}, p, a\right)=$ $\beta(v)$ and there are three players $i, j, k \in N$ satisfying $\sigma_{0}(i)<\sigma_{0}(j)<\sigma_{0}(k)$ and $p_{i}>p_{j}>p_{k}$. Define

$$
\begin{gathered}
i^{*}=\arg \min \left\{\sigma_{0}(i) \mid \exists j, k \in N \text { such that } \sigma_{0}(i)<\sigma_{0}(j)<\sigma_{0}(k)\right. \\
\text { and } \left.p_{i}>p_{j}>p_{k}\right\} .
\end{gathered}
$$

It is easy to see that then, $U\left(\sigma_{0}, i^{*}\right)=\emptyset$. Let $D\left(\sigma_{0}, i^{*}\right)=\left\{d_{1}, \ldots, d_{h_{2}}\right\}=$ $\left\{d_{1}^{\prime}, \ldots, d_{h_{2}}^{\prime}\right\}$ be two orderings of $D\left(\sigma_{0}, i^{*}\right)$ with $\sigma_{0}\left(d_{1}\right)<\cdots<\sigma_{0}\left(d_{h_{2}}\right)$ and with $p_{d_{1}^{\prime}} \leq \cdots \leq p_{d_{h_{2}}^{\prime}}$. Assume that $i^{*}$ is in position $r^{*}$ in $\sigma_{0}$. We first show that

$$
\sum_{m=1}^{h_{2}} g_{i^{*} d_{m}}^{r^{*}+m-1}<\sum_{m=1}^{h_{2}} g_{i^{*} d_{m}^{\prime}}^{r^{*}+m-1} .
$$

What (3.15) expresses is that the benefits of successive adjacent exchanges of $i^{*}$ with jobs in $D\left(\sigma_{0}, i^{*}\right)$ is larger, if the jobs in $D\left(\sigma_{0}, i^{*}\right)$ are ordered by nominal processing times (SPT).

To prove (3.15), it suffices to show that the two successive adjacent exchanges $\left(i^{*}, k\right)$ and $\left(i^{*}, j\right)$ (in this order) are better than $\left(i^{*}, j\right)$ and $\left(i^{*}, k\right)$, whenever $p_{j}>p_{k}$. Formally, this is expressed by

$$
g_{i^{* j}}^{r}+g_{i^{*} k}^{r+1}<g_{i^{*} k}^{r}+g_{i^{* j}}^{r+1} \text { for any } r^{*} \leq r \leq n-1 .
$$


But this is true, as

$$
\begin{aligned}
g_{i^{*} k}^{r}-g_{i^{* j}}^{r}+g_{i^{*} j}^{r+1}-g_{i^{*} k}^{r+1}= & \theta(r)\left(p_{i^{*}}-p_{k}\right)-\theta(r)\left(p_{i^{*}}-p_{j}\right) \\
& +\theta(r+1)\left(p_{i^{*}}-p_{j}\right)-\theta(r+1)\left(p_{i^{*}}-p_{k}\right) \\
= & \theta(r)\left(p_{j}-p_{k}\right)+\theta(r+1)\left(p_{k}-p_{j}\right) \\
= & {[\theta(r)-\theta(r+1)]\left(p_{j}-p_{k}\right) } \\
> & 0,
\end{aligned}
$$

where $\theta(r)$ is defined in (3.2). Note that the inequality in (3.15) is strict, because of the choice of $i^{*}$, and since this implies the existence of $j, k$ with $\sigma_{0}\left(i^{*}\right)<\sigma_{0}(j)<\sigma_{0}(k)$ and $p_{i^{*}}>p_{j}>p_{k}$.

Therefore, (3.16) holds, and it follows that (3.15) holds. Let $\tau_{m}$ be the adjacent exchange for players $i^{*}$ and $d_{m}^{\prime}$ with $i^{*}$ being at the $(r+m-1)$-th position, where $1 \leq m \leq h_{2}$. Then for any $P e \in \mathscr{P}\left(\sigma_{0}, \sigma_{F_{i^{*}}}\right), P e \triangleright\left\{\tau_{1}, \ldots, \tau_{h_{2}}\right\} \in$ $\mathscr{P}\left(\sigma_{0}, \sigma_{\bar{F}_{i^{*}}}\right)$. Similar to (3.11), we have

$$
v\left(\bar{F}_{i^{*}}\right)-v\left(F_{i^{*}}\right)=\sum_{m=1}^{h_{2}} g\left(\tau_{m}, \sigma_{m}, i^{*}, d_{m}^{\prime}\right)=\sum_{m=1}^{h_{2}} g_{i^{*} d_{m}^{\prime}}^{r^{*}+m-1},
$$

where $\sigma_{1}=\sigma_{F_{i^{*}}}$. Since $U\left(\sigma_{0}, i^{*}\right)=\emptyset$, we have that

$$
v\left(\bar{P}_{i^{*}}\right)-v\left(P_{i^{*}}\right)=0 .
$$

Thus,

$$
\begin{aligned}
\beta_{i^{*}}(v) & =\frac{1}{2}\left[v\left(\bar{P}_{i^{*}}\right)-v\left(P_{i^{*}}\right)+v\left(\bar{F}_{i^{*}}\right)-v\left(F_{i^{*}}\right)\right] \\
& =\frac{1}{2} \sum_{m=1}^{h_{2}} g_{i^{*} d_{m}^{\prime}}^{r^{*}+m-1} .
\end{aligned}
$$

From (3.7), we have that

$$
\Gamma_{i^{*}}\left(N, \sigma_{0}, p, a\right)=\frac{1}{2} \sum_{m=1}^{h_{2}} g_{i^{*} d_{m}}^{r^{*}+m-1} .
$$

Then it follows from (3.15) that $\beta_{i^{*}}(v)>\Gamma_{i^{*}}\left(N, \sigma_{0}, p, a\right)$, a contradiction. 
We can immediately conclude the following corollary from (3.14) in the proof of Theorem 3.11.

Corollary 3.1. For LE sequencing situation $\left(N, \sigma_{0}, p, a\right)$, let $(N, v)$ be the corresponding LE sequencing game and $i_{n}=\sigma_{0}^{-1}(n)$ be the last player in $\sigma_{0}$. Then $\Gamma_{i_{n}}\left(N, \sigma_{0}, p, a\right)=\beta_{i_{n}}(v)$.

\subsection{Extensions}

One of the limitations of this chapter is the restriction of the sequencing model to learning indices and cost functions which are identical for all players. We briefly discuss extensions of the model to these more general cases.

First, assume different players have different learning indices (but identical cost functions). That is, any player $i \in N$ will have her own learning index $a_{i}$. We can define an $L E^{a}$ sequencing situation by a 4-tuple $\left(N, \sigma_{0}, p, \bar{a}\right)$, where $\bar{a}=\left(a_{i}\right)_{i \in N} \in \mathbb{R}_{\leq 0}^{n}$. Then the corresponding $L E^{a}$ sequencing game $\left(N, u^{\lambda}\right)$ can be defined by letting, for all $S \subseteq N$,

$$
v_{a}^{\lambda}(S)=\max _{\sigma \in \Pi_{S}}\left\{c_{\sigma_{0}}^{\prime}(S)-c_{\sigma}^{\prime}(S)+\lambda(S)\left[c_{\sigma_{0}}^{\prime}(N \backslash S)-c_{\sigma}^{\prime}(N \backslash S)\right]\right\} .
$$

where $c_{\sigma}^{\prime}(S)=\sum_{i \in S} \sum_{\sigma_{0}(j) \leq \sigma_{0}(i)} \sigma_{0}(i)^{a_{i}} p_{i}$. Mosheiov et al. [60] showed that the problem of finding the optimal order for the $\mathrm{LE}^{a}$ sequencing situation can be solved in $O\left(n^{3}\right)$ by formulating it as an assignment problem. By applying the same method, we can also prove that the optimal order for obtaining $v_{a}^{\lambda}(S)$ can be found in polynomial time. Thus, $v_{a}^{\lambda}(S)$ for any $S$ is well-defined. Let $\left(N, v_{a}\right)$ be the $\mathrm{LE}^{a}$ sequencing game with the share function $\lambda$ satisfying for $S \subseteq N, \lambda(S)=1$. One can easily verify that $\left(N, v_{a}\right)$ is $\sigma_{0}$-component additive, and thus any corresponding $\mathrm{LE}^{a}$ sequencing game is balanced and has a non-empty core. However, it is not clear how to extend the EGS rule to $\mathrm{LE}^{a}$ sequencing situations, since there is no fixed principle for the optimal order.

Second, consider the possibility to allow different players to have different cost coefficients but identical learning indices. An $L E^{w}$ sequencing situation is then described by a 5 -tuple $\left(N, \sigma_{0}, w, p, a\right)$, where $w=\left(w_{i}\right)_{i \in N} \in \mathbb{R}_{\geq 0}^{n}$, which 
is called cost coefficients or weights. That is to say, the cost of player $i \in N$ is now $w_{i} C(\sigma, i)$. Analogous to the definition of LE sequencing games, we can define the corresponding $L E^{w}$ sequencing game $\left(N, y^{\lambda}\right)$ by letting, for all $S \subseteq N$,

$$
v_{w}^{\lambda}(S)=\max _{\sigma \in \Pi_{S}}\left\{c_{\sigma_{0}}^{*}(S)-c_{\sigma}^{*}(S)+\lambda(S)\left[c_{\sigma_{0}}^{*}(N \backslash S)-c_{\sigma}^{*}(N \backslash S)\right]\right\},
$$

where $c_{\sigma}^{*}(S)=\sum_{i \in S} w_{i} \sum_{\sigma_{0}(j) \leq \sigma_{0}(i)} \sigma_{0}(i)^{a} p_{i}$.

However, finding an optimal order for minimizing the total costs of all players in an $\mathrm{LE}^{w}$ sequencing situation may be NP-hard in general, in which case there is not much hope to find a closed-form for working with $v_{w}^{\lambda}(S)$. One way to circumvent this problem is to restrict to $\mathrm{LE}^{w}$ sequencing situations to the case with agreeable weights, which says that for any $i, j \in N, p_{i} \leq p_{j}$ implies $w_{i} \geq w_{j}$. Zhao et al. [94] showed that the total costs of all players in an $\mathrm{LE}^{w}$ sequencing situation with agreeable weights can be minimized by the WSPT rule. In this case, one can work with closed-form expressions for $v_{w}^{\lambda}(S)$, like in the first part of this chapter. However, the agreeability condition is quite strong.

\subsection{The PoA of noncooperative games for $\mathrm{LE}^{w}$ sequenc- ing situations}

As we mentioned in the previous section, finding the optimal order for minimizing the total costs of all players in an LE sequencing situation where players have different cost coefficients is difficult, and as a matter of fact, it is still an open problem (see Section 3.6 for a brief discussion). Therefore, building a framework as a cooperative game for $\mathrm{LE}^{w}$ sequencing situations seems impossible for now.

Instead, we turn to the following structural question: inspired by the pairwise exchange process of players that was key in the earlier sections, is there a "stable" assignment for all players that is not too far from the optimal one? What arrangements will the players make about their positions in the queue 
if they are allowed to switch with their direct predecessor or successor, sharing the potential benefits of such a switch? With these questions in mind, we design a noncooperative game for the $\mathrm{LE}^{w}$ sequencing situation that lets the players decide their own strategies of exchanging positions and any agreement can only be operated if it is self-enforcing.

The game processes in rounds. In each round, any pair of neighboring players may switch their positions if they can realize cost savings by doing so. The cost saving for the two involved players will be equally divided between them.

The game ends if there is no switch in one round that would benefit the players, i.e., the sequence is "locally stable". The final order can be seen as an equilibrium state since no player can get benefit by switching positions with his or her neighbors. In this section, we define the above game as $S G(N)$, and call the final order of players in the noncooperative game a stable order.

Note that cost savings for the two switching players we define in this section do not include the cost changes of succeeding players, which is different from the previous sections. This can be interpreted as the myopic actions of players when they are making noncooperative decisions. It can be motivated by the assumption that the players only have "local" information. On the other hand, this feature in the noncooperative game reflects a situation where people value their personal benefits over the social effects.

For any two neighboring jobs $i, j$, assume that $i$ is in position $r, j$ in $r+1$ and the start time of $i$ is $t$. They will not exchange positions if

$$
\begin{aligned}
& \left(t+p_{i} r^{a}\right) w_{i}+\left[t+p_{i} r^{a}+p_{j}(r+1)^{a}\right] w_{j} \\
& \leq\left(t+p_{j} r^{a}\right) w_{j}+\left[t+p_{j} r^{a}+p_{i}(r+1)^{a}\right] w_{i} .
\end{aligned}
$$

The left side of inequality (3.17) is the weighted costs of $i, j$ before they exchange positions, and the right side is the weighted costs of $i, j$ after the switch. That is, we assume a switch only happens if the benefit is larger than 0 . If we simplify (3.17) we get

$$
\left(p_{i}-p_{j}\right)\left(w_{i}+w_{j}\right) r^{a}+\left(p_{j} w_{j}-p_{i} w_{i}\right)(r+1)^{a} \leq 0 .
$$




\subsubsection{The existence of stable orders}

The first question we should care about is if stable orders always exist for any sequencing instance with learning effect. Indeed, there could exist instances in which the switching process will never end. In the following, we will show that stable orders always exist. But first, we need the following notations and lemmas.

Given any $S G(N)$, we define the corresponding directed graph $G(\Sigma, E)$, where $\Sigma$ is the set of vertices and $E$ is the set of directed edges. Each vertex represents a possible order of players in $S G(N)$. Denote $e=\left(\sigma, \sigma^{\prime}\right)$ as a directed edge from $\sigma$ to $\sigma^{\prime}$ if $\sigma^{\prime}$ can be obtained by a beneficial adjacent switch from $\sigma$. A directed trail is a sequence of distinct directed edges $\left(e_{1}, \cdots, e_{n-1}\right)$ for which there is a sequence of vertices $\left(\sigma_{1}, \cdots, \sigma_{n}\right)$, such that for $i=$ $1, \cdots, n-1, e_{i}=\left(\sigma_{i}, \sigma_{i+1}\right)$. A directed cycle is a directed trail in which only the first vertex is repeated, i.e., $\sigma_{1}=\sigma_{n}$. A directed graph without directed cycle is called a directed acyclic graph. A 2-cycle is a directed cycle with only two directed edges. A graph having no 2-cycle is called an oriented graph.

It is easy to observe that a $S G(N)$ must have stable orders if the corresponding directed graph is a directed acyclic graph. Given any $S G(N)$, the corresponding directed graph $G(\Sigma, E)$ is an oriented graph. This is because, after a beneficial adjacent switch, the two players involved can not directly switch back, otherwise, they will have non-positive cost savings. Therefore, any directed cycle must have at least 3 directed edges, in which there must be at least 3 players switching with each other at least twice.

Lemma 3.12. Given a $S G(N)$, suppose that $i, j$ would switch in positions $r, r+1$ with $i$ in position $r$, and they also would switch in positions $m, m+1$ with $j$ in position $m$. If $r<m$, then we have $p_{i}>p_{j}$.

Proof. Since any switch between two neighboring players will only happen if their cost savings are positive, we must have the following inequalities:

$$
\begin{aligned}
& p_{i} r^{a} w_{i}+\left(p_{i} r^{a}+p_{j}(r+1)^{a}\right) w_{j} \\
& -\left[p_{j} r^{a} w_{j}+\left(p_{j} r^{a}+p_{i}(r+1)^{a}\right) w_{i}\right]>0,
\end{aligned}
$$


and

$$
\begin{aligned}
& p_{j} m^{a} w_{j}+\left(p_{j} m^{a}+p_{i}(m+1)^{a}\right) w_{i} \\
& -\left[p_{i} m^{a} w_{i}+\left(p_{i} m^{a}+p_{j}(m+1)^{a}\right) w_{j}\right]>0 .
\end{aligned}
$$

Combining (3.19) and (3.20) we get

$$
\left(\frac{r+1}{r}\right)^{a}\left(p_{i} w_{i}-p_{j} w_{j}\right)<\left(p_{i}-p_{j}\right)\left(w_{i}+w_{j}\right)<\left(\frac{m+1}{m}\right)^{a}\left(p_{i} w_{i}-p_{j} w_{j}\right) .
$$

Since $m>r$, we have $\left(\frac{m+1}{m}\right)^{a}>\left(\frac{r+1}{r}\right)^{a}$. So $p_{i} w_{i}-p_{j} w_{j}>0$. Therefore, we have $p_{i}>p_{j}$.

Theorem 3.13. For any sequencing game $S G(N)$, there exist at least one stable order.

Proof. Suppose that there is a $S G(N)$ without a stable order. Then there must exist at least one directed cycle in the corresponding directed graph. Pick any directed cycle $\mathscr{C}, \sigma_{1}$ as any vertex of $\mathscr{C}$ and $S$ be the set of all players who switch in $\mathscr{C}$.

Let $k$ be the last player of $S$ in $\sigma_{1}$. In the directed cycle $\mathscr{C}$, there will be a player $j \in S$ who will first switch with $k$. Since $k$ is the last player of $S$ in $\sigma_{1}$, he can only switch back in earlier positions. According to Lemma 3.12, we therefore have $p_{k}>p_{j}$.

Now, $j$ is in the last position of $S$. There will be another player $i \in S \backslash\{k\}$ who will first switch with $j$. Since $j$ is the last player of $S$ in the current order, players $i, j$ can only switch back in earlier positions. From Lemma 3.12, we have $p_{j}>p_{i}$. Continuing the above process and assuming that $m$ is the last player of $S$ when $k$ reaches the position just before $m$. Since $k$ has to return to his original position $\sigma_{1}(k)$ in $\mathscr{C}, k$ and $m$ now have to switch. Then we get a series of players satisfying

$$
p_{k}>p_{j}>p_{i}>\cdots>p_{m}>p_{k}
$$

a contradiction. 


\subsubsection{Lower and upper bound on the PoA}

In this subsection, we investigate how far away any stable order can be from an optimal order. We borrow the concept of the Price of Anarchy (PoA) to measure how efficient $S G(N)$ is when it is compared with the cooperative behavior, which would simply compute the optimal order. However, as we mentioned several times before, finding an algorithm for calculating an optimal order for $\mathrm{LE}^{w}$ sequencing situations is still an open problem. Therefore, the best way to show the efficiency of $S G(N)$ is to analyze the range of the PoA.

Given a $S G(N)$, we denote $\sigma_{s t}$ as a stable order of $S G(N), \Pi_{s t}$ the set of all stable orders of $S G(N), \sigma_{o p t}$ as an optimal order. Define the worst stable order as $\sigma_{w s t}=\arg \max _{\sigma_{s t} \in \Pi_{s t}} c_{\sigma_{s t}}(N)$.

The PoA of a $S G(N)$ is the ratio between the total weighted costs in the worst stable order and in the optimal order, as formally defined below.

Definition 3.1. Given any $S G(N)$, the PoA of $S G(N)$ is defined as follows:

$$
\operatorname{PoA}(S G(N))=\frac{c_{\sigma_{w s t}}(N)}{c_{\sigma_{o p t}}(N)} .
$$

Let $\mathscr{L}$ be a set of $S G(N)$. Given any $\mathscr{L}$, the PoA of $\mathscr{L}$ is defined as

$$
\operatorname{PoA}(\mathscr{L})=\sup _{S G(N) \in \mathscr{L}} \operatorname{PoA}(S G(N))
$$

For any instance $S G(N)$, we denote the ratio between the total weighted costs in reversed WSPT order and in WSPT order, both without considering the learning effect as $B(S G(N))$. Formally,

$$
B(S G(N))=\frac{p_{n} \sum_{i=1}^{n} w_{i}+p_{n-1} \sum_{i=1}^{n-1} w_{i}+\cdots+p_{1} w_{1}}{p_{1} \sum_{i=1}^{n} w_{i}+p_{2} \sum_{i=2}^{n} w_{i}+\cdots+p_{n} w_{n}} .
$$

Given any $\mathscr{L}$, let $B(\mathscr{L})=\max _{S G(N) \in \mathscr{L}} B(S G(N))$.

For notational simplicity, in the rest of this section, the total weighted costs of all players in order $\sigma$ will be written as $c(\sigma)$. The following theorem gives an upper bound on the PoA. 
Theorem 3.14. Give any set $\mathscr{L}$ of $S G(N)$ for $L E^{w}$ sequencing situations, we have

$$
\operatorname{Po} A(\mathscr{L}) \leq B(\mathscr{L}) n^{-a} .
$$

Proof. Given any $S G(N)$, let $\sigma_{\text {wspt }}$ be the WSPT order. Denote $c_{0}(\sigma)$ as the total weighted costs without learning effect in order $\sigma$. It is easy to see that for any stable order $\sigma_{s t}$

$$
c\left(\sigma_{s t}\right) \leq c_{0}\left(\sigma_{s t}\right) \leq c_{0}\left(\sigma_{w}\right)
$$

where $\sigma_{w}=\arg \max _{\sigma \in \Pi} c_{0}(\sigma)$. For sequencing situations without learning effect, the worst schedule is the reversed WSPT rule. Therefore,

$$
\frac{c_{0}\left(\sigma_{w}\right)}{c_{0}\left(\sigma_{w s p t}\right)}=B(S G(N))
$$

In a sequencing situation with learning effect, the only job whose nominal processing time will be decreased by $n^{a}$ is the last job. So, we get

$$
n^{a} c_{0}\left(\sigma_{w s p t}\right) \leq n^{a} c_{0}\left(\sigma_{o p t}\right) \leq c\left(\sigma_{o p t}\right)
$$

From (3.21), (3.22) and (3.23) we get for any stable order $\sigma_{s t}$

$$
\frac{c\left(\sigma_{s t}\right)}{c\left(\sigma_{o p t}\right)} \leq B(S G(N)) n^{-a} \text {. }
$$

Thus, for any $S G(N), \operatorname{Po} A(S G(N)) \leq B(S G(N)) n^{-a}$, which implies the result.

Note that $B(\mathscr{L})$ could be arbitrarily large, hence the upper bound on the PoA could also be arbitrarily large.

In the following we will show by the following example a lower bound on the PoA.

Example 3.3. Considering the game $S G(N)$, with the nominal processing times $\left(1,\left(\frac{n}{n-1}\right)^{a} \epsilon^{-1},\left(\frac{n}{n-2}\right)^{a} \epsilon^{-2}, \cdots\right)$. That is, for any $k \in N, p_{k}=\left(\frac{n}{n-k+1}\right)^{a} \epsilon^{1-k}$, where $\epsilon>0$ is an arbitrary small number. The weights of players are $w=$ 
$\left(M^{n}, M^{n-1}, \cdots, 1\right)$, and $M$ is an arbitrary large number. Now, we have the following claims.

Claim 1 The order $(1, \cdots, n)$ is stable, if $\epsilon$ is small enough.

Proof. Consider two arbitrary neighboring jobs $k, k+1$. To insure stability, from (3.18), we must guarantee the following inequality hold:

$$
\left(p_{k}+p_{k+1}\right)\left(M^{n+1-k}+M^{n-k}\right) k^{a}+\left(p_{k+1} M^{n-k}-p_{k} M^{n+1-k}\right)(k+1)^{a} \leq 0 .
$$

Define $z_{k}=\left(\frac{k}{k+1}\right)^{a}$. Note that $z_{k}>1$ for any $k$. Then the above inequality is equal to:

$$
\begin{aligned}
\frac{p_{k}}{p_{k+1}} & \leq \frac{\left(M^{n+1-k}+M^{n-k}\right) k^{a}-M^{n+1-k}(k+1)^{a}}{\left(M^{n+1-k}+M^{n-k}\right) k^{a}-M^{n+1-k}(k+1)^{a}} \\
& =\frac{(M+1) k^{a}-(k+1)^{a}}{(M+1) k^{a}-M(k+1)^{a}} \\
& =\frac{(M+1) z_{k}-1}{(M+1) z_{k}-M} \\
& =\frac{M z_{k}+z_{k}-1}{M\left(z_{k}-1\right)+z_{k}} .
\end{aligned}
$$

For any $k \in N, \frac{p_{k}}{p_{k+1}}=\left(\frac{n-k}{n-k+1}\right)^{a} \epsilon$. Since $\epsilon$ can be arbitrarily small, we must have $\frac{p_{k}}{p_{k+1}} \leq \frac{M z_{k}+z_{k}-1}{M\left(z_{k}-1\right)+z_{k}}$ if $\epsilon \leq \frac{M z_{k}+z_{k}-1}{M\left(z_{k}-1\right)+z_{k}}\left(\frac{n-k+1}{n-k}\right)^{a}$.

Claim 2 Given any $\delta>0$, the total weighted costs in an optimal order are no larger than $(1+\delta) n^{a} M^{n}$, if $\epsilon$ is small enough and $M$ is large enough.

Proof. If the order is $\sigma=(n, \cdots, 1)$, the completion time of job 1 in this order will be

$$
\begin{aligned}
& \epsilon^{-(n-1)} n^{a}+\epsilon^{-(n-2)} n^{a}+\cdots+n^{a} \\
= & \left.\left(1+\epsilon^{(}-1\right)+\epsilon^{-2}+\cdots+\epsilon^{-(n-1)}\right) n^{a} \\
= & \left(\frac{1-\epsilon^{n}}{1-\epsilon}\right) n^{a} .
\end{aligned}
$$


Thus,

$$
\begin{aligned}
c(\sigma) & \leq(n-1)\left(\frac{1-\epsilon^{n}}{1-\epsilon}\right) n^{a} M^{n-1}+\left(\frac{1-\epsilon^{n}}{1-\epsilon}\right) n^{a} M^{n} \\
& =\left(\frac{M+n-1}{M}\right)\left(\frac{1-\epsilon^{n}}{1-\epsilon}\right) n^{a} M^{n} .
\end{aligned}
$$

If we choose $M$ to be large enough and $\epsilon$ to be small enough, then for any $\delta>0$, the following inequality must hold,

$$
\frac{M+n-1}{M} \leq(1+\delta)\left(\frac{1-\epsilon}{1-\epsilon^{n}}\right)
$$

Combining (3.24) and (3.25), we have $c\left(\sigma_{\text {opt }}\right) \leq c(\sigma) \leq(1+\delta) n^{a} M^{n}$.

Claim 3 Given any $\delta>0$,

$$
\operatorname{PoA}(S G(N)) \geq\left(\frac{1}{1+\delta}\right) n^{-a}
$$

Proof. Let $\sigma_{s t}=(1, \cdots, n)$ be the stable order from Claim 1. From Claim 2 we have

$$
P o A(S G(N)) \geq \frac{c\left(\sigma_{s t}\right)}{c(\sigma)} \geq \frac{M^{n}}{(1+\delta) n^{a} M^{n}}=\left(\frac{1}{1+\delta}\right) n^{-a} .
$$

The following theorem follows immediately from Claim 3 in the above example when $\delta$ goes to 0 .

Theorem 3.15. Let $\mathscr{L}$ be the set of $S G(N)$. The we have

$$
\operatorname{PoA}(\mathscr{L}) \geq n^{-a} \text {. }
$$

Remark 3.1. Theorem 3.14 and Theorem 3.15 indicate that the efficiency of the noncooperative games for $\mathrm{LE}^{w}$ sequencing situations mostly relies on the coefficients of the number of players and the learning effect. The smaller the number of players or closer the learning effect to 0 , the more efficient the stable solutions are, relative to the optimal solutions However, if $N$ is very 
large or $a$ is much smaller than 0 , the myopic strategies of players could lead the stable solutions far away from the optimal solutions.

\subsection{Computational complexity of sequencing with learn- ing}

This section is devoted to a brief discussion of the computational complexity of sequencing problems with position-dependent learning in general. To that end, we define the following generalization of the problem defined in Section 3.4.

Definition 3.2. (Scheduling problem with general learning function) A scheduling problem with a genral learning function is described by a 4-tuple $(N, w, p, f)$, where $f:\{1, \cdots, n\} \rightarrow[0,1]$ is a position-dependent monotonically decreasing function. The actual processing time of job $j$ when it is in position $r$ is $p_{j}(r)=p_{j} f(r)$. Let $C(\sigma, j)$ be the completion time of job $j$ in order $\sigma$. The objective is to find an optimal order so that the following function is minimized

$$
\sum_{j \in N} w_{j} C(\sigma, j) .
$$

As a special case, let us consider the following problem.

Definition 3.3. (Scheduling problem with a 0-1 learning function) A scheduling problem with a 0-1 learning function is a scheduling problem with a general learning function, where $f(r)=1$ for $k=1, \ldots, k$ and $f(r)=0$ for $r=$ $k+1, \ldots, n$.

This problem boils down to select a subset $S$ of $k$ jobs to be sequenced on a single machine, while the remaining $n-k$ jobs are all scheduled at time $\sum_{i \in S} p_{i}$, as their processing times are equal to 0 . As it is optimal to schedule the jobs $S$ in the WSPT order, let us denote by $W S P T(S)$ the minimal total weighted completion time of $S$ on a single machine. Then, the special case just defined boils down to select a set $S$ of $k$ jobs to minimize the objective function

$$
W S P T(S)+p(S) w(N \backslash S) .
$$


Here, as usual we denote by $w(N \backslash S)=\sum_{j \in N \backslash S} w_{j}$ the total weights of jobs $N \backslash S$, and likewise, $p(S)=\sum_{j \in S} p_{j}$.

If we can prove that this problem is NP-hard, then the scheduling problem with general learning function is also NP-hard. Notice that the problem with 0-1 learning function is very closely related to the following scheduling problem with rejection, which was also discussed in [26].

Definition 3.4. (Scheduling problem with rejection) A scheduling problem with rejection is described as follows: given a set of jobs $N$, and for each job, we have to decide either to schedule that job on a machine or reject it. Each job $j$ has a rejection cost $e_{j}$, a processing time $p_{j}$ and a weight $w_{j}$. If job $j$ is decided to schedule on the machine, the weighted completion time of $j$ is $w_{j} C_{j}$, where $C_{j}$ is denoted as the completion time of $j$. If job $j$ is rejected, the rejection cost $e_{j}$ is paid. The objective is to select a job set $S$ so as to minimize

$$
W S P T(S)+e(N \backslash S),
$$

where $e(N \backslash S)=\sum_{j \in N \backslash S} e_{j}$.

Engels et al. [26] showed that scheduling with rejection is (weakly) NPhard.

In a scheduling problem with a 0-1 learning function, if we predetermine the value $p(S)$, and set $e_{j}=p(S) w_{j}$, then we get the following problem, which is very similar to a scheduling problem with rejection, except that $|S|$ is fixed.

Definition 3.5. (Scheduling problem with k-rejection) A scheduling problem with $k$-rejection is a variation of the scheduling problem with rejection, in which the number of jobs to be rejected is fixed to be $k$, and the rejection cost of each job $j$ is fixed to be $e_{j}=e w_{j}$, for some constant $e$.

In fact, if we can solve scheduling problems with k-rejection in polynomial time, then we can solve scheduling problems with 0-1 learning function in pseudo-polynomial time by enumerating all the possible values $p(S)$ in integer interval $[0, p(N)]$. This means that if we can prove scheduling problems with $0-1$ learning function to be strongly NP-hard, we can prove that scheduling with k-rejection is also NP-hard. 
Given the close similarities of these two kinds of single machine scheduling problems, we have the following conjectures.

Conjecture 3.1. Scheduling problems with 0-1 learning function and scheduling problems with k-rejection are both weakly NP-hard.

Conjecture 3.2. Scheduling problems with a general learning functions are NP-hard.

Unfortunately, we have not been able to nail down the proofs of these two conjectures. 


\section{Chapter 4}

\section{Games in sequencing situations with externalities}

In this chapter, we consider cooperative games in sequencing situations with externalities, called partition sequencing games. In these games, the worth of a coalition can be influenced by external players in the queue. We first show that partition sequencing games satisfy cohesiveness and have non-positive externalities. Then by proposing partition rules, which are specifications on how external players should partition themselves, we study the relationships between the allocation given by the EGS rule and the cores based on different partition rules. We show that the EGS rule always yields a core element independent of the partition rule is applied to the game. Moreover, we propose a mechanism that implements the EGS rule for partition sequencing games.

\subsection{Introduction}

Cooperation in scheduling theory models represents an interesting research field on the frontier between game theory and scheduling problems. The research in this area deals with issues in which a finite number of agents, lined up in front of one or more machines, need to be processed, and these agents are faced with the decision-making problem of reducing their waiting 
costs by rearranging their orders. In the analysis of a sequencing model in this category, two questions that have aroused wide attention are: how to obtain the optimal order for the agents so as to minimize their total costs; and how the maximal cost savings should be allocated among the agents. Curiel et al. [21] first take a game theoretic approach for solving these problems. They introduce sequencing games arising from a single machine sequencing situation in which jobs are waiting in line to be processed in front of a single machine. In their setting, the worth of a coalition is seen as the maximal cost savings the coalition can achieve by admissible rearrangements of its members without jumping over non-members. They also introduced the EGS rule which generates a core element of the corresponding sequencing game. Since the pioneering work of Curiel et al. [21], different types of modifications and generalizations about sequencing games have been presented, including ready times [38], due dates [9], multiple machines [12, 39, 80], grouped jobs $[17,35]$, multistage situations $[18,19]$, relaxed sequencing games [62, 81, 84] and sequencing situations without an initial order [37, 51]. A review on sequencing games can be found in [20].

Up until now, a common feature among the researches of sequencing games and their extended models is that the cooperative game theoretic approach, taken to the scheduling problem, is rooted in characteristic function form (CFF), which inherently assumes that the worth of a coalition is independent of the structure of other players. The underlying assumption behind all the definitions of these sequencing games is: what a group of players in the queue can achieve by cooperating only relies on the maximal cost savings through admissible rearrangements of its own members. This implies that the influence of other coalitions on this achievement is neglected.

In this chapter, we take the impacts of external players' reactions into consideration for determining the worth of a coalition in sequencing situations and related cooperative games. For example, consider the case where a collaborative manufacturing chain is formed by several companies. If some of the members separate from the co-production process, the full cooperation agreement will certainly break and all the remaining companies are free to contract with each other. So now, in order to access its real value, the deviating coalition should focus on not only the cost savings obtained from the 
rearrangement of its members but also the mutual influences between it and other later-formed coalitions. In this chapter, these mutual influences which are regarded as mutual negotiations for trading places of coalitions are the key points of defining the worths of coalitions. That is, the existence of external factors implies that the evaluation of the worth of a coalition will be conducted on two stages: firstly, internal collaboration stage, and secondly, external negotiation stage. Hence, the final surplus for a coalition is mostly conditioned on the structure of external players. This idea happens to coincide with the well-known situation of externalities in coalitional game theory, which is first modeled by Thrall and Lucas [83] as partition function form (PFF) games. In such games, a coalition can have multiple worths depending on how the outside players partition themselves. In this chapter, we first introduce a class of sequencing games in partition function form by defining the worth of a coalition as the sum of surpluses generated from the two-stage process mentioned previously, and we call these games partition sequencing games. We will show that these games are cohesive (the grand coalition generates the largest total surplus) and have non-positive externalities (the merger between two disjoint coalitions does not make other coalitions better off).

For cooperative games, the core is a well-known solution concept and is widely regarded as an effective way to analyze the stability and fairness of coalitional games. There are already many attempts to define a modification of the core for PFF games, e.g., in [1], [8], [29], [36], [52]. The definitions of the core of partition function form games proposed in the literature are fundamentally based on pre-specified behavioral assumptions about the reactions of external players. Similarly, we introduce a concept called the partition rule, which is a mapping from a deviating coalition to a partition of the grand coalition containing the deviating coalition itself; namely, a partition rule specifies the reactions of external players to a deviation. Two specific partition rules are given: the m-exogenous rule and the s-exogenous rule. We will show that the best-case scenario for a deviating coalition is that external players behave according to the s-exogenous rule, and the worst-case scenario is that external players behave according to the m-exogenous rule. We then define the cores of partition function form games based on disparate partition rules and show that the EGS rule always yields a core allocation. 
Specially, the allocation generated by the EGS rule is the only core element if the s-exogenous rule is applied to a partition sequencing game.

While the EGS rule is a reasonable way of distributing the gains of the cooperation among players in sequencing situations, its noncooperative foundation has not been formally provided in the literature as far as we know. In the last part of this chapter, we focus on a noncooperative approach to shape the strategic behavior of individuals for the implementation of the EGS rule. The mechanism we propose in this chapter is a noncooperative sequential process in the same spirit of Rubinstein's [75] alternating offers bargaining game and its extensions to $n$ players by Chatterjee et al. [15]. The player in the first position in the queue initiates the game by proposing a coalition containing himself and offers to other players in that coalition. If all the respondents accept the proposal, the coalition is formed. Then the game is played among the remaining players. If at least one respondent rejects the proposal, the proposer is removed from the game and forms a singleton by himself. Then the first rejecter becomes the new proposer in the next period. This dynamic bargaining model is similar to the games proposed by Chatterjee et al. [15], Bloch [7], and Ray and Vohra. [73]. But the main differences between these games and ours are that first, we do not incorporate the factor of discounting; second, the proposer proposing an unaccepted proposal will certainly be removed from the game in the next round. We will show that the subgame perfect equilibrium outcomes of this mechanism coincide with the payoff vector yielded by the EGS rule.

The plan of the chapter is as follows. In Section 4.2 we introduce the partition sequencing games. It is shown that these games are cohesive and have non-positive externalities. In Section 4.3 the concept of the partition rule is introduced for analyzing the influences of the reactions of outside players on the deviating coalition in sequencing situations. Furthermore, the cores of partition sequencing games are defined based on the partition rules and we present some relationships between the EGS rule and these cores. Section 4.4 provides a mechanism for implementing the EGS rule. 


\subsection{Partition sequencing games}

In this section, we consider sequencing situations in which externalities are involved. First, an informal description of our model: Consider the case where some players in the queue intend to deviate and form a coalition $S$. Once formed, the initial agreement of the grand coalition is torn up, and the players in that coalition are free to reorganize themselves within $S$. What happens after the deviation is that all of the remaining players are also allowed to form coalitions freely, and settle on the rearrangement of positions within each coalition, as the initial agreement of the grand coalition no longer exists. Then a partition of $N$ is naturally generated, and every coalition contains several "groups" which are their $\sigma_{0}$-components. Next, we assume that groups can negotiate with each other so they can decide whether or not to trade places with a neighbor group. In this chapter, when we say that two neighbor groups are involved in a negotiation, we mean that they are predicting the outcome of exchanging their group positions. If the cost changes generated by a switch between neighbor groups are positive, then the two groups will exchange their positions and the ensuing benefits will be equally shared by them. Every group continues this process until there is no group able to win more surplus. The final worth of a coalition under this circumstance not only relies on the coalition itself but also on the reactions of the non-members. For better illustrating this process, it is more convenient to consider the following example first.

Example 4.1. Let $\left(N, \sigma_{0}, w, p\right)$ be a sequencing situation, where $N=\{1,2,3,4\}$, $\sigma_{0}=(1,2,3,4), w=(1,1,1,1)$ and $p=(3,2,3,1)$.

In this case, assume that players 1 and 2 contemplate forming a coalition to decrease their total costs. Once formed, they will attain 1 cost savings by internal collaboration-switching their positions. In this example, Curiel et al. [21] take the perspective that once coalition $\{1,2\}$ forms, no external factor is taken into account and the worth of that coalition equals to the maximal cost savings obtained by their internal collaboration, which is 1. From a practical point of view, it is quite conceivable that there are mutual influences among coalitions in scheduling problems. 
Now let us take into account the influences from external players. Two different cases may occur when coalition $\{1,2\}$ forms: (i) 3 and 4 form another coalition $\{3,4\}$, or (ii) 3 and 4 are unwilling to cooperate and form $\{3\}$, $\{4\}$. So there are two possible partitions for $N$, which are $\{\{1,2\},\{3,4\}\}$, and $\{\{1,2\},\{3\},\{4\}\}$. Different results can lead up to different worths of coalition $\{1,2\}$ if we see the actions of trading places between coalitions as the main source of externalities. If coalition $\{1,2\}$ predicts that the outside members will form $\{3,4\}$, besides the benefit from the internal collaboration, $\{1,2\}$ can guarantee itself more income if it asks to negotiate with $\{3,4\}$ for trading places of coalitions. To see why, if the two coalitions switch their places of coalitions, that is, the sequencing order changes from $(2,1,4,3)$ to $(4,3,2,1)$, the cost savings obtained by this rearrangement equal 2 , which will be shared by the two coalitions. The final worth of coalition $\{1,2\}$ in the situation described above is 2 if the cost savings from the negotiation between coalitions are equally shared. If coalition $\{1,2\}$ predicts that the outside members will form $\{3\},\{4\}$, then $\{1,2\}$ can first wait for $\{3\}$ and $\{4\}$ to finish their switch (which will generate 2 units of cost savings for $\{3\}$ and $\{4\})$ and then trade places with coalition $\{4\}$ to obtain half of the cost savings, which are 1.5. Then the final worth of $\{1,2\}$, in this case, is 2.5. ${ }^{1}$

Example 4.1 provides a special case that both the deviating coalition and later-formed coalitions are connected. However, in general, the coalitions under consideration can be disconnected. Before we give a formal definition of the sequencing games where externalities exist, the following divisions have to be considered.

Given a sequencing situation $\left(N, \sigma_{0}, w, p\right)$, suppose that the deviation of a coalition $S$ has triggered a partition $\rho$ of $N$, where $S \in \rho$. Then the division $M$ of $N$, derived from $\left(N, \sigma_{0}, w, p\right)$ and $\rho$, is a partition composed of $\sigma_{0}$-components of all coalitions in $\rho .^{2}$ Every element in $M$ is called a group.

\footnotetext{
${ }^{1}$ The reason why $\{1,2\}$ does not switch its position with $\{3\}$ is that they will get negative cost changes.

${ }^{2}$ Strictly speaking, the symbol $M$ should be $M_{\rho}^{\sigma_{0}}$, since a division is dependent on the corresponding initial order and partition. But for notation simplicity, in this chapter, we write $M$ instead of $M_{\rho}^{\sigma_{0}}$.
} 
Obviously, every connected coalition in $\rho$ forms a group by itself. We can notice that $\rho=M$ if and only if every coalition in $\rho$ is connected. The following example helps understand the notion of division.

Example 4.2. Let $\left(N, \sigma_{0}, w, p\right)$ be a sequencing situation and $\rho$ a partition of $N$, where $N=\{1,2,3,4,5,6\}, \sigma_{0}=(1,2,3,4,5,6)$ and $\rho=\{\{1,3,4\},\{2,5,6\}\}$. Then the division derived from $\left(N, \sigma_{0}, w, p\right)$ and $\rho$ is $M=\{\{1\},\{2\},\{3,4\},\{5,6\}\}$.

Analogous to the order on the players, we introduce the concept of the group order, which is a bijection $\pi: M \rightarrow|M|$, where $\pi(T)=j$ means that $T$ is on the $j$-th group position in the queue. The set of predecessors of group $T \in$ $M$ is denoted by $P(\pi, T)=\{J \in M \mid \pi(J)<\pi(T)\}$ and the set of successors of the group $T \in M$ by $F(\pi, T)=\{J \in M \mid \pi(J)>\pi(T)\}$.

We now formally provide the definition of the corresponding partition sequencing game, ${ }^{3}$ in which the worth of a coalition relies on a two-stage process prescribed in the following:

Stage 1 (Internal collaboration stage):

At this stage, a coalition $S \subseteq N$ decides to split away from the grand coalition and work alone. The members within $S$ can ensure themselves the maximal cost savings by rearranging their positions in the coalition. The surplus of any coalition $S$ at this stage is defined by

$$
E_{1}(S)=\max _{\sigma \in \Sigma_{S}} \sum_{i \in S} w_{i}\left(C\left(\sigma_{0}, i\right)-C(\sigma, i)\right) .
$$

Since the WSPT rule is the optimal order to obtain the maximal cost savings for the grand coalition, we can also conclude that for any connected coalition $T \subseteq N$, the optimal arrangement is to have the players of $T$ reordered in non-increasing urgency indices. Then it holds that for any connected coalition $T$

$$
E_{1}(T)=\sum_{\substack{i, j \in T \\ \sigma_{0}(i)<\sigma_{0}(j)}} g_{i j}
$$

\footnotetext{
${ }^{3}$ In [30] they introduce the so-called "partitioning sequencing game" arising from the situation in which jobs arrive in batches and the jobs that arrive earlier have some privileges over jobs in later arriving batches. What should be noted is that, despite an analogous name, our game is different essentially from theirs.
} 
For any coalition $S$ that is not connected with respect to $\sigma_{0}$, we have

$$
E_{1}(S)=\sum_{T \in S / \sigma_{0}} E_{1}(T)
$$

The first stage surplus function $E_{1}: 2^{N} \rightarrow \mathbb{R}$, defined in (4.1), coincides with the characteristic function in sequencing games introduced by Curiel et al. [21]. However, besides the point of internal collaboration which has already been concerned in [21], the partition sequencing games that we present in this chapter will contain the following second stage.

Stage 2 (External negotiation stage):

Suppose that the cooperative behavior of coalition $S$ triggers a partition $\rho$ of $N$, where $S \in \rho$, and those later-formed coalitions have also collected their maximal cost savings by internal collaborations which are the same as at stage 1. After ensuring the benefits from the first stage, every coalition in $\rho$ now has to consider negotiating with other coalitions for gaining more surplus. Before the discussion on the definition of the surplus that a coalition will obtain during this stage, it is necessary as a starting point to think about "how much benefit will a group of a coalition get".

Let $M$ be the division derived from $\left(N, \sigma_{0}, w, p\right)$ and $\rho$, and let $\pi$ be the corresponding group order. Inspired by Example 4.2, we assume that, in this stage, all groups in $M$ are free to negotiate with each other for trading their group positions. The negotiation here means that every two neighbor groups have to decide whether to exchange their group positions, depending on their predictions of the cost changes after the implementation. For any two groups $I, J \in M$ with $\pi(I)=\pi(J)-1$, denote the cost changes generated by the switch of $I, J$ by $G_{I J}^{*}$. In this chapter, we assume that the relative positions of players in each group do not change during the group switch process. Suppose that group $J$ contains $k$ members, i.e., $J=\left\{j_{1}, \cdots, j_{k}\right\}$. 
Then it is easy to verify that

$$
\begin{aligned}
G_{I J}^{*} & =\sum_{i \in I} p_{i} \sum_{j \in J} w_{j}-\sum_{j \in J} p_{j} \sum_{i \in I} w_{i} \\
& =\sum_{i \in I} p_{i}\left(w_{j_{1}}+\cdots+w_{j_{k}}\right)-\sum_{i \in I} w_{i}\left(p_{j_{1}}+\cdots+p_{j_{k}}\right) \\
& =\sum_{i \in I}\left[\left(p_{i} w_{j_{1}}-w_{i} p_{j_{1}}\right)+\cdots+\left(p_{i} w_{j_{k}}-w_{i} p_{j_{k}}\right)\right] \\
& =\sum_{i \in I} \sum_{j \in J}\left(w_{j} p_{i}-w_{i} p_{j}\right) \\
& =\sum_{i \in I} \sum_{j \in J} g_{i j}^{*} .
\end{aligned}
$$

Define the gain of the negotiation between $I$ and $J$ as

$$
G_{I J}=\max \left\{G_{I J}^{*}, 0\right\}
$$

If $G_{I J}^{*}>0$, the negotiation becomes successful and the two groups switch their group positions. Otherwise, the negotiation breaks, and the two groups stay where they are. The gain of the negotiation between the two groups defined here is similar to the gain between players. In fact, if we define the composed cost coefficient, the composed processing time and the composed urgency index for a coalition $S \subseteq N$ by, respectively, $w_{S}=\sum_{i \in S} w_{i}, p_{S}=\sum_{i \in S} p_{i}$ and $u_{S}=$ $\frac{w_{S}}{p_{S}}$, then it is readily to see that $G_{I J}^{*}=w_{J} p_{I}-w_{I} p_{J}$, and $G_{I J}^{*}>0$ if and only if $u_{J}>u_{I}$. In this stage, all groups continue to negotiate with their new neighbors until there is no group able to win more surplus by switching. It can be easily seen that the final group order, in which no group is willing to exchange its group position with others, can be obtained by arranging all groups according to non-increasing composed urgency indices.

Note that by the definition of divisions, no two neighbor groups belong to the same coalition at the beginning of stage 2. However, two groups from the same coalition may become neighbors at some point during the process of switching. When that happens, according to the model established above, we implicitly assume that the two groups have their own autonomy and rule out the possibility of the merge between them. The intuition behind this 
tacit assumption is that every group of a coalition is allowed to have its own contract. This feature is present in a multitude of economic situations where the enterprises are given the autonomous right in corporation alliances.

Inspired by the idea of allocation contained in the EGS rule in [21], we assume that the gain of a negotiation is equally divided between the two groups. Thus for any group $T \in M$, the surplus of $T$ at the second stage is defined by

$$
E_{2}(T ; M)=\frac{1}{2} \sum_{K \in P(\pi, T)} G_{K T}+\frac{1}{2} \sum_{L \in F(\pi, T)} G_{T L} .
$$

Then, we define the surplus of $S$ in the second stage by the sum of the surpluses of the $\sigma_{0}$-components of $S$, i.e.,

$$
E_{2}(S ; \rho)=\sum_{T \in S / \sigma_{0}} E_{2}(T ; M) .
$$

Definition 4.1. Let $\left(N, \sigma_{0}, w, p\right)$ be a sequencing situation. The corresponding partition sequencing game $(N, v)$ is defined by

$$
v(S ; \rho)=E_{1}(S)+E_{2}(S ; \rho),
$$

where $S \in \rho, E_{1}(S)$ and $E_{2}(S ; \rho)$ are defined as in (4.1) and (4.3).

The partition sequencing games are in fact a class of PFF games. The worth of a coalition is defined as the sum of the surpluses generated from the two-stage process described above. We can clearly see that although the surplus generated from the first stage is a constant value, the surplus generated from the second stage is conditioned on the partition of external players.

It should be noted that when the coalition under consideration is the grand coalition, the worth $v(N ;\{N\})$ must be equal to $\sum_{i, j \in N: \sigma_{0}(i)<\sigma_{0}(j)} g_{i j}$ since there is no external player and only stage 1 is involved. In this sense, the definition of the total surplus needing to be shared is consistent with much of the literature in which the sequencing games are in CFF. Furthermore, observe that the idea of EGS rule is put forward in the second stage, one can easily verify that if the division $M$ of $N$, derived from $\left(N, \sigma_{0}, w, p\right)$ and $\rho$, is formed only of singletons, the surplus that any group $\{i\}$ can obtain in this 
stage coincides with $E G S_{i}\left(N, \sigma_{0}, w, p\right)$. If this is the case, then we can immediately have $v(S ; \rho)=\sum_{i \in S} E G S_{i}\left(N, \sigma_{0}, w, p\right)$ for any $S \in \rho$.

Before we present our main results, we first need to look at the relationship between the gain of the switch of two neighbor groups and the gains between their members. Let's consider the following example.

Example 4.3. In Example 4.2, set $w=(1,5,3,4,2,6), p=(1,1,1,1,1,1)$. The gain of the negotiation between the groups $\{3,4\}$ and $\{5,6\}$ is $G_{\{3,4\}\{5,6\}}=2$. The sum of the gains between their members is $\sum_{i \in\{3,4\}} \sum_{j \in\{5,6\}} g_{i j}=5$. Hence, we have $G_{\{3,4\}\{5,6\}}<\sum_{i \in\{3,4\}} \sum_{j \in\{5,6\}} g_{i j}$.

Example 4.3 provides a special case showing that the gain of the switch of two neighbor groups is not greater than the sum of gains between their members. This makes us wonder if the result is a general conclusion. The answer is affirmative, which can be shown by the following lemma.

Lemma 4.1. Let $\left(N, \sigma_{0}, w, p\right)$ be a sequencing situation, $\rho$ a partition of $N$, and $M$ the corresponding division. Then for any $I, J \in M$, it holds that

$$
G_{I J} \leq \sum_{i \in I} \sum_{j \in J} g_{i j}
$$

Proof. Because $G_{I J}=\max \left\{G_{I J}^{*}, 0\right\}$, it follows from (4.2) that

$$
G_{I J}=\max \left\{\sum_{i \in I} \sum_{j \in J} g_{i j}^{*}, 0\right\} \leq \sum_{i \in I} \sum_{j \in J} \max \left\{g_{i j}^{*}, 0\right\}=\sum_{i \in I} \sum_{j \in J} g_{i j} .
$$

Now another question arises: under what conditions does the equality in Lemma 4.1 hold? To answer this question, it's necessary for us to introduce a new concept, which is called the simple group. In general, a simple group is a connected group with respect to $\hat{\sigma}$, where $\hat{\sigma}$ is an optimal order with respect to $N$. To put it formally, let $M$ be a division derived from a sequencing situation $\left(N, \sigma_{0}, w, p\right)$ and a partition $\rho$ of $N$, and let $\hat{\sigma}$ be an optimal order with respect to $N$. A group $T \in M$ is called simple with respect to $\hat{\sigma}$ if (i) $|T|=$ 1 , or (ii) $|T| \geq 2$ and for any $i, j \in T$ with $\hat{\sigma}(i)<\hat{\sigma}(j)$, there does not exist 
a $k \in N \backslash T$ such that $\hat{\sigma}(i)<\hat{\sigma}(k)<\hat{\sigma}(j)$. For instance, in Example 4.3, the unique optimal order is $\hat{\sigma}=(6,2,4,3,5,1)$. The groups $\{1\},\{2\}$, and $\{3,4\}$ are simple groups with respect to $\hat{\sigma}$ but $\{5,6\}$ is not. The following lemma shows that the equality in Lemma 4.1 holds if and only if there exists an optimal order such that all groups are simple.

Lemma 4.2. Let $\left(N, \sigma_{0}, w, p\right)$ be a sequencing situation, $\rho$ a partition of $N$, and $M$ the corresponding division. For any $I, J \in M$,

$$
G_{I J}=\sum_{i \in I} \sum_{j \in J} g_{i j}
$$

if and only if there exists an optimal order $\hat{\sigma}$ such that all groups in $M$ are simple with respect to $\hat{\sigma}$.

Proof. For any groups $I, J \in M$, we define $\underline{i}=\arg \min _{i \in I} u_{i}, \bar{i}=\arg \max _{i \in I} u_{i}$, $\underline{j}=\arg \min _{j \in J} u_{j}$, and $\bar{j}=\arg \max _{j \in J} u_{j}$. From the proof of Lemma 4.1, it is easy to see that for any $I, J \in M, G_{I J}=\sum_{i \in I} \sum_{j \in J} g_{i j}$ if and only if for any $I, J \in M$, either $u_{\underline{i}} \geq u_{\bar{j}}$ or $u_{\underline{j}} \geq u_{\bar{i}}$. For notation simplicity, we call two groups $I, J$ comparable if $u_{\underline{i}} \geq u_{\bar{j}}$ or $u_{j} \geq u_{\bar{i}}$. To complete the proof, we only have to show that for any $I, J \in M$, the groups $I, J$ are comparable if and only if there exists an optimal order $\hat{\sigma}$ such that all groups in $M$ are simple with respect to $\hat{\sigma}$.

(If). We prove this part by absurdity. Suppose that there are two groups $I, J$ which are not comparable. Then $u_{\underline{i}}<u_{\bar{j}}$ and $u_{\underline{j}}<u_{\bar{i}}$.

Case 1: $u_{\underline{i}}>u_{j}$. Then $u_{j}<u_{\underline{i}}<u_{\bar{j}}$. It follows that for any optimal order $\hat{\sigma}$ we have $\hat{\sigma}(\bar{j})<\hat{\sigma}(\underline{i})<\hat{\sigma}(\underline{j})$. Hence, $J$ can not be a simple group with respect to any optimal order, a contradiction.

Case 2: $u_{\bar{i}}>u_{\bar{j}}$. Then $u_{\underline{i}}<u_{\bar{j}}<u_{\bar{i}}$. It follows that for any optimal order $\hat{\sigma}$ we have $\hat{\sigma}(\bar{i})<\hat{\sigma}(\bar{j})<\hat{\sigma}(\underline{i})$. Hence, $I$ can not be a simple group with respect to any optimal order, a contradiction.

Case 3: $u_{\underline{i}} \leq u_{j}$ and $u_{\bar{i}} \leq u_{\bar{j}}$. We have $u_{\underline{i}} \leq u_{j}<u_{\bar{i}} \leq u_{\bar{j}}$. Then for any optimal order $\hat{\sigma}$, there are four possibilities: $\hat{\sigma}(\bar{j})<\hat{\sigma}(\bar{i})<\hat{\sigma}(j)<\hat{\sigma}(\underline{i})$ or $\hat{\sigma}(\bar{i})<\hat{\sigma}(\bar{j})<\hat{\sigma}(\underline{j})<\hat{\sigma}(\underline{i})$ or $\hat{\sigma}(\bar{j})<\hat{\sigma}(\bar{i})<\hat{\sigma}(\underline{i})<\hat{\sigma}(\underline{j})$ or $\hat{\sigma}(\bar{i})<\hat{\sigma}(\bar{j})<$ 
$\hat{\sigma}(\underline{i})<\hat{\sigma}(j)$. So for any optimal order, there is at least one of the two groups is not simple with respect to $\hat{\sigma}$, a contradiction.

(Only if). If for any $I, J \in M$, the groups $I, J$ are comparable, then there must exist a sequence of groups $\left(I_{1}, \ldots, I_{m}\right)$ such that for any $k<l$, $\min _{i \in I_{l}} u_{i} \geq \max _{j \in I_{k}} u_{j}$. Let $n_{k}$ be the cardinal number of the group $I_{k}$ and the sequence $\left(i_{k}^{1}, \ldots, i_{k}^{n_{k}}\right)$ the order of players in $I_{k}$ with respect to the WSPT rule. It follows immediately that the order $\sigma=\left(i_{1}^{1}, \ldots, i_{1}^{n_{1}}, \ldots, i_{m}^{1}, \ldots, i_{m}^{n_{m}}\right)$ is an optimal order and all groups in $M$ are simple with respect to $\sigma$.

By Lemma 4.1, we can prove the following result, which shows that partition sequencing games are cohesive.

Theorem 4.3. Let $\left(N, \sigma_{0}, w, p\right)$ be a sequencing situation and $(N, v)$ the corresponding partition sequencing game. Then $(N, v)$ is cohesive.

Proof. We have already mentioned that $v(N ;\{N\})=\sum_{i, j \in N: \sigma_{0}(i)<\sigma_{0}(j)} g_{i j}$. We now only have to show that $\sum_{S \in \rho} v(S ; \rho) \leq \sum_{i, j \in N: \sigma_{0}(i)<\sigma_{0}(j)} g_{i j}$. For any partition $\rho$ of $N$, let $M$ be the division derived from $\left(N, \sigma_{0}, w, p\right)$ and $\rho$, and $\pi$ the corresponding group order. Then

$$
\begin{aligned}
\sum_{S \in \rho} v(S ; \rho) & =\sum_{S \in \rho} E_{1}(S)+\sum_{S \in \rho} E_{2}(S ; \rho) \\
& =\sum_{S \in \rho} \sum_{T \in S / \sigma_{0}} E_{1}(T)+\sum_{S \in \rho} \sum_{T \in S / \sigma_{0}} E_{2}(T ; M) \\
& =\sum_{T \in M} \sum_{\substack{i, j \in T \\
\sigma_{0}(i)<\sigma_{0}(j)}} g_{i j}+\sum_{T \in M} E_{2}(T ; M) \\
& =\sum_{T \in M} \sum_{\substack{i, j \in T \\
\sigma_{0}(i)<\sigma_{0}(j)}} g_{i j}+\sum_{\substack{I, J \in M \\
\pi(I)<\pi(J)}} G_{I J} \\
& \leq \sum_{T \in M} \sum_{\substack{i, j \in T \\
\sigma_{0}(i)<\sigma_{0}(j)}} g_{i j}+\sum_{\substack{I, J \in M \\
\pi(I)<\pi(J)}} \sum_{i \in I} g_{i j} \\
& =\sum_{\substack{i, j \in N \\
\sigma_{0}(i)<\sigma_{0}(j)}} g_{i j},
\end{aligned}
$$


where the second equality follows from the definitions of $E_{1}(S)$ and $E_{2}(S ; \rho)$, and the inequality follows from Lemma 4.1.

The intuition behind Theorem 4.3 is straightforward: the arrangement in the grand coalition is the most effective way to reach the maximal cost savings, while other coalition structures impose constraints on the range of possible reorganizations of players. However, the following result shows that the grand coalition may not be the only coalition structure leading to the maximal total surplus, the proof of which follows immediately from Theorem 4.3 and Lemma 4.2 .

Corollary 4.1. Let $\left(N, \sigma_{0}, w, p\right)$ be a sequencing situation, $(N, v)$ the corresponding partition sequencing game, $\rho$ a partition of $N$ and $M$ the corresponding division. Then

$$
v(N ;\{N\})=\sum_{S \in \rho} v(S ; \rho)
$$

if and only if there exists an optimal order $\hat{\sigma}$ such that all groups in $M$ are simple with respect to $\hat{\sigma}$.

In PFF games, the side effects of forming coalitions on third parties have always been the main concerns in the literature. It turns out that the merger of external coalitions does not have a positive effect on the deviating players in partition sequencing games as Theorem 4.4 shows.

Theorem 4.4. Let $\left(N, \sigma_{0}, w, p\right)$ be a sequencing situation. The corresponding partition sequencing game has non-positive externalities.

Proof. We need to show that for any mutually disjoint coalitions $S_{1}, S_{2}, S_{3} \subseteq$ $N$, and for any partition $\rho^{\prime}$ of $N \backslash\left(S_{1} \cup S_{2} \cup S_{3}\right)$,

$$
v\left(S_{1} ;\left\{S_{1}, S_{2} \cup S_{3}\right\} \cup \rho^{\prime}\right) \leq v\left(S_{1} ;\left\{S_{1}, S_{2}, S_{3}\right\} \cup \rho^{\prime}\right) .
$$

Given any $\left(N, \sigma_{0}, w, p\right)$ and $\rho$ of $N$, let $M$ and $\pi$ be the corresponding division and group order respectively. From the definition of the partition 
sequencing games, we have

$$
\begin{aligned}
& v\left(S_{1} ; \rho\right)=\sum_{T \in S_{1} / \sigma_{0}} E_{1}(T)+\sum_{T \in S_{1} / \sigma_{0}} E_{2}(T ; M) \\
& =\sum_{T \in S_{1} / \sigma_{0}} \sum_{\substack{i, j \in T \\
\sigma_{0}(i)<\sigma_{0}(j)}} g_{i j} \\
& +\frac{1}{2} \sum_{T \in S_{1} / \sigma_{0}}\left(\sum_{K \in P(\pi, T)} G_{K T}+\sum_{L \in F(\pi, T)} G_{T L}\right) \\
& =\sum_{T \in S_{1} / \sigma_{0}} \sum_{\substack{i, j \in T \\
\sigma_{0}(i)<\sigma_{0}(j)}} g_{i j}+\sum_{\substack{I, J \in S_{1} / \sigma_{0} \\
\pi(I)<\pi(J)}} G_{I J} \\
& +\frac{1}{2} \sum_{T \in S_{1} / \sigma_{0}}\left(\sum_{\substack{K \in P(\pi, T) \\
K \notin S_{1} / \sigma_{0}}} G_{K T}+\sum_{\substack{L \in F(\pi, T) \\
L \notin S_{1} / \sigma_{0}}} G_{T L}\right) .
\end{aligned}
$$

Let $\rho_{1}=\left\{S_{1}, S_{2} \cup S_{3}\right\} \cup \rho^{\prime}$ and $\rho_{2}=\left\{S_{1}, S_{2}, S_{3}\right\} \cup \rho^{\prime}$. Denote the corresponding divisions and group orders derived from $\left(N, \sigma_{0}, w, p\right), \rho_{1}$ and $\left(N, \sigma_{0}, w, p\right), \rho_{2}$ respectively by $M_{1}, \pi_{1}$ and $M_{2}, \pi_{2}$. That is, $M_{1}, \pi_{1}$ are the division and group order after the merge of $S_{2}$ and $S_{3}$, and $M_{2}, \pi_{2}$ are the division and group order before the merge. Note that in (4.6), the terms $\sum_{T \in S / \sigma_{0}} \sum_{i, j \in T: \sigma_{0}(i)<\sigma_{0}(j)} g_{i j}$ and $\sum_{I, J \in S / \sigma_{0}: \pi(I)<\pi(J)} G_{I J}$ are independent of the coalition structure of external players. Hence, in order to prove (4.5) we only have to show that for any $T \in S_{1} / \sigma_{0}$,

$$
\sum_{\substack{K \in P\left(\pi_{1}, T\right) \\ K \notin S_{1} / \sigma_{0}}} G_{K T}+\sum_{\substack{L \in F\left(\pi_{1}, T\right) \\ L \notin S_{1} / \sigma_{0}}} G_{T L} \leq \sum_{\substack{K \in P\left(\pi_{2}, T\right) \\ K \notin S_{1} / \sigma_{0}}} G_{K T}+\sum_{\substack{L \in F\left(\pi_{2}, T\right) \\ L \notin S_{1} / \sigma_{0}}} G_{T L} \cdot
$$

This can be done if we are able to prove

$$
\sum_{\substack{K \in P\left(\pi_{1}, T\right) \\ K \notin S_{1} / \sigma_{0}}} G_{K T} \leq \sum_{\substack{K \in P\left(\pi_{2}, T\right) \\ K \notin S_{1} / \sigma_{0}}} G_{K T}
$$


and

$$
\sum_{\substack{L \in F\left(\pi_{1}, T\right) \\ L \notin S_{1} / \sigma_{0}}} G_{T L} \leq \sum_{\substack{L \in F\left(\pi_{2}, T\right) \\ L \notin S_{1} / \sigma_{0}}} G_{T L}
$$

separately.

Next, we only show that (4.7) holds, since the proof of (4.8) is almost identical to that of (4.7). Since $S_{2}$ and $S_{3}$ are disjoint, it is easy to see that for any $K \in M_{1}$, there are groups $K_{1}, \ldots, K_{k}$ in $M_{2}$ such that $K=\bigcup_{i=1}^{k} K_{i}$. Let $M_{1}^{*}$ be the set of groups in $M_{1}$ that contain both the members of $S_{2}$ and the members of $S_{3}$, i.e., $M_{1}^{*}=\left\{J \in M_{1} \mid J \cap S_{2} \neq \emptyset\right.$ and $\left.J \cap S_{3} \neq \emptyset\right\}$. Obviously, $k=1$ if and only if $K \in M_{1} \backslash M_{1}^{*}$.

Let $K \in M_{1}$ and $T \in M_{1} \backslash M_{1}^{*}$ with $\pi_{1}(K)<\pi_{1}(T)$. Then

$$
\begin{aligned}
G_{K T} & =\max \left\{\sum_{i \in K} \sum_{j \in T} g_{i j}^{*}, 0\right\} \\
& =\max \left\{\sum_{h=1}^{k} \sum_{i \in K_{h}} \sum_{j \in T} g_{i j}^{*}, 0\right\} \\
& =\max \left\{\sum_{h=1}^{k} G_{K_{h} T}^{*}, 0\right\} \\
& \leq \sum_{h=1}^{k} \max \left\{G_{K_{h} T}^{*}, 0\right\} \\
& =\sum_{h=1}^{k} G_{K_{h} T},
\end{aligned}
$$


where the first equality and the third equality follow from (4.2). Note that any $T \in S_{1} / \sigma_{0}$ is a group in $M_{1} \backslash M_{1}^{*}$. Hence, for any $T \in S_{1} / \sigma_{0}$,

$$
\begin{aligned}
\sum_{\substack{K \in P\left(\pi_{1}, T\right) \\
K \notin S_{1} / \sigma_{0}}} G_{K T} & =\sum_{\substack{K \in P\left(\pi_{1}, T\right) \\
K \notin S_{1} / \sigma_{0} \\
K \in M_{1} \backslash M_{1}^{*}}} G_{K T}+\sum_{\substack{K \in P\left(\pi_{1}, T\right) \\
K \notin S_{1} / \sigma_{0} \\
K \in M_{1}^{*}}} G_{K T} \\
& \leq \sum_{\substack{K \in P\left(\pi_{1}, T\right) \\
K \notin S_{1} / \sigma_{0} \\
K \in M_{1} \backslash M_{1}^{*}}} G_{K T}+\sum_{\substack{K \in P\left(\pi_{1}, T\right) \\
K \notin S_{1} / \sigma_{0} \\
K \in M_{1}^{*}}} \sum_{h=1}^{k} G_{K_{h} T} \\
& =\sum_{\substack{K \in P\left(\pi_{2}, T\right) \\
K \notin S_{1} / \sigma_{0}}} G_{K T},
\end{aligned}
$$

which proves (4.7). Here the inequality follows from (4.9) and the last equality follows from the fact that any group in $M_{1} \backslash M_{1}^{*}$ is also a group in $M_{2}$.

Hafalir [36] provides a natural extension of convexity to a PFF game: a PFF game is convex if for any $S, T \subseteq N$ and any partition $\rho$ of $N \backslash(S \cup T)$,

$$
\begin{aligned}
v(S \cup T ;\{S \cup T\} \cup \rho) & +v(S \cap T ;\{S \cap T, S \backslash T, T \backslash S\} \cup \rho) \\
& \geq v(S ;\{S, T \backslash S\} \cup \rho)+v(T ;\{T, S \backslash T\} \cup \rho) .
\end{aligned}
$$

Hafalir showed that convex PFF games have many nice properties. For example, the convexity of a PFF game implies the cohesiveness and the nonemptiness of the core with singleton expectations of external players. However, we will show by our next example that the partition sequencing games do not necessarily satisfy this convexity.

Example 4.4. Let $\left(N, \sigma_{0}, w, p\right)$ be a sequencing situation, where $N=\{1,2,3,4\}$, $w=(2,1,4,3), p=(1,1,1,1), \sigma_{0}=(1,2,3,4)$. Let $\rho$ be the partition of $N$ with $\rho=\{\{1\},\{2\},\{3,4\}\}$.

It can easily be calculated that $v(\{2\} ; \rho)=2.5, v(\{3,4\} ; \rho)=4$, and $v(\{2,3,4\} ;\{\{1\},\{2,3,4\}\})=6$. Then

$$
v(\{2,3,4\} ;\{\{1\},\{2,3,4\}\})<v(\{2\} ; \rho)+v(\{3,4\} ; \rho) .
$$


Hence, this partition sequencing game is not convex. As a side result, Example 4.4 also indicates that partition sequencing games do not necessarily have superadditivity. Naturally, the superadditivity of PFF games can be extended from that of CFF games: a PFF is superadditive if for any $S, T \subseteq N$ with $S \cap T=\emptyset$, and any partition $\rho$ of $N \backslash(S \cup T), v(S \cup T ;\{S \cup T\} \cup \rho) \geq v(S ;\{S, T\} \cup \rho)+$ $v(T ;\{S, T\} \cup \rho)$.

\subsection{Partition rules and the core}

\subsubsection{Partition rules}

A partition rule is a mapping $R: 2^{N} \rightarrow \Pi_{N}$. That is, for every coalition $S$ which wants to deviate from $N$, there is a predetermined partition $R(S) \in \Pi_{N}$ for $S$, where $S \in R(S)$. Thus, the worth $v(S ; R(S))$ of any coalition is made certain as soon as the partition rule $R$ is determined. A partition rule can be thought of as a mandatory regulation restricting the behavior of external players when some coalition deviates. Alternatively, it can be considered as the expectation of deviating coalition for the others' reactions. We denote by $\mathscr{R}$ the set of all partition rules. In the following, we provide two different partition rules ${ }^{4}$ that have already been mentioned in the literature.

$m$-Exogenous rule: $\mathrm{m}$-Exogenous rule $R^{m}$ requires all external players to join in a single coalition $N \backslash S[36,56]$. Formally,

$$
R^{m}(S)=\{S, N \backslash S\}
$$

$s$-Exogenous rule: $s$-Exogenous rule $R^{s}$ requires all external players to form singletons, which means that the partition of $N \backslash S$ must be $[N \backslash S][24,36]$. Formally,

$$
R^{S}(S)=\{S,[N \backslash S]\}
$$

In partition sequencing games, if a coalition $S \subset N$ in the queue decides to deviate, the final surplus of $S$ depends on the reactions of external players.

\footnotetext{
${ }^{4}$ The partition rules we provide here are analogous to some of the expectation formation rules defined by Bloch and Van den Nouweland [8].
} 
Faced with this fact, a natural question is: which kind of partition should external players choose to form in such a way that the deviating coalition $S$ will receive the maximal payoff or the minimal payoff? The answer, which can be immediately deduced from Theorem 4.4, is that the partition of $N \backslash S$ best for $S$ coincides with $[N \backslash S]$ and the worst for $S$ coincides with $\{N \backslash S\}$. Therefore, in sequencing situations, the m-exogenous rule, and s-exogenous rule will sometimes be called the optimistic rule and pessimistic rule respectively.

\subsubsection{Cores of partition sequencing games}

We next define the cores of partition sequencing games, which depend on the partition rules applied to the corresponding sequencing situations.

Definition 4.2. Let $\left(N, \sigma_{0}, w, p\right)$ be a sequencing situation. The core $C_{R}(v)$ of the corresponding partition sequencing game $(N, v)$ with respect to partition rule $R \in \mathscr{R}$ is the set of vectors $x=\left(x_{1}, x_{2}, \ldots, x_{n}\right) \in \mathbb{R}^{n}$ satisfying the following conditions:

(1) $\sum_{i \in N} x_{i}=v(N ;\{N\})$;

(2) $\sum_{i \in S} x_{i} \geq v(S ; R(S))$ for any $S \subseteq N$.

The next theorem says that the EGS rule always yields a core element no matter which partition rule is applied to the game.

Theorem 4.5. Let $\left(N, \sigma_{0}, w, p\right)$ be a sequencing situation and $(N, v)$ the corresponding partition sequencing game. Then we have for any partition rule $R \in \mathscr{R}$, the corresponding core $C_{R}(v) \neq \emptyset$ and $E G S\left(N, \sigma_{0}, w, p\right) \in C_{R}(v)$.

Proof. It is easy to verify that

$$
v(N ;\{N\})=\sum_{i \in N} E G S_{i}\left(N, \sigma_{0}, w, p\right) .
$$

We only have to show that for any coalition $S \subset N$ and any partition rule $R \in \mathscr{R}$,

$$
v(S ; R(S)) \leq \sum_{i \in S} E G S_{i}\left(N, \sigma_{0}, w, p\right) .
$$


Given any $S \subset N$ and any partition rule $R \in \mathscr{R}$, let $M$ and $\pi$ be the division and group order derived from $\left(N, \sigma_{0}, w, p\right)$ and $R(S)$. Then

$$
\begin{aligned}
& v(S ; R(S))=\sum_{T \in S / \sigma_{0}} E_{1}(T)+\sum_{T \in S / \sigma_{0}} E_{2}(T ; M) \\
& =\sum_{T \in S / \sigma_{0}} \sum_{\substack{i, j \in T \\
\sigma_{0}(i)<\sigma_{0}(j)}} g_{i j}+\sum_{\substack{I, J \in S / \sigma_{0} \\
\pi(I)<\pi(J)}} G_{I J} \\
& +\frac{1}{2} \sum_{T \in S / \sigma_{0}}\left(\sum_{\substack{K \in P(\pi, T) \\
K \notin S / \sigma_{0}}} G_{K T}+\sum_{\substack{L \in F(\pi, T) \\
L \notin S / \sigma_{0}}} G_{T L}\right) \\
& \leq \sum_{T \in S / \sigma_{0}} \sum_{\substack{i, j \in T \\
\sigma_{0}(i)<\sigma_{0}(j)}} g_{i j}+\sum_{\substack{I, J \in S / \sigma_{0} \\
\pi(I)<\pi(J)}} \sum_{i \in I} \sum_{j \in J} g_{i j} \\
& +\frac{1}{2} \sum_{T \in S / \sigma_{0}}\left(\sum_{\substack{K \in P(\pi, T) \\
K \notin S / \sigma_{0}}} \sum_{k \in K} \sum_{i \in T} g_{k i}+\sum_{\substack{L \in F(\pi, T) \\
L \notin S / \sigma_{0}}} \sum_{i \in T} \sum_{l \in L} g_{i l}\right) \\
& =\sum_{i \in S} \frac{1}{2}\left(\sum_{k \in P\left(\sigma_{0}, i\right)} g_{k i}+\sum_{l \in F\left(\sigma_{0}, i\right)} g_{i l}\right) \\
& =\sum_{i \in S} E G S_{i}\left(N, \sigma_{0}, w, p\right) \text {, }
\end{aligned}
$$

where the first equality follows from (4.6) and the inequality follows from Lemma 4.1.

We have stated that when the division $M$ of $N$, derived from $\left(N, \sigma_{0}, w, p\right)$ and $\rho$, is formed of singletons, it holds that

$$
v(S ; \rho)=\sum_{i \in S} E G S_{i}\left(N, \sigma_{0}, w, p\right)
$$

for any $S \in \rho$. Next, we show a more general result by our next corollary, which can be immediately obtained from the proof of Theorem 4.5 and Lemma 4.2. 
Corollary 4.2. Let $\left(N, \sigma_{0}, w, p\right)$ be a sequencing situation and $(N, v)$ the corresponding partition sequencing game. Given a coalition $S \subseteq N$ and a partition $\rho$ of $N$ with $S \in \rho$,

$$
v(S ; \rho)=\sum_{i \in S} E G S_{i}\left(N, \sigma_{0}, w, p\right),
$$

if and only if there exists an optimal order $\hat{\sigma}$ such that all groups in the division derived from $\left(N, \sigma_{0}, w, p\right)$ and $\rho$ are simple with respect to $\hat{\sigma}$.

The next theorem shows that the allocation yielded by the EGS rule is the only core element if the core of the partition sequencing game is based on the s-exogenous rule.

Theorem 4.6. Let $\left(N, \sigma_{0}, w, p\right)$ be a sequencing situation and $(N, v)$ the corresponding partition sequencing game. Then

$$
C_{R^{s}}(v)=\left\{E G S\left(N, \sigma_{0}, w, p\right)\right\} .
$$

Proof. From Theorem 4.5 we have $\operatorname{EGS}\left(N, \sigma_{0}, w, p\right) \in C_{R^{s}}(v)$. To complete the proof, it is sufficient to show that for any solution $\varphi \in C_{R^{s}}(v), \varphi=$ $E G S\left(N, \sigma_{0}, w, p\right)$. Let $\varphi \in C_{R^{s}}(v)$. Observe that for any $i \in N$

$$
\varphi_{i} \geq v\left(\{i\} ; R^{S}(\{i\})\right)=v(\{i\} ;[N])
$$

Then we must have

$$
v(N ;\{N\})=\sum_{i \in N} \varphi_{i} \geq \sum_{\{i\} \in[N]} v(\{i\} ;[N])=v(N ;\{N\}),
$$

where the last equality follows from Corollary 4.1. Hence, for any $i \in N$, we have $\varphi_{i}=v(\{i\} ;[N])$.

Let $M$ be the division derived, and $\pi$ the group order derived from $\left(N, \sigma_{0}, w, p\right)$ 
and partition $[N]$. Using the definition of partition sequencing games it follows that

$$
\begin{aligned}
v(\{i\} ;[N]) & =\frac{1}{2}\left(\sum_{\{j\} \in P(\pi,\{i\})} G_{\{j\}\{i\}}+\sum_{\{k\} \in F(\pi,\{i\})} G_{\{i\}\{k\}}\right) \\
& =E G S_{i}\left(N, \sigma_{0}, w, p\right) .
\end{aligned}
$$

Therefore, $\varphi=E G S\left(N, \sigma_{0}, w, p\right)$.

\subsection{A noncooperative implementation of the EGS rule}

The EGS rule proposed by Curiel et al. [21] has many nice properties for solving the cost savings allocation problem in sequencing situations. Even in the environment where the externalities exist, it is easy to see from the previous section that the EGS rule provides a more fair and stable payoff distribution, since it always yields a core element no matter which partition rule is applied to the corresponding partition sequencing game.

In this section, we introduce and analyze a mechanism that implements the EGS rule in partition sequencing games. We now formally describe the mechanism, which is defined recursively with the following extensive form.

\section{The mechanism:}

Let $\left(N, \sigma_{0}, w, p\right)$ be a sequencing situation, and $(N, v)$ the corresponding partition sequencing game. A protocol $\sigma \in \Omega_{N}$ is an order of players that prescribes the order of moves in the mechanism. In each period $t$, let $N^{t}$ be the set of active players, where $N^{1}=N$. Denote by $i^{t}$ the player who is the proposer in period $t$. We define $i^{1}=\sigma^{-1}(1)$, that is, the first proposer is the player in the first position of $\sigma$. Then the game in each period consists of the following three stages.

Stage $1 i^{t}$ proposes a coalition $K \subseteq N^{t}$ with $i^{t} \in K$ and an offer $y_{j} \in \mathbb{R}$ for each player $j \in K \backslash\left\{i^{t}\right\}$. Go to stage 2 .

Stage 2 All the players in $K$ other than the proposer respond sequentially 
by either accepting or rejecting. We prescribe that for any respondents $i, j \in K \backslash\left\{i^{t}\right\}$ with $\sigma(i)<\sigma(j), i$ will respond before $j .{ }^{5}$ If all the other players accept the proposal, coalition $K$ forms and each player $j \in K \backslash\left\{i^{t}\right\}$ receives $y_{j}$. Then the proposer $i^{t}$ obtains $v(K ; \rho)-$ $\sum_{j \in K \backslash i^{t}} y_{j}$, where $\rho$ is the coalition structure formed by all players after the whole process stops. This means that the proposer cannot really receive any payoff until the game ends. Then $N^{t+1}=N^{t} \backslash K$ becomes the new active player set, and the player in the most forward position among all the active players with respect to $\sigma$ becomes the new proposer, i.e., $i^{t+1}=\arg \min _{i \in N^{t+1}} \sigma(i)$. Then the game goes to stage 1 of the next period. If there is one player, say $k$, being the first one to reject the proposal, the game goes to the next stage.

Stage 3 The proposer $i^{t}$ leaves the game and forms a coalition containing only himself. Then the current active player set is $N^{t+1}=N^{t} \backslash\left\{i^{t}\right\}$. Player $i^{t}$ has to wait for the game to complete so he can obtain his final payoff $v(\{i\} ; \rho)$, where $\rho$, again, is the coalition structure formed by all players after the whole process stops. The first rejecter $k$ now becomes a new proposer, i.e., $k=i^{t+1}$, and the game goes to stage 1 of the next period.

The whole process described above stops when the active player set is empty. Observe that there is always a coalition containing at least one player to be formed in each period, a coalition structure $\rho$ (a partition) will eventually form after the game ends. Beyond that, it is easy to see that the game will cover at most $|N|$ periods (at least one period) and therefore our mechanism admits a finite extensive form game. For any active player set $S$, denote by $\rho_{N \backslash S}$ the coalition structure that has already been formed by the players in $N \backslash S$. The subgame with the protocol $\sigma$, the active player set $S$ and the partition $\rho_{N \backslash S}$ is denoted by $\Gamma^{\sigma}\left(S, \rho_{N \backslash S}\right)$. A (pure) strategy for player $i$ in $\Gamma^{\sigma}\left(S, \rho_{N \backslash S}\right)$ is a sequence $\eta_{i}=\left(\eta_{i}^{t}\right)_{t=1}^{|N|}$ of mappings, where $\eta_{i}^{t}$ prescribes a proposal and a response function assigning "accept" or "reject" to all possible

\footnotetext{
${ }^{5}$ In fact, the sequential ordering of the respondents is nonsignificant. Changing the sequencing of the respondents will not affect the final outcome.
} 
proposals by other players. A strategy profile $\eta^{S}=\left(\eta_{i}\right)_{i \in S}$ is called a subgame perfect equilibrium (SPE) in $\Gamma^{\sigma}\left(S, \rho_{N \backslash S}\right)$ if for any player $i \in S$ and any history of play, $\eta_{i}$ is a best-response for $i$.

One may notice that in our mechanism, it seems that a proposer cannot be sure how much payoff he will obtain, since his final payoff is also determined by the strategies of the remaining players. And it also seems that a respondent will have trouble judging whether to accept or reject a proposal, since the objection will certainly make him a new proposer and face an uncertain payoff. However, we will show by our next theorem that for any protocol $\sigma$ and any subgame $\Gamma^{\sigma}\left(S, \rho_{N \backslash S}\right)$ with $S \subseteq N \backslash\left\{i^{1}\right\}$, if there exists an optimal order $\hat{\sigma}$ such that all groups in the division of $N \backslash S$, derived from coalition structure $\rho_{N \backslash S}$ and the sequencing situation $\left(N, \sigma_{0}, w, p\right)$, are simple with respect to $\hat{\sigma}$, then the unique SPE payoff profile for the players in $S$ corresponds to the allocation yielded by the EGS rule.

Theorem 4.7. Let $\left(N, \sigma_{0}, w, p\right)$ be a sequencing situation. For any protocol $\sigma$ and any subgame $\Gamma^{\sigma}\left(S, \rho_{N \backslash S}\right)$ with $S \subseteq N \backslash\left\{i^{1}\right\}$, if there exists an optimal order $\hat{\sigma}$ such that all groups in the division of $N \backslash S$, derived from $\rho_{N \backslash S}$ and $\left(N, \sigma_{0}, w, p\right)$, are simple with respect to $\hat{\sigma}$, then there exists an $\operatorname{SPE}$ in $\Gamma^{\sigma}\left(S, \rho_{N \backslash S}\right)$, and for each $S P E$ in $\Gamma^{\sigma}\left(S, \rho_{N \backslash S}\right)$ the equilibrium payoff for any $i \in S$ is $E G S_{i}\left(N, \sigma_{0}, w, p\right)$.

Proof. The proof proceeds by induction on the number of players in the active player set $S$. For $S=\{i\}$, the theorem obviously holds since from Corollary 4.2 we know that $v\left(\{i\} ;\{i\} \cup \rho_{N \backslash\{i\}}\right)=E G S_{i}\left(N, \sigma_{0}, w, p\right)$.

We now assume that the theorem holds for $|S|=s-1$ and show that it also holds for $|S|=s$. Suppose all groups in the division of $N \backslash S$, derived from $\rho_{N \backslash S}$ and $\left(N, \sigma_{0}, w, p\right)$, are simple with respect to an optimal order $\hat{\sigma}$. We now construct a strategy profile as follows:

(i) At stage 1, if $i \in S$ is the proposer, he proposes a coalition $K \subseteq S$ satisfying that $i \in K$ and the $\sigma_{0}$-components of $K$ are simple groups with respect to $\hat{\sigma}$. Then he offers $y_{j}=E G S_{j}\left(N, \sigma_{0}, w, p\right)$ to every $j \in K \backslash\{i\}$.

(ii) At stage 2, if $j$ is a respondent, he accepts the ongoing proposal if and only if the offer satisfies $y_{j} \geq E G S_{j}\left(N, \sigma_{0}, w, p\right)$. 
We now show by backward induction that previous strategy profile constitute an SPE . At stage 2, any respondent $j$ will certainly accept any offer that is not less than $E G S_{j}\left(N, \sigma_{0}, w, p\right)$. This is because if he rejects the proposal, the proposer $i$ will leave the game and form a singleton by himself. Note that all groups in the division of $N \backslash S \cup\{i\}$, derived from $\rho_{N \backslash S} \cup\{\{i\}\}$ and $\left(N, \sigma_{0}, w, p\right)$, are simple with respect to $\hat{\sigma}$, by the induction argument, the unique equilibrium payoff for $j$ in the subgame $\Gamma^{\sigma}\left(S \backslash\{i\}, \rho_{N \backslash S} \cup\{\{i\}\}\right)$ is $E G S_{j}\left(N, \sigma_{0}, w, p\right)$. This implies that the action in (ii) cannot be improved upon.

We then show the action in (i) is not improvable. Consider the respondents' actions in (ii), the proposal will be unanimously accepted and thus $K$ will form. Let $\rho_{S \backslash K}$ be the partition of $S \backslash K$ formed by following some SPE in $\Gamma^{\sigma}\left(S \backslash K, \rho_{N \backslash S} \cup\{K\}\right)$. Since $|K| \geq 1$ and the $\sigma_{0}$-components of $K$ are simple groups with respect to $\hat{\sigma}$, by the induction argument, any player $i$ in the subgame $\Gamma^{\sigma}\left(S \backslash K, \rho_{N \backslash S} \cup\{K\}\right)$ will obtain his equilibrium payoff $E G S_{i}\left(N, \sigma_{0}, w, p\right)$. Then we can derive that in the formation of any coalition $T \in \rho_{S \backslash K}$, the following equation must hold,

$$
v\left(T ; \rho_{N \backslash S} \cup\{K\} \cup \rho_{S \backslash K}\right)=\sum_{i \in T} E G S_{i}\left(N, \sigma_{0}, w, p\right) .
$$

By Corollary 4.2, we can conclude that (4.10) implies that there exists an optimal order $\hat{\sigma}^{\prime}$ such that all groups in the division of $N$, derived from $\rho_{N \backslash S} \cup\{K\} \cup \rho_{S \backslash K}$ and $\left(N, \sigma_{0}, w, p\right)$ are simple with respect to $\hat{\sigma}^{\prime}{ }^{6}$ Using Corollary 4.2 again, we have

$$
v\left(K ; \rho_{N \backslash S} \cup\{K\} \cup \rho_{S \backslash K}\right)=\sum_{i \in K} E G S_{i}\left(N, \sigma_{0}, w, p\right) .
$$

Hence, the final payoff for the proposer $i$ is

$$
v\left(K ; \rho_{N \backslash S} \cup\{K\} \cup \rho_{S \backslash K}\right)-\sum_{j \in K \backslash\{i\}} E G S_{j}\left(N, \sigma_{0}, w, p\right)=E G S_{i}\left(N, \sigma_{0}, w, p\right) .
$$

Let us now consider a deviation from the action in (i):

\footnotetext{
${ }^{6}$ Whether or not $\hat{\sigma}^{\prime}$ coincides with $\hat{\sigma}$ does not have any effect on the following result.
} 
Case 1: Suppose that the coalition proposed by the proposer $i$ does not change but the offer for at least one respondent, say $k$, is not $E G S_{k}\left(N, \sigma_{0}, w, p\right)$.

Subcase 1: $y_{j} \geq E G S_{j}\left(N, \sigma_{0}, w, p\right)$ for any $j \in K \backslash\{i\}$ and for $k \in K \backslash\{i\}$, $y_{k}>E G S_{k}\left(N, \sigma_{0}, w, p\right)$. According to the action in (ii), the proposal will be accepted by all the respondents and $K$ will form. From (4.11) we know that the worth of the coalition $K$ is $\sum_{j \in K} E G S_{j}\left(N, \sigma_{0}, w, p\right)$, which implies that the final payoff for $i$ is strictly less than $E G S_{i}\left(N, \sigma_{0}, w, p\right)$.

Subcase 2: $y_{k}<E G S_{k}\left(N, \sigma_{0}, w, p\right)$. According to the action in (ii), $k$ will certainly reject the proposal and make $i$ a singleton. By simply changing " $K$ " to " $\{i\}$ ", we can use the same analysis like we did for concluding (4.11) to show that the final payoff for $i$ is still $E G S_{i}\left(N, \sigma_{0}, w, p\right)$.

Case 2: Suppose that the coalition proposed by $i$ is $T \subseteq S$ with $T \neq K$. From Theorem 4.5 we can see that if the coalition $T$ forms, the worth of $T$ will be no greater than $\sum_{j \in T} E G S_{j}\left(N, \sigma_{0}, w, p\right)$ no matter how the rest of game is performed by the remaining players. If the proposer chooses to make $T$ formed, then he has to offer every $j \in T \backslash\{i\}$ at least $E G S_{j}\left(N, \sigma_{0}, w, p\right)$ for the proposal to be accepted. However, in this case the final payoff for $i$ will be no more than $E G S_{i}\left(N, \sigma_{0}, w, p\right)$. If the offer to some player $k \in T \backslash\{i\}$ is less than $E G S_{k}\left(N, \sigma_{0}, w, p\right)$, by the same argument as in the Subcase 2 of Case 1 , the final payoff for $i$ is still $E G S_{i}\left(N, \sigma_{0}, w, p\right)$.

We now show that for each SPE in $\Gamma^{\sigma}\left(S, \rho_{N \backslash S}\right)$, the equilibrium payoff for any $i \in S$ is $E G S_{i}\left(N, \sigma_{0}, w, p\right)$. Given any SPE $\eta$ in $\Gamma^{\sigma}\left(S, \rho_{N \backslash S}\right)$, let $u^{S}$ be the corresponding equilibrium payoff vector for $S, i^{*}$ the first proposer in $\Gamma^{\sigma}\left(S, \rho_{N \backslash S}\right), T^{*}$ the coalition formed from the proposal of $i^{*}$ by following the SPE $\eta$, and $\rho_{S \backslash T^{*}}$ the partition of $S \backslash T^{*}$ resulted from the SPE $\eta$.

Claim 1. For any $i \in T^{*}, u_{i}^{S} \geq E G S_{i}\left(N, \sigma_{0}, w, p\right)$.

This result is straightforward for $i^{*}$ since if $u_{i^{*}}^{S}<E G S_{i^{*}}\left(N, \sigma_{0}, w, p\right), i^{*}$ can simply change his strategy in $\eta$ by proposing a coalition $\left\{i^{*}\right\}$. In this way, by the induction argument and (4.11), the final payoff for $i^{*}$ is $E G S_{i^{*}}\left(N, \sigma_{0}, w, p\right)$. For any $j \in T^{*} \backslash\left\{i^{*}\right\}$, if $u_{j}^{S}<E G S_{j}\left(N, \sigma_{0}, w, p\right)$, $j$ can reject the proposal and make $i^{*}$ a singleton. Then he becomes a new proposer and proposes the coalition $\{j\}$. Similarly, in this way, the final payoff for $j$ is $E G S_{j}\left(N, \sigma_{0}, w, p\right)$. 
Claim 2. There exists an optimal order $\hat{\sigma}^{\prime}$ such that all groups in the division of $N$ derived from $\rho_{N \backslash S} \cup\left\{T^{*}\right\} \cup \rho_{S \backslash T^{*}}$ and $\left(N, \sigma_{0}, w, p\right)$ are simple with respect to $\hat{\sigma}^{\prime}$.

Suppose not. According to Corollary 4.2, we know that the final worth of $T^{*}$ must be strictly less than $\sum_{i \in T^{*}} E G S_{i}\left(N, \sigma_{0}, w, p\right)$. However, from Claim 1 it holds that

$$
v\left(T^{*} ; \rho_{N \backslash S} \cup\left\{T^{*}\right\} \cup \rho_{S \backslash T^{*}}\right)=\sum_{i \in T^{*}} u_{i}^{S} \geq \sum_{i \in T^{*}} E G S_{i}\left(N, \sigma_{0}, w, p\right) .
$$

A contradiction.

By the induction argument and Claim 2, we can immediately conclude that for any $j \in S \backslash T^{*}, u_{j}^{S}=E G S_{j}\left(N, \sigma_{0}, w, p\right)$. Moreover, from Claim 2 and Corollary 4.2 we can also have

$$
v\left(T^{*} ; \rho_{N \backslash S} \cup\left\{T^{*}\right\} \cup \rho_{S \backslash T^{*}}\right)=\sum_{i \in T^{*}} E G S_{i}\left(N, \sigma_{0}, w, p\right) .
$$

Combining the above equality with Claim 1 we have for any $i \in T^{*}, u_{i}^{S}=$ $E G S_{i}\left(N, \sigma_{0}, w, p\right)$.

Theorem 4.7 provides a nice approach for the first proposer $i^{1}$ to have a sharp prediction on the behavior of remaining players. It is easy to see that the optimal strategy of $i^{1}$ for reaching his maximal payoff is to propose a coalition having simple groups and offer each player $j \neq i^{1}$ in that coalition $E G S_{j}\left(N, \sigma_{0}, w, p\right) .^{7}$ Consequently, by using Theorem 4.7 again, we know that the unique equilibrium payoff for any player $i \in N$ is $E G S_{i}\left(N, \sigma_{0}, w, p\right)$. This result can be shown by the following theorem, the proof of which is in the same way as we prove Theorem 4.7 and thus can be omitted here.

Theorem 4.8. Let $\left(N, \sigma_{0}, w, p\right)$ be a sequencing situation. For any protocol $\sigma$, the mechanism $\Gamma^{\sigma}(N, \emptyset)$ implements the EGS rule in SPE.

\footnotetext{
${ }^{7}$ Another optimal strategy for $i^{1}$ is to propose a proposal that will always be rejected for making himself a singleton.
} 



\section{Chapter 5}

\section{A noncooperative bargaining game with endogenous protocol and partial breakdown}

In this chapter, we present a noncooperative bargaining game with transferable utility. Instead of assuming that the recognition of proposers is determined randomly or by a fixed exogenous protocol, we provide a mechanism in which the protocol is generated endogenously. We consider a partial breakdown probability rather than a discount factor when the proposal is rejected by some player. We show that for each partial breakdown probability, stationary subgame perfect equilibria exist if the characteristic function game is totally balanced. Moreover, the outcomes of stationary subgame perfect equilibria in our model coincide with the core allocations. 


\subsection{Introduction}

This chapter aims to explore a noncooperative method to model $n$-person coalitional bargaining game with transferable utility and discover the approach to no-delay and efficient agreements as well as fair payoff allocations.

Since the publication of the seminal paper by Rubinstein [75], coalitional bargaining has been a very active research direction in game theory. Chatterjee et al. [15] extend the two-person alternating-offer model of [75] to the multi-person situation, where an exogenous protocol, which is a fixed order over players, plays an essential role in determining the outcomes. Hereafter, from different perspectives, the spirit of the protocol is widely used by several studies including [7], [53], [73], but also invites arguments such as being too specific on the selection of the first proposer. Focusing on this shortage, Okada [68] proposes a stationary bargaining game in which the proposer is randomly recognized with equal probability from all players, and this randomization mechanism is further generalized in [70]. Other contributions that involve the design of random-proposer can be found in [32], [45], [69], [91]. Despite a good attempt for avoiding being partial to some players in the bargaining, the randomization mechanism has the same defect as the fixed order protocol: the recognition probabilities of proposers are exogenous, which means that they are artificially fixed before the game getting started. Then, this arises a natural question which has already been mentioned in [68]: "how is the protocol actually determined?" Or in other words, is there some mechanism that determines proposers endogenously?

With such a question in mind, this chapter puts forward a method for the selections of proposers being endogenously determined by the strategies of players. In our model, at the first stage of each period, every active player simultaneously proposes a feasible payoff vector and a permutation of players, if all active players choose the same payoff vector, the game goes to the next stage and the combination of permutations of active players becomes the current protocol. At the second stage, the bargaining process continues the same procedure as in [15]: the first player in the active player set under the current protocol becomes the proposer. Then he makes a proposal containing a coalition (which contains the proposer himself) and a feasible 
payoff vector for the coalition. All other players in the coalition respond sequentially according to the current protocol. If they all accept the proposal, then they leave the game with the allocation which has been a consensus within the coalition. The remaining players become active and continue the negotiations in the next period.

Another main distinguishing feature of our game model appears in the situation when the proposal is rejected by some member of the coalition. Instead of appointing the first rejector as the new proposer with certainty, we introduce the concept of partial breakdown probability (PBP) for distinguishing two events that may happen. The first rejector may become the new proposer with a certain probability. Whereas in another case, the coalition could also be swept out of the game with the allocation determined in the first stage and the remaining players continue the negotiations at the next period. It's worth mentioning that our model has no restriction about discounting, which is a general assumption in the literature and prescribes that players discount their future payoffs by a common discount factor. Instead of imposing this strong assumption, which compulsively reduces the payoff of players if there are delays in the negotiation process, in our model, we let the rejector face the risk of getting the payoff determined in the first stage. Our main result shows that for each PBP, stationary subgame perfect equilibrium payoff set coincides with the core if the characteristic function game is totally balanced.

We briefly mention that this chapter is not the first one to attempt to endogenize the bargaining protocol. For example, the mechanism proposed by Pérez-Castrillo and Wettstein [72] for implementing the Shapley value includes a round of biddings to select the proposer. Evans [28] and Yildirim [93] allowed players to make investments at a cost to become the proposer. Serrano and Vohra [77] and Chang and $\mathrm{Hu}$ [14] use the same proposer-selecting process as we do in the first stage of our model. Among those literature, [77] perhaps is the most relevant to our study, since they also analyze the mechanism, which involves the endogenous protocol and implements the core. However, we will compare our model and results with theirs in Section 5.4 and show that in some sense, their bargaining game can be regarded as a special case of ours when we restrict the PBP to an extreme condition.

The chapter is organized as follows. In Section 5.2, we introduce our 
noncooperative bargaining model. Section 5.3 presents our main results. In Section 5.4, some further discussions are involved.

\subsection{The model}

Our noncooperative bargaining model is a dynamic game, denoted by $G^{\epsilon}(N)$, where $\epsilon=\left(\epsilon_{i}\right)_{i \in N}$ is a vector and for each $i \in N, \epsilon_{i} \in(0,1)$. In our game, the parameter $\epsilon_{i}$ is only related to the player $i$ himself and is referred to as the partial breakdown probability ${ }^{1}$ (PBP) of player $i$. The model has the following process:

In each period $t=1,2, \cdots$, let $N^{t}$ be the set of active players, where $N^{1}=$ $N$. The bargaining game in each period consists of two stages.

Stage 1 Every player in $N^{t}$ chooses simultaneously from the choice set $X\left(N^{t}\right) \times$ $\Theta\left(N^{t}\right)$. That is, each player can choose generic elements from the feasible payoff set and the permutation set. A typical choice of a player $i$ is denoted by $\left(x^{N^{t} \mid i}, \theta^{N^{t} \mid i}\right)$, which refers to player $i$ 's proposition of an allocation and a play order for the current active players. Let $\theta^{N^{t}}$ be the composition of $\left(\theta^{N^{t} \mid i}\right)_{i \in N^{t}}$. Then one of the following two cases can happen:

Case 1 If $x^{N^{t} \mid k} \neq x^{N^{t} \mid l}$ for some $k, l \in N^{t}$, player $\theta^{N^{t}}(1)$ obtains the outcome $d<0$ and all the other players receive 0 . Then the whole game process stops after this stage.

Case 2 If $x^{N^{t} \mid k}=x^{N^{t} \mid l}=x^{N^{t}}$ for any $k, l \in N^{t}$, the game proceeds to the next stage. $x^{N^{t}}$ here is referred to as the status-quo.

Stage 2 Suppose that $\theta^{N^{t}}(1)=i$, $i$ becomes the proposer. Then he must propose a proposal $\left(S, y^{S}\right)$, where $S \subseteq N^{t}$ is a nonempty coalition containing himself and $y^{S} \in X(S)$ is a feasible payoff for $S$. All the

\footnotetext{
${ }^{1}$ The breakdown probability is also involved to implement the Nash bargaining solution in the bargaining model provided by [69], where the whole negotiation process will stop if the breakdown happens. Other studies considering partial breakdowns include [42], [57], [50]. In contrast to our study, these literature allow only one player to exit from the game when the partial breakdown happens.
} 
other players in $S$ respond sequentially according to the permutation $\theta^{N^{t}}$ (starting with player $\theta^{N^{t}}(2)$ and going up to $\theta^{N^{t}}(s)$ ) by either accepting or rejecting. Then there are also two cases:

Case 1 If all the other players accept the proposal, then coalition $S$ leaves the game with the allocation $y^{S} . N^{t} \backslash S$ becomes the set of active players and continues the game at the next period.

Case 2 If there is a player, say $j \in S$, being the first one to reject the proposal, with probability $\epsilon_{j} \in(0,1)$ all the players in $S$ leave the game and they receive the payoff vector which is a projection of $x^{N^{t}}$ to the subspace corresponding to $S$. Then $N^{t} \backslash S$ becomes the set of active players and continues the game in the next period. With probability $1-\epsilon_{j}, j$ may become a new proposer and choose a new proposal.

At Stage 1, our mechanism requires that all the active players should coordinate their actions of choosing an identical feasible payoff. Otherwise, the game ends, and all the active players receive undesirable payoffs, especially the player in the first position of the endogenous protocol. It should be noted that the selection of active proposers at Stage 2 of each period is entirely dependent on the choices of the active players in the previous stage. At Stage 2, the PBP of a player can be seen as his personal prediction for the possibility of the departure of the coalition being proposed if he rejects. It is assumed that the PBPs of all players are common information. We denote by $G^{\epsilon}(S)$ the subgame of $G^{\epsilon}(N)$ with the active player set $S$ and the PBP vector $\epsilon$.

In this and also the following chapter, we restrict our attention to stationary subgame perfect equilibria (SSPE). A (pure) strategy $\eta_{i}$ for any player $i$ is stationary if for each period $t$, the decisions of $i$ depend only on the set $N^{t}$, the status-quo $x^{N^{t}}$ and the ongoing proposal when $i$ is a respondent. In other words, a stationary strategy does not condition on the history of past periods. Given any stationary strategy and active player set $S$, we denote by $u_{i}^{S}$ the expected equilibrium payoff to $i$ if $i$ is the proposer when all players in $S$ implement $\eta . u^{S}=\left(u_{i}^{S}\right)_{i \in S}$ will be called the proposer payoff vector with 
respect to strategy profile $\eta$. A stationary strategy profile $\eta=\left(\eta_{i}\right)_{i \in N}$ is nodelay if after any history, the proposer makes a proposal that will certainly be accepted according to $\eta$. A stationary strategy profile $\eta=\left(\eta_{i}\right)_{i \in N}$ is called a stationary subgame perfect equilibrium if for each player $i$ and each history of play, $\eta_{i}$ is a best-response for $i$.

The reason that we are looking for SSPE is that the set of non-stationary subgame perfect equilibrium outcomes in a broad class of Rubinstein-type sequential infinite bargaining games are infinitely large (see [71]). The results of Herrero [43] and Chatterjee et al. [15] suggest that, in the context of nperson infinite bargaining games of that type, any feasible allocation can be supported by non-stationary subgame perfect equilibria. Therefore, it is not of much help to sharpen feasible equilibria if it is not coupled with stationarity. However, it has been recognized that stationarity can typically select a unique equilibrium (see, for example, [3], [75]). Moreover, the stationary equilibrium has good properties such as being stable for nonstationary deviations (see [76]). Thus, we restrict our attention, as many other authors do, to SSPE.

Since the case $n=1$ is trivial, the bargaining game we consider in the current chapter with the number of players $n \geq 2$.

\subsection{Main results}

Let $(N, v)$ be a characteristic function game. Our first theorem discusses the existence of a no-delay SSPE which implements a core allocation.

Theorem 5.1. Let $(N, v)$ be a characteristic function game and $G^{\epsilon}(N)$ the corresponding bargaining game. If $(N, v)$ is totally balanced, then for each $\epsilon \in(0,1)^{n}$ and $y^{N} \in C(v)$, there exists a no-delay SSPE implementing $y^{N}$.

Before we prove Theorem 5.1, it will be helpful to define a few terms. Fix a player set $S$ and a feasible payoff vector $x^{S} \in X(S)$. Let $M^{S}=\left[0, \max _{T \subseteq S} v(T)\right]^{s}$ be the $s$-dimensional subspace of $\mathbb{R}_{+}^{s}$, that is, the cube in $\mathbb{R}_{+}^{s}$ with vertex 0 and 
length $\max _{T \subseteq S} v(T)$. Denote $\phi$ for the function from $M^{s}$ to $M^{s}$ defined by

$$
\phi_{i}(m)=\max _{i \in T \subseteq S}\left\{v(T)-\sum_{j \in T \backslash i}\left[\epsilon_{j} x_{j}^{S}+\left(1-\epsilon_{j}\right) m_{j}\right]\right\},
$$

where $m \in M^{S}$ is a vector, i.e., $m=\left\{m_{j}\right\}_{j \in S}$. Here, we simply explain the intuition behind expression (5.1). Consider $m_{j}$ to be the maximal payoff player $j$ can get if he becomes the proposer. Then $\epsilon_{j} x_{j}^{S}+\left(1-\epsilon_{j}\right) m_{j}$ is the expected payoff when $j$ refuses to take the proposal from $i$. Any offer less than $\epsilon_{j} x_{j}^{S}+\left(1-\epsilon_{j}\right) m_{j}$ when $j$ is a respondent shall be rejected. We can see that the expression (5.1) gives the solution for each proposer $i$ to achieve his maximal payoff when he wants his proposal to be accepted. The key point of proving Theorem 5.1 is to find a fixed point of $\phi . M^{S}$ guarantees that values of the recursive construction are still in it. The fixed point argument for the existence of a no-delay SSPE can also be found in [15] and [73].

The following lemmas are also needed.

Lemma 5.2. For each active player set $S \subseteq N$ and each $x^{S} \in X(S)$, $\phi$ has a fixed point, i.e., $\phi(m)=m$.

Proof. For each player $i$ and $T \subseteq S$ with $i \in T$, define the function $f_{i}^{T}(m)$ as follows:

$$
f_{i}^{T}(m)=v(T)-\sum_{j \in T \backslash i}\left[\epsilon_{j} x_{j}^{S}+\left(1-\epsilon_{j}\right) m_{j}\right] .
$$

Clearly, $f^{T}(m)$ is a continuous function. Since the maximum of continuous functions is a continuous function, we have that function $\phi$ is continuous. Because $M^{S}$ is a compact and convex set, and $\phi$ is continuous, it follows from Brouwer's fixed point Theorem [11] that $\phi$ has at least one fixed point.

Lemma 5.3. For each active player set $S \subseteq N$ with $i \in S$ and $x^{S} \in X(S)$, let $\hat{m}$ be a fixed point of $\phi$ and $T_{i} \subseteq S$ be the coalition containing $i$ and attaining the maximum in (5.1). Then for each $j \in T_{i}$, we have $\epsilon_{i}\left(\hat{m}_{i}-x_{i}^{S}\right) \leq \epsilon_{j}\left(\hat{m}_{j}-x_{j}^{S}\right)$. 
Proof. From Lemma 5.2, we can guarantee the existence of $\hat{m}$. Then for player $i$, we must have

$$
\hat{m}_{i}=v\left(T_{i}\right)-\sum_{j \in T_{i} \backslash i}\left[\epsilon_{j} x_{j}^{S}+\left(1-\epsilon_{j}\right) \hat{m}_{j}\right]
$$

and for player $j \in T_{i} \backslash i$ we have

$$
\hat{m}_{j} \geq v\left(T_{i}\right)-\sum_{k \in T_{i} \backslash j}\left[\epsilon_{k} x_{k}^{S}+\left(1-\epsilon_{k}\right) \hat{m}_{k}\right] .
$$

By adding $-\left[\epsilon_{j} x_{j}^{S}+\left(1-\epsilon_{j}\right) \hat{m}_{j}\right]$ on both sides of (5.3) and using (5.2) we have

$$
\begin{aligned}
\epsilon_{j}\left(\hat{m}_{j}-x_{j}^{S}\right) & \geq v\left(T_{i}\right)-\sum_{k \in T_{i} \backslash j}\left[\epsilon_{k} x_{k}^{S}+\left(1-\epsilon_{k}\right) \hat{m}_{k}\right]-\left[\epsilon_{j} x_{j}^{S}+\left(1-\epsilon_{j}\right) \hat{m}_{j}\right] \\
& =v\left(T_{i}\right)-\sum_{k \in T_{i} \backslash i}\left[\epsilon_{k} x_{k}^{S}+\left(1-\epsilon_{k}\right) \hat{m}_{k}\right]-\left[\epsilon_{i} x_{i}^{S}+\left(1-\epsilon_{i}\right) \hat{m}_{i}\right] \\
& =\hat{m}_{i}-\left[\epsilon_{i} x_{i}^{S}+\left(1-\epsilon_{i}\right) \hat{m}_{i}\right] \\
& =\epsilon_{i}\left(\hat{m}_{i}-x_{i}^{S}\right) .
\end{aligned}
$$

Lemma 5.4. For each active player set $S \subseteq N$ and $x^{S} \in X(S)$, let $\hat{m}$ be a fixed point of $\phi$. If $x^{S} \in C\left(v^{S}\right)$, then $\hat{m}=x^{S}$.

Proof. (If). We first show that $\hat{m}_{i} \leq x_{i}^{S}$ for each $i \in S$. Given any player $i \in S$, let $T_{i}$ be the coalition such that

$$
\hat{m}_{i}=v\left(T_{i}\right)-\sum_{j \in T_{i} \backslash i}\left[\epsilon_{j} x_{j}^{S}+\left(1-\epsilon_{j}\right) \hat{m}_{j}\right] .
$$

Since $x^{S} \in C\left(v^{S}\right)$, we must have

$$
\hat{m}_{i} \leq \sum_{j \in T_{i}} x_{j}^{S}-\sum_{j \in T_{i} \backslash i}\left[\epsilon_{j} x_{j}^{S}+\left(1-\epsilon_{j}\right) \hat{m}_{j}\right] .
$$


By adding $-x_{i}^{S}$ on both sides of (5.4) we have

$$
\begin{aligned}
\hat{m}_{i}-x_{i}^{S} & \leq \sum_{j \in T_{i} \backslash i} x_{j}^{S}-\sum_{j \in T_{i} \backslash i}\left[\epsilon_{j} x_{j}^{S}+\left(1-\epsilon_{j}\right) \hat{m}_{j}\right] . \\
& =\sum_{j \in T_{i} \backslash i}\left(\epsilon_{j}-1\right)\left(\hat{m}_{j}-x_{j}^{S}\right) .
\end{aligned}
$$

Now suppose that $\hat{m}_{i}>x_{i}^{S}$. Since $\epsilon_{i} \in(0,1)$ for each $i \in N$, it follows from Lemma 5.3 that $\hat{m}_{j}>x_{j}^{S}$ for each $j \in T_{i}$. Hence the inequality (5.5) can not hold, a contradiction.

Then we show that $\sum_{i \in S} \hat{m}_{i} \geq v(S)$. Since $\hat{m}$ is a fixed point of function $\phi$, for every player $i \in S$ there must exist a coalition $T_{i}$ which contains $i$ such that

$$
\begin{aligned}
\hat{m}_{i} & =\max _{i \in T \subseteq S}\left\{v(T)-\sum_{j \in T \backslash i}\left[\epsilon_{j} x_{j}^{S}+\left(1-\epsilon_{j}\right) \hat{m}_{j}\right]\right\} \\
& =v\left(T_{i}\right)-\sum_{j \in T_{i} \backslash i}\left[\epsilon_{j} x_{j}^{S}+\left(1-\epsilon_{j}\right) \hat{m}_{j}\right] .
\end{aligned}
$$

Then the following inequality obviously holds,

$$
\hat{m}_{i} \geq v(S)-\sum_{j \in S \backslash i}\left[\epsilon_{j} x_{j}^{S}+\left(1-\epsilon_{j}\right) \hat{m}_{j}\right] .
$$

Sum up (5.6) for all players in $S$, we get

$$
\begin{aligned}
\sum_{j \in S} \hat{m}_{j} & \geq s v(S)-(s-1) \sum_{j \in S}\left[\epsilon_{j} x_{j}^{S}+\left(1-\epsilon_{j}\right) \hat{m}_{j}\right] \\
& \geq s v(S)-(s-1) \sum_{j \in S} x_{j}^{S} \\
& \geq s v(S)-(s-1) v(S) \\
& =v(S),
\end{aligned}
$$

where the second inequality follows from the fact that $\hat{m}_{j} \leq x_{j}^{S}$ for each $j \in S$, and the third inequality follows from $x^{S} \in X(S)$. Since $x^{S} \in C\left(v^{S}\right)$, we have

$$
v(S)=\sum_{j \in S} x_{j}^{S} \geq \sum_{j \in S} \hat{m}_{j} \geq v(S) .
$$


Hence $\hat{m}_{j}=x_{j}^{S}$ for each $j \in S$.

Proof of Theorem 5.1. We first construct a no-delay strategy profile $\eta$ as follows:

Suppose that after a history, the set of active players is $S$.

(i) If $i$ is at the stage that he has to choose from the choice set $X(S) \times$ $\Theta(S)$, he chooses $\left(\hat{x}^{S}, \theta^{S}\right)$ where $\hat{x}^{S} \in C\left(v^{S}\right)$ and $\theta^{S}$ denotes an arbitrary permutation.

(ii) If $i$ is the proposer, he proposes $\left(S, \hat{x}^{S}\right)$.

(iii) For any status-quo $x^{S}$, if $i$ responds to some ongoing proposal $\left(T, y^{T}\right)$, he accepts it if and only if $y_{i}^{T} \geq \epsilon_{i} x_{i}^{S}+\left(1-\epsilon_{i}\right) u_{i}^{S}$, where $u_{i}^{S}$ is the payoff to $i$ when $i$ becomes the proposer and all players in $S$ implement this strategy profile.

Next, we show that the strategy profile described above is an SSPE. For any status quo $x^{S}$ and player $i \in S$ who is a respondent, clearly, he will accept any offer no less than $\epsilon_{i} x_{i}^{S}+\left(1-\epsilon_{i}\right) u_{i}^{S}$. This is because if he rejects, with $\epsilon_{i}$ probability he gets $x_{i}^{S}$ and with $1-\epsilon_{i}$ probability he becomes a new proposer and obtains $u_{i}^{S}$. So action (iii) cannot be improved upon. Then for any player $i$ who is the proposer, the maximum payoff he can get if he proposes a proposal that will certainly be accepted equals the optimal value of the following maximization problem:

$$
\max _{\substack{i \in T \subseteq S \\ y^{T} \in X(T)}}\left\{v(T)-\sum_{j \in T \backslash i} y_{j}^{T}\right\}
$$

$$
\text { s.t. } y_{j}^{T} \geq \epsilon_{j} x_{j}^{S}+\left(1-\epsilon_{j}\right) u_{j}^{S} \text {, for each } j \in T \text {. }
$$

Denote by $g_{i}\left(u^{S}\right)$ the maximum value so attained. It is easy to see that

$$
g_{i}\left(u^{S}\right)=\max _{i \in T \subseteq S}\left\{v(T)-\sum_{j \in T \backslash i}\left[\epsilon_{j} x_{j}^{S}+\left(1-\epsilon_{j}\right) u_{j}^{S}\right]\right\} .
$$


From Lemma 5.2, we know that (5.7) must have a fixed point $\hat{m}$ such that for each $i \in S$

$$
\hat{m}_{i}=\max _{i \in T \subseteq S}\left\{v(T)-\sum_{j \in T \backslash i}\left[\epsilon_{j} x_{j}^{S}+\left(1-\epsilon_{j}\right) \hat{m}_{j}\right]\right\} .
$$

Note that the current status-quo $\hat{x}^{S}$ is a core allocation, then by using Lemma 5.4 we get $u^{S}=\hat{m}^{S}=\hat{x}^{S}$. Considering the respondents' actions in (iii), if $i$ proposes $\left(S, \hat{x}^{S}\right)$, he will obtain the final payoff

$$
\begin{aligned}
& v(S)-\sum_{j \in S \backslash i}\left[\epsilon_{j} \hat{x}_{j}^{S}+\left(1-\epsilon_{j}\right) \hat{m}_{j}\right] \\
= & v(S)-\sum_{j \in S \backslash i} \hat{x}_{j}^{S} \\
= & \hat{x}_{i}^{S} .
\end{aligned}
$$

Let us now consider a deviation from the proposal $\left(S, \hat{x}^{S}\right)$. Given the acceptance strategies, any proposal $\left(T, x^{T}\right)$ that will certainly be accepted proposed by $i$ must satisfy that $x_{j}^{T} \geq \hat{x}_{j}^{S}$ for each $j \in S \backslash i$. Denote by $g_{i}$ the payoff for player $i$ in this case, we have

$$
g_{i}=v(T)-\sum_{j \in T \backslash i} x_{j}^{T} \leq v(T)-\sum_{j \in T \backslash i} \hat{x}_{j}^{S} \leq \hat{x}_{i}^{S},
$$

where the last inequality follows from $\hat{x}^{S} \in C\left(v^{S}\right)$. Now consider an unacceptable proposal $\left(T, x^{T}\right)$ proposed by $i$. Then there must exist some $j \in T$ such that $x_{j}^{T}<\hat{x}_{j}^{S}$. In this case, from the constructed actions (ii) and (iii), the expected payoff for $i$ when $j$ rejects his proposal equals $\epsilon_{i} \hat{x}_{i}^{S}+\left(1-\epsilon_{i}\right) \hat{x}_{i}^{S}=\hat{x}_{i}^{S}$. Therefore (ii) cannot be improved upon. Finally, we show that (i) forms a best move. Given the actions that players will follow in (iii) and (ii), the final payoff for each player $i$ will be $\hat{x}_{i}^{S}$ no matter who becomes the proposer in the bargaining process, which implies that no player can gain more payoff by changing his permutation choice. Besides, if some player chooses $x^{S} \neq \hat{x}^{S}$, the bargaining process will breakdown and his payoff will be no more than 0 . It is therefore obvious that if we implement the no-delay SSPE $\eta$ at the outset 
of the game, the equilibrium payoff profile is $x^{N} \in C(v)$.

We have seen in Theorem 5.1 that any core allocation of a totally balanced characteristic function game can be supported as an SSPE payoff. There is a natural question that immediately arises: Does every SSPE of a totally balanced characteristic function game implement a core allocation? The answer, which will be shown in the next theorem, is affirmative.

Theorem 5.5. Let $(N, v)$ be a characteristic function game and $G^{\epsilon}(N)$ the corresponding bargaining game. If $(N, v)$ is totally balanced, then for each $\epsilon \in(0,1)^{n}$, every SSPE payoff vector is a core element.

Proof. We proof this by contradiction. Let $\eta$ be an SSPE. Suppose that for some active player set $S$, the SSPE payoff vector for players in $S$ is not belong to $C\left(v^{S}\right)$. Let $r^{S}=\left(r_{i}^{S}\right)_{i \in S}$ be the SSPE payoff vector for players in $S,\left(x^{S \mid i}, \theta^{S \mid i}\right)$ the choice which is selected by $i \in S$ in the first stage, $\theta^{S}$ the composition of $\left(\theta^{S \mid i}\right)_{i \in S}$. Then we have the following claims:

Claim 1. $x^{S \mid i}=x^{S \mid j}=x^{S}$ for any $i, j \in S$.

Suppose not. Then player $\theta^{S}(1)$ will receive $d<0$. This player can certainly change his permutation choice such that he is not the first one with respect to the new composition of permutations. In this way, he will get 0 instead of $d$. This contradicts the fact that $\eta$ is an SSPE.

Claim 2. For each $i \in S, r_{i}^{S} \geq u_{i}^{S}$.

If $i=\theta^{S}(1)$, it is obvious that $r_{i}^{S}=u_{i}^{S}$. Suppose $r_{k}^{S}<u_{k}^{S}$ for some player $k \neq \theta^{S}(1)$. Then $k$ can change his permutation choice in the first stage to make sure that he becomes the first proposer. In this case, $k$ will receive $u_{k}^{S}>r_{k}^{S}$, which is in contradiction with the hypothesis that $\eta$ is an SSPE.

Claim 3. For each $i \in S, r_{i}^{S} \geq x_{i}^{S}$.

This is a natural result since any player $i \in S$ can simply reject any proposal and propose any offer that will certainly be rejected just to receive $x_{i}^{S}$. Thus the SSPE payoff for $i \in S$ can not be less than $x_{i}^{S}$. 
Claim 4. For each $j \in S$ who is a respondent and has to respond to the statusquo $x^{S}$ and a proposal $\left(T, y^{T}\right)$, he will accept if and only if $y_{j}^{T}>\epsilon_{j} x_{j}^{S}+(1-$ $\left.\epsilon_{j}\right) u_{j}^{S}$.

This is a straightforward consequence of an SSPE. Since if he rejects, his expect payoff is $\epsilon_{j} x_{j}^{S}+\left(1-\epsilon_{j}\right) u_{j}^{S}$, he must accept any proposal that is better than $\epsilon_{j} x_{j}^{S}+\left(1-\epsilon_{j}\right) u_{j}^{S}$.

Since $(N, v)$ is totally balanced, $(N, v)$ must be superadditive. This implies that $r^{S} \in X(S)$. Because $r^{S} \notin C\left(v^{S}\right)$, there must be a $T \subset S$ such that $v(T)>$ $\sum_{i \in T} r_{i}^{S}$. Then there must be a $z^{T} \in X(T)$ such that $\sum_{i \in T} z_{i}^{T}=v(T)$ and $z_{i}^{T}>$ $r_{i}^{S}$ for each $i \in T$. Hence, we have for each $i \in T$

$$
z_{i}^{T}>r_{i}^{S} \geq \epsilon_{i} x_{i}^{S}+\left(1-\epsilon_{i}\right) u_{i}^{S},
$$

where the second inequality follows from Claim 2 and Claim 3. If some player $k \in T$ changes his permutation choice in the first stage to becoming the first proposer and proposes $\left(T, z^{T}\right)$, from Claim 4 we know that this proposal will be accepted by all players in $T$. Then for each player $i \in T$, the final payoff for $i$ will be $z_{i}^{T}$ larger than $r_{i}^{S}$, which contradicts the hypothesis that $\eta$ is an SSPE.

Combining the above two theorems, we can obtain our main result.

Theorem 5.6. Let $(N, v)$ be a characteristic function game and $G^{\epsilon}(N)$ the corresponding bargaining game. If $(N, v)$ is totally balanced, then for each $\epsilon \in(0,1)^{n}$, the set of SSPE payoff vectors coincide with the core of $(N, v)$.

\subsection{Discussion}

\subsubsection{The implementation of the coalition structure core}

It should be noted that our bargaining game requires totally balancedness of the characteristic function game for the implementation of the core in SSPE. The totally balancedness also implies the superadditive structure of the game, that is, the grand coalition can always form. However, in most of the economic situations, the core can be empty and the coalition formation does not 
have to be superadditive. In this case, it is natural to consider the coalition structure core [34]. Let $\mathscr{P}$ be the set of all partitions ${ }^{2}$ of $N$. Define

$$
X(\mathscr{P})=\bigcup_{P \in \mathscr{P}} X(P)
$$

where

$$
X(P)=\left\{x \in \mathbb{R}^{n} \mid \sum_{i \in S} x_{i} \leq v(S) \text { for each } S \in P\right\} .
$$

The coalition structure core of a characteristic function game $(N, v)$ is defined by

$$
\hat{C}(v)=\left\{x \in X(\mathscr{P}) \mid \sum_{i \in S} x_{i} \geq v(S) \text { for all } S \subseteq N\right\} .
$$

Serrano et al. [77] argue that with a minor modification, their noncooperative bargaining game, which is originally designed for the implementation of the core, can also implement the coalition structure core in subgame perfect equilibrium. This makes us wonder whether our mechanism can get the same result by some extension. The answer is affirmative. Let $\mathscr{P}_{N^{t}}$ be the set of all partitions of the active player set $N^{t}$. It is easy to verify that if the choice set is changed from $X\left(N^{t}\right) \times \Theta\left(N^{t}\right)$ to $X\left(\mathscr{P}_{N^{t}}\right) \times \Theta\left(N^{t}\right)$ in each period and the feasible allocations for a proposer to choose from extending to $X\left(\mathscr{P}_{S}\right)$ when he wants to propose $S$, the modified mechanism implements the coalition structure core in SSPE if for each $S \subseteq N, \hat{C}\left(v^{S}\right)$ is nonempty. The proof for this result is almost identical to the proof provided to show the implementation of the core, thus can be neglected here.

\subsubsection{No-delay SSPE}

One of the most interesting concepts in the literature on bargaining games that have been widely studied is the no-delay SSPE. We have already seen in Theorem 5.1 that no-delay SSPEs exist if $(N, v)$ is totally balanced. In this subsection, we further investigate the no-delay SSPEs in general characteristic function games.

\footnotetext{
${ }^{2}$ A partition of $N$ is defined by $P=\left\{S_{1}, \ldots, S_{k}\right\}$, where $1 \leq k \leq n, S_{i} \neq \emptyset$ for $i=1, \ldots, k$, $S_{i} \cap S_{j}=\emptyset$ for $i, j=1, \ldots, k(i \neq j)$, and $\bigcup_{i=1}^{k} S_{i}=N$.
} 
Theorem 5.7. Let $\eta$ be a no-delay SSPE and $x^{S}$ the status-quo when the active player set is $S$. Then for each active player set $S$ and each $i \in S$, we have

$$
u_{i}^{S}=\max _{i \in T \subseteq S}\left\{v(T)-\sum_{j \in T \backslash i}\left[\epsilon_{j} x_{j}^{S}+\left(1-\epsilon_{j}\right) u_{j}^{S}\right]\right\} .
$$

Proof. Since $\eta$ is an SSPE, for any history that ends with the active player set $S$, if any player $i \in S$ has to make a proposal, he can guarantee his equilibrium payoff $u_{i}^{S}$. This implies that any player $i \in S$ who is a respondent, he will accept the offer no less than $\epsilon_{i} x_{i}^{S}+\left(1-\epsilon_{i}\right) u_{i}^{S}$. To see why, if he rejects, with $\epsilon_{i}$ probability he gets $x_{i}^{S}$ and with $1-\epsilon$ probability he becomes a new proposer and obtains his equilibrium payoff $u_{i}^{S}$. Therefore $\epsilon_{i} x_{i}^{S}+\left(1-\epsilon_{i}\right) u_{i}^{S}$ can be seen as the expected payoff for player $i$ when he rejects some ongoing proposal. It follows that if some proposer $i$ wants to make a proposal that will certainly be accepted, the maximum payoff he can guarantee for himself is

$$
w_{i}^{S}=\max _{i \in T \subseteq S}\left\{v(T)-\sum_{j \in T \backslash i}\left[\epsilon_{j} x_{j}^{S}+\left(1-\epsilon_{j}\right) u_{j}^{S}\right]\right\} .
$$

From the definition of $u_{i}^{S}$, we must have $u_{i}^{S} \geq w_{i}^{S}$. However, the strict inequality $u_{i}^{S}>w_{i}^{S}$ can not be true, since $\eta$ is a no-delay stationary strategy. Hence we obtain (5.9).

Theorem 5.7 provides a necessary condition for an SSPE to be no-delay. However, it does not mean that if $u^{S}$ satisfies the equation (5.9), the corresponding stationary strategy profile has to be a no-delay SSPE. The following example states this fact.

Example 5.1. Consider a three-person bargaining game $G^{\epsilon}(N)$, where $\epsilon_{1}=$ $\epsilon_{2}=\epsilon_{3}=\hat{\epsilon}, N=\{1,2,3\}, v(\{1,2\})=v(\{1,3\})=v(\{2,3\})=100, v(S)=0$ for all other $S \subseteq N$.

We first construct a strategy profile $\eta$ as follows:

Suppose that after a history, the set of active players is $S$. 
(i) At Stage 1, every player chooses $\left(x^{S}, \theta^{S}\right)$ where for each $i \in S, x_{i}^{S}=\frac{\nu(S)}{S}$; $\theta^{S}=(1,2,3)$ if $S=N ; \theta^{S}=(1,2)$ if $S=\{1,2\} ; \theta^{S}=(1,3)$ if $S=\{1,3\}$ and $\theta^{S}=(2,3)$ if $S=\{2,3\}$

(ii) At stage 2,

- if player 1 is the proposer, he chooses to propose $\left(\{1,2\},\left(\frac{100}{2-\hat{\epsilon}}, \frac{100(1-\hat{\epsilon})}{2-\hat{\epsilon}}\right)\right)$ if $S=N$, and $(S,(50,50))$ if $s=2$.

- If player 2 is the proposer, he chooses to propose $\left(\{2,3\},\left(\frac{100}{2-\hat{\epsilon}}, \frac{100(1-\hat{\epsilon})}{2-\hat{\epsilon}}\right)\right)$ if $S=N$, and $(S,(50,50))$ if $s=2$.

- If player 3 is the proposer, he chooses to propose $\left(\{1,3\},\left(\frac{100}{2-\hat{\epsilon}}, \frac{100(1-\hat{\epsilon})}{2-\hat{\epsilon}}\right)\right)$ if $S=N$, and $(S,(50,50))$ if $s=2$.

- Any respondent only accepts the offer greater than $\frac{100(1-\hat{\epsilon})}{2-\hat{\epsilon}}$ if $S=N$, and 50 if $s=2$.

We can clearly see that the strategy profile constructed above is no-delay and $u_{i}^{N}=\frac{100}{2-\hat{\epsilon}}$ for each $i \in N, u_{i}^{S}=50$ for each $i \in S$ when $s=2$. It is easy to verify that for each $S, u^{S}$ satisfies the equation (6.1). However, we claim that $\eta$ is not an SSPE. Note that if $\eta$ is implemented, the first status-quo is $(0,0,0)$ and the endogenous protocol is $(1,2,3)$. So player 1 will become the proposer and offer $\frac{100(1-\hat{\epsilon})}{2-\hat{\epsilon}}$ to player 2 , who will certainly accept the offer. Then coalition $\{1,2\}$ leaves the game. In this case, player 3 has to work alone and obtains 0 . But if player 3 adjusts his strategy at Stage 1 by changing his permutation from $(1,2,3)$ to $(3,1,2)$, he will become the first proposer in this game and obtain $\frac{100}{2-\hat{\epsilon}}$. We can also notice that player 2 has the same motivation to deviate since $\frac{100}{2-\hat{\epsilon}}>\frac{100(1-\hat{\epsilon})}{2-\hat{\epsilon}}$. Hence, $\eta$ is not an SSPE. In fact, this example does not even yield any SSPE, since any player, who finds that he will end up in a disadvantageous position in the game, is apt to change his permutation strategy at Stage 1 in order to reach a better position. This example also illustrates the fact that the existence of SSPEs can not be guaranteed if $(N, v)$ is not totally balanced.

The next theorem provides a sufficient condition for the existence of nodelay SSPEs. It shows that if the equilibrium payoff for the very first proposer at Stage 2 in each period is strictly larger than his allocation in the current status-quo, the corresponding SSPE is no-delay. 
Theorem 5.8. Given any SSPE $\eta$ and active player set $S$, let $x^{S}$ be the status$q u o, k$ the first proposer at the beginning of Stage 2 when $\eta$ is implemented. If $u_{k}^{S}>x_{k}^{S}, \eta$ is a no-delay SSPE.

Proof. Suppose that SSPE $\eta$ is not no-delay. Then there must exist an active player set $S$ in which the first proposer $k$ proposes an unacceptable offer to some player, say $l$, and then $l$ rejects it. Let $r^{S}=\left(r_{i}^{S}\right)_{i \in S}$ be the corresponding SSPE payoff vector, $b_{i j}$ the conditional payoff for $i$ in SSPE $\eta$, given that $j$ becomes the proposer in the active player set $S$. Since $k$ proposes an offer that will certainly rejected by $l$, we must have $u_{k}^{S}=\epsilon_{k} x_{k}+\left(1-\epsilon_{k}\right) b_{k l}$. Then we make the following claim:

Claim 5. For any $i, j \in S, r_{i}^{S} \geq b_{i j}$.

The proof of this claim is similar to that of Claim 2 in Theorem 5.5. Suppose for some $i, j \in S, r_{i}^{S}<b_{i j}$. Then $i$ can change his permutation choice at Stage 1 to make sure that $j$ becomes the first proposer. In this case, $i$ will receive $b_{i j}>r_{i}^{S}$, which is in contradiction with the hypothesis that $\eta$ is an SSPE.

Then by using Claim 3 and Claim 5 we have

$$
r_{k}^{S} \geq \epsilon_{k} x_{k}^{S}+\left(1-\epsilon_{k}\right) b_{k l}=u_{k}^{S} .
$$

On the other hand, since player $k$ is the first proposer in the active player set $S$ when $\eta$ is implemented, we have $r_{k}^{S}=u_{k}^{S}$. This implies that $u_{k}^{S}=x_{k}^{S}$, a contradiction. It should be noted that $u_{k}^{S}$ can not strictly less than $x_{k}^{S}$, which can be seen from the fact that $r_{k}^{S}=u_{k}^{S}$ and Claim 3 .

\subsubsection{Related studies and Comparisons}

Serrano et al. [77] introduce a one-period ${ }^{3}$ bargaining game which implements the core allocations of economic environments in subgame perfect equilibrium. The noncooperative bargaining game provided in their work incorporate the same proposer-selecting method as we do at Stage 1. That is,

\footnotetext{
${ }^{3}$ That is, the whole bargaining game will stop whether the proposal is unanimously accepted or rejected by some player.
} 
the bargaining protocol is determined by the composition of permutations of players, and the game can not proceed to Stage 2 unless a unanimous agreement on a feasible allocation is reached. The main difference is that after the rejection of a proposal, in [77], the game terminates and the status-quo is implemented for all players with certainty; while in our game, only the players in the coalition selected by the proposer are facing the risk of receiving the payoff in the status-quo. Moreover, the first rejector may become the new proposer with some probability. We can clearly see that when $\epsilon_{i}$ goes to 1 for each $i \in N$, our noncooperative bargaining game is almost the same as theirs (our bargaining game may involve multiple periods). Therefore, we can obtain our next theorem, the proof of which is almost identical to that of Theorem 5.1 in [77] and shall be omitted here.

Theorem 5.9. Let $(N, v)$ be a characteristic function game and $G^{\epsilon}(N)$ the corresponding bargaining game. If $(N, v)$ is totally balanced and $\epsilon_{i}=1$ for each $i \in N$, then the set of SSPE payoff vectors coincide with the core of $(N, v)$.

It remains to show what will happen if we let $\epsilon_{i}$ goes to 0 for each $i \in N$. If that is the case, apparently, the strategic behavior of players at Stage 2 will barely be affected by the status-quo determined at Stage 1. Additionally, our bargaining game will be similar to that in [15], except that the bargaining protocol is endogenously determined by the strategies of players in every period and the discounting factor is not involved. Then it is easy to verify that in any subgame $S$, any $y^{S} \in C\left(v^{S}\right)$ is a fixed point of the function $\phi$. By using the same proof methods as that being used in Theorem 5.1 and Theorem 5.5, the following result can be easily obtained.

Theorem 5.10. Let $(N, v)$ be a characteristic function game and $G^{\epsilon}(N)$ the corresponding bargaining game. If $(N, v)$ is totally balanced and $\epsilon_{i}=0$ for each $i \in N$, then the set of SSPE payoff vectors coincide with the core of $(N, v)$. 


\section{Chapter 6}

\section{A coalitional bargaining game with majority rule}

This chapter proposes a noncooperative bargaining model in which each player has several votes and the approval of a proposal requires only a fraction of the approval committees' votes. We first provide the characterization of the set of stationary subgame perfect equilibria. Then with the help of this characterization, we show the existence of these equilibria. Moreover, when we restrict our attention to the grand-coalition efficient games, we show that the pivotal players extract all the gains from the grand coalition if their discount rates go to 1 . Finally, we show the existence of grand-coalition efficient games.

\subsection{Introduction}

Since the seminal work of Rubinstein [75], noncooperative bargaining games have received more and more attention in the literature on dynamic collective decision-making. In these games, a group of players negotiates over the allocation of the grand coalition. The negotiation starts with a proposer being selected by a predetermined protocol to propose a proposal, which can only be implemented by an approval committee. If the proposal is approved by the approval committee then the agreement is implemented; otherwise, another 
proposer is selected by the protocol. The approval committee here can be the set of all players in the game, or some set of players contained in the content of the proposal, depends on the model.

Based on whether the grand coalition is the only object in which proposers intend to reallocate resources, noncooperative games of dynamic bargaining can be divided into two types: coalitional bargaining games and legislative bargaining games. In coalitional bargaining games, a proposal contains a coalition and a feasible payoff distribution for the coalition. The implementation of the proposal needs unanimous consent within that coalition. See [7], [15], [33], [46], [68], and [73], among others. While in legislative bargaining games, a proposal can only be the allocation of a fixed cake which is the worth of the grand coalition, and the agreement also requires the unanimous consent of all players. See for example, [3], [27], [67], and [82]. Compared to coalitional bargaining games, legislative bargaining games have another distinct feature which is that the implementation of a proposal needs only a majority votes in favor of the proposal.

In this chapter, we consider a sequential bargaining game in which the approval of a proposal requires that the votes of players who are in favor of the proposal meet a quota. The model can be stated as follows: the game may take an infinite amount of periods. Each player has several votes. At the beginning of each period, a proposer is randomly selected among the current active players. The random selection of proposers is exogenously given by the model. Then, the proposer chooses a coalition, which is a subset of the current active player set and must contain himself, and a payoff to the members of that coalition. Then all the members of the coalition, the respondents, vote yes or no for the proposal. If the total number of 'yes' votes is at least some given quota for the coalition, the proposal is implemented. Then the players in the proposed coalition get their payoff from the proposer and leave the game. The remaining players stay active; otherwise, nothing changes, and a new proposer is randomly selected. The infinite horizon game we describe above is an extension of the model introduced by Okada [68, 69], who studied a similar game in which each player only has one vote and the approval of a proposal needs the unanimous consent of all players in the proposed coalition.

We first show the characterizations for the strategies and the expected 
payoffs of the stationary subgame perfect equilibria (SSPE), and then the existence of SSPEs is established. Then, we restrict our attention to the grandcoalition efficient games, in which the grand coalition will always be proposed in any SSPE, independent of the proposer. In this case, our coalitional bargaining games will reduce to the classical legislative bargaining situations which are described in [82] and [58]. Snyder et al. [82] use a distributive bargaining model where the set of players is infinite, and they show that expected equilibrium payoffs are proportional to the votes. Montero [58] provides a necessary and sufficient condition for expected equilibrium payoffs to be proportional to voting weights in finite legislative bargaining games. Different from those papers, this chapter concerns itself with the exploration of the effect of veto power in legislative bargaining. Our main result shows that the pivotal players, whose votes are essential for the implementation of any SSPE proposal, extract all the payoffs of the grand coalition when their discount rates go to 1 . This result is almost identical to the study of Winter [89], which shows that the worth of the grand coalition is divided between the veto players and each nonveto player earns zero payoff by using a completely different noncooperative mechanism from ours.

This chapter is organized as follows. In the next section, we introduce our coalitional bargaining model. Section 6.3 provides the characterizations of strategies and payoffs of SSPEs, and the existence of SSPEs. Section 6.4 concentrates on the grand-coalition efficient games. Section 6.5 is devoted to showing the existence of grand-coalition efficient games.

\subsection{The model}

The underlying cooperative game is a pair $(N, v)$, where $N=\{1, \ldots, n\}$ is the set of players, $v$ is a characteristic function that assigns to every coalition $S \subseteq$ $N$ a real value $v(S)$. The lowercase $s$ denotes the cardinality of a coalition $S$. In this chapter, we assume that $(N, v)$ is $(0,1)$-normalized and superadditive. Every player $i$ has an integer number of votes $w_{i}$ with $w_{i} \geq 1$. Each coalition $S$ has a quota $q_{S}$ with $0<q_{S} \leq \sum_{i \in S} w_{i}$. A payoff for a player $i$ can be denoted 
by a real number $x_{i}$. A feasible payoff set for any coalition $S \subseteq N$ is defined by

$$
X(S)=\left\{x^{S} \in \mathbb{R}^{S} \mid \sum_{i \in S} x_{i}^{S}=v(S) \text { and } x_{i} \geq 0 \text { for any } i \in S\right\} .
$$

We now formally describe our bargaining model. The negotiation may take an infinite amount of periods. Let $t$ be the period index and $N^{t}$ be the set of players that still active in period $t$. Set $N^{1}=N$. Let $p=\left(p_{i}^{S}\right)_{\substack{S \subseteq N \\ i \in N}}$ be the proposer probabilities. In each period, a proposer $i \in N^{t}$ is randomly selected with probability $p_{i}^{N^{t}}$. A feasible proposal for a proposer $i$ in period $t$ is a pair $\left(S, x^{S}\right)$ where $S \subseteq N^{t}, i \in S$ and $x^{S} \in X(S)$. We denote by $\mathscr{F}_{i}^{t}$ the set of all feasible proposals for $i$ in period $t$. Then proposer $i$ chooses a proposal $\left(S, x^{S}\right) \in \mathscr{F}_{i}^{t}$. The offer $x_{j}^{S}$ to the player $j \in S$ can be thought of as the bribe used for buying the votes of $j$. All players in $S$ vote yes or no for the proposal. It is naturally assumed that the proposer himself always votes yes. If the sum of the votes of players who vote yes is at least $q_{S}$, then the proposal is approved and all members in $S$ leave the game. Thereafter, the active player set changes to $N^{t+1}=N^{t} \backslash S$, and the game proceeds. Otherwise, the active player set does not change and the game is repeated with a new proposer being selected randomly by using the same procedure.

All players in our model are assumed to discount their future payoffs at rates between 0 and 1. Formally, if a player $i$ receives a payoff $x_{i}^{S}$ in period $t$, his discounted present payoff is $\left(\delta_{i}\right)^{t-1} x_{i}^{S}$, where $\delta_{i} \in(0,1)$ and $\delta_{i}$ is called the discount factor for player $i$. If a perpetual disagreement prevails, the remaining players who have not achieved agreements receive zero payoffs. The game is called a coalition bargaining game with majority rule (CBGMR). We define the subgame $G(S)$ as the game starting from the period when $S$ is an active player set.

In order to describe players' strategies as well as equilibria, we need to introduce a bit of notations. Let $h^{t}$ be the history up to period $t$ (including $t$ ), which contains all information about all proposers, the proposals of proposers and the decisions of the respondents before the start of period $t+1$. Whenever $i$ is selected as the proposer in period $t+1$, we denote by $h^{t+1}(i)$ the history which includes $h^{t}$ and the history up to $i$ being selected. Similarly, 
whenever a proposer $i$ makes a proposal $\left(S, x^{S}\right)$ in period $t+1$, we denote by $h^{t+1}\left(i, S, x^{S}\right)$ the history which includes $h^{t}$ and the history up to when proposer $i$ makes that proposal. For any history $h$, we represent by $\eta_{i}(h)$ the behavior strategy for player $i$, which specifies the action of player $i$ whenever it is i's turn to make a decision. Given any set $A$, let $\Delta(A)$ be the set of probability measures on $A$. If $i$ is a selected proposer in period $t$ and $h^{t}(i)$ is the corresponding history, $\eta_{i}\left(h^{t}(i)\right) \in \Delta\left(\mathscr{F}_{i}^{t}\right)$ specifies the mixed feasible proposal offered by $i$ in period $t$. If $i$ is a respondent in $S$ and about to respond the proposal $\left(S, x^{S}\right)$ offered by $j$ in period $t$, and $h^{t}\left(j, S, x^{S}\right)$ is the corresponding history, $\eta_{i}\left(h^{t}\left(j, S, x^{S}\right)\right) \in \Delta(\{$ yes, no $\})$ denotes the mixed decision made by $i$ in period $t$. Let $\eta_{i}$ be the strategy of player $i$ in this game. The strategy of player $i$ includes instructions on all behavior strategies whenever it is $i$ 's turn to make a decision.

In this chapter, we look for stationary subgame perfect equilibrium (SSPE). A strategy profile $\eta=\left(\eta_{1}, \cdots, \eta_{n}\right)$ is called stationary if the corresponding behavior strategies of all players only depend on the current active player set $N^{t}$, the offer proposed by the proposer in the current period $t$ (if any), but not on other aspects of the history $h^{t-1}$ of the game. A strategy profile $\eta$ is called subgame perfect if for any $i \in N, \eta_{i}$ is a best response strategy for $i$ after every history of play.

Definition 6.1. A strategy profile $\eta=\left(\eta_{1}, \cdots, \eta_{n}\right)$ is called stationary subgame perfect equilibrium if $\eta$ is stationary and subgame perfect.

\subsection{The characterization and existence of SSPEs}

In this section, we first characterize the strategies and the expected payoffs of SSPEs, and then provide a proof of their existence. Given that we focus on the stationary strategies, if there is no danger of confusion we denote $\eta_{i}^{S}\left(T, x^{T}\right)$ as the probability that proposer $i$ chooses the proposal $\left(T, x^{T}\right)$ when the current active player set is $S$. We also denote $\eta_{i}^{S}\left(j, T, x^{T}\right.$, yes $)$ as the probability that respondent $i$ decides to accept the offer $\left(T, x^{T}\right)$ proposed by $j$. Given any SSPE $\eta$, let $u^{S}=\left(u_{i}^{S}\right)_{i \in S}$ be the expected equilibrium payoffs for $S$ when $S$ is the active player set. For notational convenience, we write $u=\left(u_{i}\right)_{i \in N}$ instead 
of $u^{N}=\left(u_{i}^{N}\right)_{i \in N}$ when we consider the grand coalition. For each coalition $S$ with $i \in S$, we define the $s-1$ dimensional incidence vector of a subset $T$ of $S$ for proposer $i$ as $\gamma^{i, S}(T)$, such that $\gamma_{j}^{i, S}(T)=1$ for each $j \in T$, and $\gamma_{j}^{i, S}(T)=0$ for each $j \in S \backslash T$. This incidence vector indicates the players in the proposed coalition who will be bribed by the proposer. Finally, the set of all incidence vectors for coalition $S$ and $i \in S$ is denoted by $\Gamma^{i, S}=\left\{\gamma^{i, S}(T) \mid T \subseteq S\right\}$.

Before we provide the proposition which characterizes SSPEs, some definitions and assumptions have to be introduced.

Definition 6.2. For any CBGMR, a player $i \in N$ is called pivotal for the game if for any SSPE proposal $\left(S, x^{S}\right) \in \mathscr{F}_{j}^{t}$ made by any player $j$ in any period $t$ such that $i \in S,\left(S, x^{S}\right)$ cannot be implemented if $i$ votes no for it.

Definition 6.3. For any CBGMR, a player $i \in N$ is called dispensable for the game if for any SSPE proposal $\left(S, x^{S}\right) \in \mathscr{F}_{j}^{t}$ made by any player $j$ in any period $t$ such that $i \in S$, the implementation of $\left(S, x^{S}\right)$ has no relevance to whether $i$ votes yes or no for it.

Note that a pivotal player has a key role in the implementation of any SSPE proposal in which the coalition contains himself. An SSPE proposal will surely be denied if a pivotal player votes against it. For another extreme, the decision of a dispensable player is of no account with the final outcome. Generally speaking, pivotal players and dispensable players represent two opposite sides of the voting power.

We impose the following assumption throughout to establish a tie-breaking rule for the behavior strategies chosen by the respondents.

Assumption 1. (i) The respondents who are indifferent between the proposal being accepted and moving to the next period vote yes for the proposal.

(ii) All respondents act as if they are pivotal players.

The first assumption of Assumption 1 excludes some trivial SSPEs, for example when all players always vote no when they are indifferent between the proposal being accepted and moving to the next period. The second assumption of Assumption 1 is natural as every respondent would expect his or her desirable payoff for his own benefit and assume that he or she is a pivotal 
player. The following proposition provides a characterization of the SSPEs (under Assumption 1).

Proposition 6.1. Let $\eta$ be a strategy profile, and $\left(z^{S}\right)_{S \subseteq N}$ the expected payoff vectors corresponding to $\eta$. Strategy profile $\eta$ is an SSPE and $\left(z^{S}\right)_{S \subseteq N}$ are the corresponding expected equilibrium payoff vectors if and only if $\eta$ satisfies,

(i) if $i$ is a proposer and $\eta_{i}^{S}\left(\hat{T}, x^{\hat{T}}\right)>0$, then there is a $\gamma_{j}^{i, \hat{T}}(\hat{R})$ such that $x_{j}^{\hat{T}}=\delta_{j} z_{j}^{S} \gamma_{j}^{i, \hat{T}}(\hat{R})$, and $\hat{T}, \gamma^{i, \hat{T}}(\hat{R})$ solve the following program

$$
\begin{array}{ll}
\max _{\substack{T \subseteq S_{\text {s.t. }} \subseteq \in T \\
R \subseteq T}} & v(T)-\sum_{j \in T \backslash i} \delta_{j} z_{j}^{S} \gamma_{j}^{i, T}(R) \\
\text { subject to } & w_{i}+\sum_{j \in T \backslash i} w_{j} \gamma_{j}^{i, T}(R) \geq q_{T},
\end{array}
$$

(ii) if $j$ is a respondent, $\eta_{j}^{S}\left(i, T, x^{T}\right.$,yes $)=1$ if $x_{j}^{T} \geq \delta_{j} z_{j}^{S}$.

Proof. (If.) We first show that the behavior strategy (ii) cannot be improved upon, which means that by deviating (ii), any respondent will not be better off. By definition, the expected payoff for any respondent $j$ is $\delta_{j} z_{j}^{S}$ if the proposal is not approved. The rejection of the proposal will lead to the expected payoffs $\delta_{j} z_{j}^{S}$ for each respondent $j$. Therefore, any respondent $j$ will certainly accept any offer to him which is larger than $\delta_{j} z_{j}^{S}$. This implies that the behavior strategy (ii) cannot be improved upon.

We now show that the behavior strategy (i) cannot be improved upon. Given any proposer $i$ and the coalition $T$ proposed by $i$, since $i$ will get the remainder of $v(T)$ after his proposal is accepted, his best option is offering each player that he bribes exactly the amount $\delta_{j} z_{j}^{S}$. Clearly, among the proposals which will be accepted by respondents, the proposal maximizing the payoff of proposer $i$ can be derived from the program (6.1). The constraint $w_{i}+\sum_{j \in T \backslash i} w_{j} \gamma_{j}^{i, T}(R) \geq q_{T}$ makes sure that the proposal will be approved. We next show that any behavior strategy of choosing any proposal always being rejected by respondents will decrease the expected payoff of a proposer. If $i$ chooses any proposal which will always be rejected, his proposal will be rejected and the continuation payoff for $i$ is $\delta_{i} u_{i}^{S}$. Let $m_{i}^{S}$ be the maximal value of (6.1), and $\mathbf{1}^{s-1}$ be an $s-1$ dimensional vector such that $\mathbf{1}_{j}^{s-1}=1$ 
for any $j \in S \backslash i$. Note that for each $\gamma^{i, S}(T) \in \Gamma^{i, S}$

$$
v(S)-\sum_{j \in S \backslash i} \delta_{j} z_{j}^{S} \gamma_{j}^{i, S}(T) \geq v(S)-\sum_{j \in S \backslash i} \delta_{j} z_{j}^{S} \mathbf{1}_{j}^{s-1},
$$

the pair $\left(S, \mathbf{1}^{s-1}\right)$ must be a feasible solution of the program (6.1). Thus we have

$$
m_{i}^{S} \geq v(S)-\sum_{j \in S \backslash i} \delta_{j} z_{j}^{S} \mathbf{1}_{j}^{s-1}>v(S)-\sum_{j \in S \backslash i} z_{j}^{S} \geq z_{i}^{S},
$$

where the last inequality follows from the superadditivity of characteristic function $v$. Hence, $m_{i}^{S}>z_{i}^{S}>\delta_{i} z_{i}^{S}$.

(Only if.) Clearly, the fact that the behavior strategy (ii) is the only best response for each respondent from Assumption 1. From (If.) part, we have already seen that choosing any proposal always being rejected by respondents is not a best response for each proposer. Thus, the only best response for each proposer is to choose proposals that satisfy (i).

Note that given any SSPE $\eta$ and any active player set $S$, an SSPE proposal $\left(T, x^{T}\right)$ made by player $i$ can be uniquely represented by proposals in the form of $\left(T, \gamma^{i, T}(R)\right)$ which solve the program (6.1). To simplify notation we use $\eta_{i}^{S}\left(T, \gamma^{i, T}(R)\right)$ to denote the probability that the proposer $i$ chooses the proposal $\left(T, x^{T}\right)$ where $x_{j}^{T}=\delta_{j} u_{j}^{S} \gamma_{j}^{i, T}(R)$ for any $j \in T \backslash i$. The following proposition characterizes the equilibrium payoffs of SSPE.

Proposition 6.2. Let $\eta$ be an SSPE. For any $i \in S$, let $\left(T_{i}, \gamma^{i, T_{i}}\left(R_{i}\right)\right)$ be an SSPE proposal of player $i$, and $\mathscr{F}_{i}^{S}$ be the set of all SSPE proposals of player $i$. The expected equilibrium payoff $u_{i}^{S}$ satisfies

$$
\begin{aligned}
u_{i}^{S}=p_{i}^{S} & \left(v\left(T_{i}\right)-\sum_{j \in T_{i} \backslash i} \delta_{j} u_{j}^{S} \gamma_{j}^{i, T_{i}}\left(R_{i}\right)\right) \\
& +\sum_{j \in S \backslash i} \sum_{\left(T_{j}, \gamma^{j, T_{j}}\left(R_{j}\right)\right) \in \mathscr{F}_{j}^{S}} p_{j}^{S} \eta_{j}^{S}\left(T_{j}, \gamma^{j, T_{j}}\left(R_{j}\right)\right) Z_{i}\left(T_{j}\right),
\end{aligned}
$$


where

$$
Z_{i}\left(T_{j}\right)= \begin{cases}\delta_{i} u_{i}^{S} \gamma_{i}^{j, T_{j}}\left(R_{j}\right) & \text { if } i \in T_{j}, \\ \delta_{i} u_{i}^{S \backslash T_{j}} & \text { otherwise. }\end{cases}
$$

Proof. By Proposition 6.1, we know that the SSPE strategy for a proposer is to choose the proposals which solve the program (6.1) with positive probabilities. Hence, at the beginning of period $t$, with probability $p_{i}^{S}$ player $i$ is selected as a proposer, in which case the payoff to $i$ is $v\left(T_{i}\right)-\sum_{j \in T_{i} \backslash i} \delta_{j} u_{j}^{T} \gamma_{j}^{i, T_{i}}\left(R_{i}\right)$. With probability $p_{j}^{S}$, player $j \in S \backslash\{i\}$ is selected as a proposer, in which case the expected payoff to $i$ is dependent on whether $i$ is included in the coalition proposed by $j$. Note that $\eta_{j}^{S}\left(T_{j}, \gamma^{j, T_{j}}\left(R_{j}\right)\right)$ is the probability that $j$ chooses the pair $\left(T_{j}, \gamma^{j, T_{j}}\left(R_{j}\right)\right)$ which solves the program (6.1), we can see that the value $\sum_{\left(T_{j}, \gamma^{j, T_{j}}\left(R_{j}\right) \in \mathscr{F}_{j}^{S}\right.} p_{j}^{S} \eta_{j}\left(T_{j}, \gamma^{j, T_{j}}\left(R_{j}\right)\right) Z_{i}\left(T_{j}\right)$ is the expected payoff to $i$ when $j$ is selected as a proposer. Thus, the right hand side of (6.2) provides the expected payoff to player $i$ at the beginning of current period before the proposer is selected.

We next show that an SSPE exists for every CBGMR. The proof of the following theorem uses a similar technique to the one used in [73].

Theorem 6.1. There exists at least one SSPE for every CBGMR.

Proof. We use an inductive proof on the cardinality of the active player set. We first provide a brief sketch of this proof: (i) for one player, SSPEs always exist. (ii) Suppose an SSPE exists for each game with less than $n$ players. For each subgame $G(S)$ with less than $n$ players, we fix an SSPE and generate the corresponding expected equilibrium payoff profile $u^{S}$. We construct the equilibrium strategies by defining a correspondence from the space of pairs of payoff vectors and strategy vector. These payoff vectors represent the expected payoff of the players, while the strategy vectors represent the strategies of proposers. The mapping is as follows, for a pair of payoff and strategy vectors, compute for each player a best response to those strategies and expected payoffs of the other players. We show that there is a fixed point which contains a payoff profile for $N$ and also a strategy profile for all possible proposers. (iii) We then construct an SSPE $\eta$ by this fixed point. 
We now formally describe our proof. Let $\alpha_{i}$ be a strategy of proposer $i$, and $A_{i}$ be the strategy space of $\alpha_{i}$. Define $A=\prod_{i \in N} A_{i}$, and $X$ as the payoff vector space in $\mathbb{R}_{+}^{N}$ with vertex 0 and length $\max _{S \subseteq N} v(S)$.

For a single-player game, it is obvious that there is an SSPE. Suppose an SSPE exists for each game with less than $n$ players. Then for a game with $n$ players, if any nonempty coalition $S$ leaves the game, there exists an SSPE for the subgame by the hypothesis. For each such subgame, fix one SSPE and the corresponding unique vector of expected equilibrium payoff for this subgame in $N \backslash S$, which is defined as $u^{N \backslash S}$.

Given any $(x, \alpha) \in X \times A$, assume that proposer $i$ has proposed a coalition $S$. In order to obtain the maximal payoff for $i$, we have to consider the following problem.

$$
\begin{array}{cl}
\max _{\substack{S^{\prime}: S^{\prime} \subseteq S \\
i \notin S^{\prime}}} & v(S)-\sum_{j \in S^{\prime}} \delta_{j} x_{j} \\
\text { subject to } & w_{i}+\sum_{j \in S^{\prime}} w_{j} \geq q_{S} .
\end{array}
$$

Let $\mathscr{S}(x)^{\prime}$ be the set of all maximizers of problem (6.3), and $S^{\prime}(x)$ be one of the maximizers. $S^{\prime}(x)$ can be interpreted as the set of players whose votes are bought by proposer $i$. The payoff to any $j \in S^{\prime}(x)$ is $\delta_{j} x_{j}$, and the players in $S \backslash\left(S^{\prime}(x) \cup\{i\}\right)$ are paid 0 .

Let $g_{i}(S, x)$ be the maximum value (6.3) can attain, then

$$
g_{i}(S, x)=v(S)-\sum_{j \in S^{\prime}(x)} \delta_{j} x_{j}
$$

Note that $g_{i}(S, x)$ is independent of $S^{\prime}(x) \in \mathscr{S}^{\prime}(x)$. Define a function $F\left(S^{\prime}, x\right)=$ $v(S)-\sum_{j \in S^{\prime}} \delta_{j} x_{j}$. Since $F$ is a continuous real-valued function on $2^{S \backslash\{i\}} \times X \rightarrow$ $\mathbb{R}$, and $X \rightarrow 2^{S \backslash\{i\}}$ is continunous and compact, from the maximum theorem [4], $g_{i}(S, x)$ is a continuous function of $x$.

Recall that $\alpha_{i}\left(S, z^{S}\right)$ is the probability that $i$ chooses a coalition $S$ and payoff vector $z^{S}$ over $S$. For notational simplicity, we denote $\alpha_{i}(S)$ as the probability that $i$ proposes $S$, and $\alpha_{i}\left(S, S^{\prime}(x)\right)$ as the probability that $i$ chooses $S$, and gives each $j \in S(x)^{\prime}$ a payoff $\delta_{j} x_{j}$, all $j \in S \backslash\left(S^{\prime}(x) \cup\{i\}\right)$ a payoff 0 . 
Then the value of $i$ when $j \neq i$ is the proposer is

$$
y_{i}^{j}(x, \alpha)=\sum_{S: i \notin S} \alpha_{j}(S) \delta_{i} u_{i}^{N \backslash S}+\delta_{i} x_{i} \sum_{S: i \in S} \sum_{S^{\prime}(x) \in \mathscr{S}^{\prime}(x)} \alpha_{j}\left(S, S^{\prime}(x)\right),
$$

and the value of $i$ when $i$ is the proposer is

$$
y_{i}^{i}(x, \alpha)=\sum_{\substack{S \subseteq N \\ i \in S}} \alpha_{i}(S) g_{i}(S, x) .
$$

Hence, the expected value for $i$ is

$$
y_{i}(x, \alpha)=p_{i}^{N} y_{i}^{i}(x, \alpha)+\sum_{j \in N \backslash i} p_{j}^{N} y_{i}^{j}(x, \alpha) .
$$

We can see that $y_{i}^{i}(x, \alpha)$ is a continuous function as $g_{i}(S, x)$ is continuous. Since $y_{i}^{j}(x, \alpha)$ is also continuous, we have that $y_{i}(x, \alpha)$ is continuous.

Fix any $x \in X$ and any $\alpha_{-i} \in \prod_{j \in N \backslash\{i\}} A_{j}$. Define the following function

$$
F_{i}\left(x, \alpha_{-i}\right)\left(\alpha_{i}\right)=p_{i}^{N} y_{i}^{i}\left(x, \alpha_{-i}, \alpha_{i}\right)+\sum_{j \in N \backslash\{i\}} p_{j}^{N} y_{i}^{j}\left(x, \alpha_{-i}, \alpha_{i}\right),
$$

and maximize this function with respect to $\alpha_{i} \in A_{i}$. Function (6.4) computes the expected payoff for player $i$ according to his behavior strategy to choose proposals. The maximum value of this problem and the set of maximizers are useful for constructing a correspondence from the space of pairs of strategy and payoff vector to the space itself, as the following statements show.

Let $\phi_{i}^{1}(x, \alpha)$ be the maximum value of function (6.4) and $\phi_{i}^{2}(x, \alpha)$ be the set of maximizers. Since $y_{i}(x, \alpha)$ is a continuous and real-valued function, by the maximum theory, we have that $\phi_{i}^{1}(x, \alpha)$ is continuous and $\phi_{i}^{2}(x, \alpha)$ is an upper-semicontinuous, compact correspondence.

Since $\prod_{i \in N} \phi_{i}^{1}$ maps from $X \times A \rightarrow X$ and $\prod_{i \in N} \phi_{i}^{2}$ maps from $X \times A \rightarrow A$, we get the correspondence

$$
\phi=\prod_{i \in N} \phi_{i}^{1} \times \prod_{i \in N} \phi_{i}^{2}: X \times A \rightarrow X \times A,
$$


which satisfies all the conditions of Kakutani's fixed point theorem [47] and therefore has a fixed point $\left(x^{\prime}, \alpha^{\prime}\right)$.

For any fixed point $\left(x^{\prime}, \alpha^{\prime}\right)$, we can see that the expected payoff vector $x^{\prime}$ actually corresponds to the strategy vector $\alpha^{\prime}$. We now use the fixed point $\left(x^{\prime}, \alpha^{\prime}\right)$ to show there exists an SSPE. Let us construct a strategy profile $\eta$ as follows.

(i) If the active player set is $N$, and $i$ is the proposer, he chooses his strategy according to $\alpha^{\prime}$. That is, player $i$ proposes coalition $S$ with the probability $\alpha_{i}^{\prime}(S)$, pays $\delta_{j} x_{j}^{\prime}$ to each player in $S^{\prime}(x)$, and pays 0 to each player in $S \backslash\left(S^{\prime}(x) \cup\{i\}\right)$. Player $i$ collects the remaining value of $S$. Here, $S^{\prime}(x)$ is an optimal solution of problem (6.3).

(ii) If the current player set is $N$, and $i$ is a respondent to a proposal $\left(S, z^{S}\right)$, he accepts the proposal if and only if $z_{i}^{S} \geq \delta_{i} x_{i}^{\prime}$.

(iii) If the current player set is not $N$, then players play the game with the preselected equilibrium.

We now show that strategy profile $\eta$ is an SSPE. If some player $i$ deviates from $\alpha^{\prime}$, he cannot receive a higher payoff than $x_{i}^{\prime}$, since $\alpha_{i}^{\prime}$ is a maximizer of function (6.4) and the maximum payoff he can get is $x_{i}^{\prime}=y_{i}\left(x^{\prime}, \alpha^{\prime}\right)$. This implies that the behavior strategy in (i) cannot be improved upon. From Proposition 6.1 we know that the behavior strategy (ii) is a best response. Clearly, the actions in (iii) cannot be improved. Thus, $\eta$ is an SSPE.

\subsection{Grand-coalition efficient games}

In this section, we restrict our attention to the grand-coalition efficient games, in which the only best response for any proposer is to propose the grand coalition $N$. Given any SSPE and any coalition $S$ with $i \in S$, let $M^{i}(S)$ be the 
optimal value of the following program:

$$
\begin{array}{cl}
\max _{\gamma^{i, S}(T) \in \Gamma^{i, S}} & v(S)-\sum_{j \in S \backslash i} \delta_{j} u_{j} \gamma_{j}^{i, S}(T) \\
\text { subject to } & w_{i}+\sum_{j \in S \backslash i} w_{j} \gamma_{j}^{i, S}(T) \geq q_{S} .
\end{array}
$$

Note that the above program is different from (6.1), since the proposed coalition is fixed and the above program is only maximized with respect to $\gamma^{i, S}(T)$. It is easy to see that the grand-coalition efficient games must satisfy that for any $i \in N$ and any $S \subseteq N, M^{i}(N) \geq M^{i}(S)$. In any SSPE of a grand-coalition efficient game, any proposer $i$ will always propose the grand coalition $N$ and offer $\delta_{j} u_{j}$ for each respondent $j$ that player $i$ bribes. And any respondent $j$ will accept any offer not less than $\delta_{j} u_{j}$. The class of grand-coalition efficient games is nonempty, as we will see in Section 6.5.

Interestingly, when we focus on grand-coalition efficient games, our games coincide with standard "divide the dollar" games. Such games have been widely studied in the bargaining literature starting from Baron et al. [3]. The main result of this section is that the pivotal players will extract all the gains from the grand coalition when their discount rates go to 1 .

For notational simplicity, we write $p_{i}$ instead of $p_{i}^{N}$. Given any SSPE $\eta$ and an SSPE proposal $\left(N, \gamma^{i, N}(S)\right)$, we write $\gamma^{i}(S)$ instead of $\left(N, \gamma^{i, N}(S)\right)$ and $\eta_{i}\left(\gamma^{i}(S)\right)$ instead of $\eta_{i}^{N}\left(N, \gamma^{i, N}(S)\right)$ for any proposer $i \in N$.

Given any SSPE $\eta$, let $\gamma^{i}(S)$ be an SSPE proposal and $\mathscr{F}_{i}^{N}$ be the set of all SSPE proposals of player $i$ with respect to $\eta$. Define the offer probabilities derived from $\eta$ as follows:

$$
\mu_{j}^{i}=\sum_{\gamma^{i}(S) \in \mathscr{F}_{i}^{N}} \gamma_{j}^{i}(S) \eta_{i}\left(\gamma^{i}(S)\right) .
$$

That is, $\mu_{j}^{i}$ is the probability that player $j$ will get the payoff $\delta_{j} u_{j}^{S}$ when $i$ is the proposer. Then, equation (6.2) can be rewritten in terms of the offer 
probabilities as

$$
u_{i}=p_{i}\left(1-\sum_{j \in N \backslash i} \delta_{j} u_{j} \mu_{j}^{i}\right)+\delta_{i} u_{i} \sum_{j \in N \backslash i} p_{j} \mu_{i}^{j} .
$$

In [82], the assumption about symmetry among players is an integral part of the study. That is, they assume that in an SSPE, players with the same type have equal expected equilibrium payoffs. In contrast, we drop this assumption in this section and develop it into a provable result. We show that players possessing the same proposer probability, discount factor, and voting weight will have the same expected equilibrium payoff. A formal definition of symmetric players is provided as follows.

Definition 6.4. Players in $S$ are called symmetric if for any $i, j \in S, p_{i}=p_{j}$, $\delta_{i}=\delta_{j}$ and $w_{i}=w_{j}$.

The next theorem shows that the symmetric players have the same expected equilibrium payoff.

Proposition 6.3. Let players in $S$ be symmetric. Then for any $i, j \in S, u_{i}=u_{j}$ in any SSPE of any grand-coalition efficient game.

Proof. Let $p_{i}=p, \delta_{i}=\delta$ and $w_{i}=w$ for all $i \in S$. Given any SSPE $\eta$, let $\gamma^{i}(S)$ be an SSPE proposal of proposer $i$ with respect to $\eta$, and $T_{i}=$ $\left\{k \in N \mid \gamma^{i}(S)=1\right\} \cup\{i\}$. Define a function $\theta\left(T_{i}\right)=1-\sum_{k \in T_{i}} \delta_{k} u_{k}$. Then, from equation (6.5) we have that

$$
u_{i}=p \theta\left(T_{i}\right)+p \delta u_{i}+\delta u_{i} \sum_{k \in N \backslash\{i\}} p_{k} \mu_{i}^{k} .
$$

Equation (6.6) implies that

$$
u_{i}=\frac{p \theta\left(T_{i}\right)}{1-\delta\left(\sum_{k \in N \backslash\{i\}} p_{k} \mu_{i}^{k}+p\right)} .
$$


Suppose that there are two symmetric players $i, j \in S$ with different expected equilibrium payoffs, i.e., $u_{i} \neq u_{j}$. Without loss of generality, we assume that $u_{i}>u_{j}$. We first show that

$$
\theta\left(T_{j}\right)>\theta\left(T_{i}\right)
$$

Note that for any $T \subseteq N$ with $i \in T$ and $\sum_{i \in T} w_{i} \geq q_{N}$ we must have $\theta\left(T_{i}\right) \geq$ $\theta(T)$. Therefore, if $j \in T_{i}$, inequality (6.8) is obviously true. If $j \notin T_{i}$, then

$\theta\left(T_{j}\right) \geq \theta\left(T_{i} \cup\{j\} \backslash\{i\}\right)=1-\sum_{k \in T_{i}} \delta_{k} u_{k}-\delta u_{j}+\delta u_{i}=\theta\left(T_{i}\right)-\delta u_{j}+\delta u_{i}>\theta\left(T_{i}\right)$.

Now we show that $\mu_{i}^{k} \leq \mu_{j}^{k}$ for any $k \in N \backslash\{i, j\}$ and $\mu_{i}^{j} \leq \mu_{j}^{i}$. Note that players $i$ and $j$ have the same number of votes, i.e., $w_{i}=w_{j}$. However, $\delta u_{i}>$ $\delta u_{j}$. This can be interpreted as the average price of the votes of $i$ is heigher than that from $j$. Therefore, the votes of $j$ are more likely to be bought by player $k$. Thus we have $\mu_{i}^{k} \leq \mu_{j}^{k}$. To show $\mu_{i}^{j} \leq \mu_{j}^{i}$, it suffices to show that $\mu_{j}^{i} \neq 1$ implies $\mu_{i}^{j}=0$. Suppose this is not the case. Then in other words, there is some $T_{i}$ with $j \notin T_{i}$ and there is some $T_{j}$ with $i \in T_{j}$. Since $i \in T_{j}$, we have $\theta\left(T_{i}\right) \geq \theta\left(T_{j}\right)$. However, we have already seen that $\theta\left(T_{j}\right)>\theta\left(T_{i}\right)$. A contradiction.

By the above argument and equation (6.7), we can conclude that $u_{i}<u_{j}$, which contradicts the hypothesis.

Our next result shows that for any pivotal player $i$ with the proposer probability $p_{i} \geq \frac{1}{n}$, if his discount rate is at least the discount rates of other symmetric players, he will have a higher expected equilibrium payoff. On the other hand, for any dispensable player with the proposer probability not greater than $\frac{1}{n}$, his expected equilibrium payoff will be strictly less than that of symmetric players.

Proposition 6.4. Given any grand-coalition efficient game, let players in $N \backslash\{k\}$ be symmetric.

(i) If $k$ is a pivotal player with $p_{k} \geq \frac{1}{n}$ and $\delta_{k} \geq \delta_{i}$ for all $i \in N \backslash\{k\}$, then $u_{k} \geq u_{i}$ for all $i \in N \backslash\{k\}$. 
(ii) If $k$ is a dispensable player with $p_{k} \leq \frac{1}{n}$, then $u_{k}<u_{i}$ for all $i \in N \backslash\{k\}$. Proof. Since the players in $N \backslash\{k\}$ are symmetric, we can set $p_{i}=p, \delta_{i}=\delta$, $w_{i}=1$ for all $i \in N \backslash\{k\}$. By Proposition 6.3, we must have $u_{i}=u$ for all $i \in N \backslash\{k\}$.

(i) Since $k$ is a pivotal player, it follows from (6.5) that

$$
u_{k}=p_{k}\left[1-\left(q_{N}-w_{k}\right) \delta u\right]+\left(1-p_{k}\right) \delta_{k} u_{k}
$$

and for all $i \in N \backslash\{k\}$

$$
u=p\left[1-\delta_{k} u_{k}-\left(q_{N}-w_{k}-1\right) \delta u\right]+p_{k} \mu_{i}^{k} \delta u+\sum_{j \in N \backslash\{k, i\}} p \mu_{i}^{j} \delta u .
$$

Note that $\sum_{j \in N \backslash\{k\}} \mu_{j}^{k}=q_{N}-w_{k}$ and $\sum_{j \in N \backslash\{k, i\}} \mu_{j}^{i}=q_{N}-w_{k}-1$ for each $i \in$ $N \backslash\{k\}$. Moreover, it holds that

$$
p=\frac{1-p_{k}}{n-1} .
$$

For all $i \in N \backslash\{k\}$, sum over all equations (6.10), we have

$$
\begin{aligned}
(n-1) u= & p(n-1)\left[1-\delta_{k} u_{k}-\left(q_{N}-w_{k}-1\right) \delta u\right] \\
& +p_{k}\left(q_{N}-w_{k}\right) \delta u+p(n-1)\left(q_{N}-w_{k}-1\right) \delta u \\
= & p(n-1)\left(1-\delta_{k} u_{k}\right)+p_{k}\left(q_{N}-w_{k}\right) \delta u \\
= & \left(1-p_{k}\right)\left(1-\delta_{k} u_{k}\right)+p_{k}\left(q_{N}-w_{k}\right) \delta u \\
= & 1-u_{k},
\end{aligned}
$$

where the third equality follows from (6.11), and the last equality follows from (6.9). Thus, we have

$$
u_{k}+(n-1) u=1 .
$$

By (6.9) and (6.12), we have

$$
u=\frac{\left(1-\delta_{k}\right)\left(1-p_{k}\right)}{(n-1)\left(1-\delta_{k}\right)+p_{k}\left[(n-1) \delta_{k}-\left(q_{N}-w_{k}\right) \delta\right]},
$$


and

$$
u_{k}=\frac{p_{k}\left[(n-1)-\delta\left(q_{N}-w_{k}\right)\right]}{(n-1)\left(1-\delta_{k}\right)+p_{k}\left[(n-1) \delta_{k}-\left(q_{N}-w_{k}\right) \delta\right]} .
$$

Note that $q_{N}-w_{k} \leq n-1$ and $\delta \leq \delta_{k}$, we must have

$$
(n-1)\left(1-\delta_{k}\right)+p_{k}\left[(n-1) \delta_{k}-\left(q_{N}-w_{k}\right) \delta\right] \geq 0 .
$$

Let $f_{1}\left(p_{k}\right)=\left(1-\delta_{k}\right)\left(p_{k}-1\right)$ and $f_{2}\left(p_{k}\right)=p_{k}\left[(n-1)-\delta\left(q_{N}-w_{k}\right)\right]$. To prove the proposition, it suffices to show that for any $p_{k} \geq \frac{1}{n}$ and $\delta_{k} \geq \delta$, $f_{1}\left(p_{k}\right) \leq f_{2}\left(p_{k}\right)$. We first show that $f_{1}\left(\frac{1}{n}\right) \leq f_{2}\left(\frac{1}{n}\right)$.

$$
f_{1}\left(\frac{1}{n}\right)=\frac{(n-1)-\delta_{k}(n-1)}{n} \leq \frac{(n-1)-\delta\left(q_{N}-w_{k}\right)}{n}=f_{2}\left(\frac{1}{n}\right),
$$

where the inequality follows from the fact that $q_{N}-w_{k} \leq n-1$ and $\delta \leq$ $\delta_{k}$. We next show that $f_{1}^{\prime}\left(q_{k}\right)<0$ and $f_{2}^{\prime}\left(q_{k}\right)>0$. In fact, it is easy to see that $f_{1}^{\prime}\left(q_{k}\right)=-\left(1-\delta_{k}\right)<0$ and $f_{2}^{\prime}\left(q_{k}\right)=(n-1)-\delta\left(q_{N}-w_{k}\right)>0$, which implies the desired result.

(ii) Since $k$ is a dispensable player, it follows from (6.5) and Proposition 6.3 that

$$
u_{k}=p_{k}\left[1-\left(q_{N}-w_{k}\right) \delta u\right]
$$

and for all $i \in N \backslash\{k\}$

$$
u=p\left[1-\left(q_{N}-1\right) \delta u\right]+p_{k} \mu_{i}^{k} \delta u+\sum_{j \in N \backslash\{k, i\}} p \mu_{i}^{j} \delta u .
$$

Note that $\sum_{j \in N \backslash\{k\}} \mu_{j}^{k}=q_{N}-w_{k}$ and for each $i \in N \backslash\{k\}, \sum_{j \in N \backslash\{k, i\}} \mu_{j}^{i}=$ $q_{N}-1$. For all $i \in N \backslash\{k\}$, sum equations (6.14), we have

$$
\begin{aligned}
(n-1) u= & p(n-1)\left[1-\left(q_{N}-1\right) \delta u\right] \\
& +p_{k}\left(q_{N}-w_{k}\right) \delta u+p(n-1)\left(q_{N}-1\right) \delta u \\
= & p(n-1)+p_{k}\left(q_{N}-w_{k}\right) \delta u \\
= & 1-p_{k}+p_{k}\left(q_{N}-w_{k}\right) \delta u \\
= & 1-u_{k},
\end{aligned}
$$


where the third equality follows from (6.11), and the last equality follows from (6.13). Thus, we have

$$
u_{k}+(n-1) u=1
$$

Then (6.12) and (6.17) imply that

$$
u_{k}=\frac{p_{k}\left[(n-1)-\delta\left(q_{N}-w_{k}\right)\right]}{(n-1)-p_{k} \delta\left(q_{N}-w_{k}\right)},
$$

and

$$
u=\frac{1-p_{k}}{(n-1)-p_{k} \delta\left(q_{N}-w_{k}\right)} .
$$

Note that $(n-1)-p_{k} \delta\left(q_{N}-w_{k}\right)>0$, it suffices to show that

$$
p_{k}\left[(n-1)-\delta\left(q_{N}-w_{k}\right)\right]<1-p_{k}
$$

for any $p_{k} \leq \frac{1}{n}$. Let $g_{1}\left(p_{k}\right)=p_{k}\left[(n-1)-\delta\left(q_{N}-w_{k}\right)\right]$ and $g_{2}\left(p_{k}\right)=1-p_{k}$. We first show that $g_{1}\left(\frac{1}{n}\right)<g_{2}\left(\frac{1}{n}\right)$.

$$
g_{1}\left(\frac{1}{n}\right)=\frac{n-1-\delta\left(q_{N}-w_{k}\right)}{n}<\frac{n-1}{n}=g_{2}\left(\frac{1}{n}\right),
$$

where the inequality follows from the fact that $n-1 \geq q_{N}-w_{k}$ and $\delta \in(0,1)$. Note that $g_{1}^{\prime}\left(p_{k}\right)=(n-1)-\delta\left(q_{N}-w_{k}\right)>0$ and $g_{2}^{\prime}\left(p_{k}\right)=-1<0$. Therefore, we must have $g_{1}\left(p_{k}\right)<g_{2}\left(p_{k}\right)$ for any $p_{k} \leq \frac{1}{n}$.

Note that in equation (6.12), when $\delta_{k}$ goes to 1 , the pivotal player extracts all the gains of the grand coalition. This makes us wonder whether it is a general result if the pivotal players are more than just one. The following result shows that this conjecture is true. That is, the pivotal players extract all the value of the grand coalition when their discount factors are close to 1 .

Proposition 6.5. Let $S$ be the set of pivotal players. If the players in $N \backslash S$ are symmetric, then $\sum_{k \in S} u_{k}=1$ as $\delta_{k}$ goes to 1 for all $k \in S$ in any grandcoalition efficient game. 
Proof. Since the players in $N \backslash S$ are symmetric, we can set $p_{i}=p, \delta_{i}=\delta$ and $w_{i}=1$ for each $i \in N \backslash S$. By Proposition 6.3, we must have $u_{i}=u$ for any $i \in N \backslash S$. Define $p(S)=\sum_{k \in S} p_{k}, w(S)=\sum_{k \in S} w_{k}$ and $u(S)=\sum_{k \in S} u_{k}$. Then it follows from (6.5) that for each $k \in S$

$$
u_{k}=p_{k}\left[1-\sum_{j \in S \backslash k} \delta_{j} u_{j}-\left(q_{N}-w(S)\right) \delta u\right]+\left(1-p_{k}\right) \delta_{k} u_{k} .
$$

For all $k \in S$, sum equations (6.16), we have

$$
u(S)=p(S)\left[1-\sum_{k \in S} \delta_{k} u_{k}-\left(q_{N}-w(S)\right) \delta u\right]+\sum_{k \in S} \delta_{k} u_{k} .
$$

For each $i \in N \backslash S$,

$$
\begin{aligned}
u= & p\left[1-\sum_{k \in S} \delta_{k} u_{k}-\left(q_{N}-w(S)-1\right) \delta u\right]+\sum_{k \in S} p_{k} \mu_{i}^{k} \delta u \\
& +\sum_{j \in N \backslash(S \cup\{i\})} p \mu_{i}^{j} \delta u .
\end{aligned}
$$

Note that for each $k \in S, \sum_{j \in N \backslash S} \mu_{j}^{k}=q_{N}-w(S)$ and $\sum_{j \in N \backslash(S \cup\{i\})} \mu_{j}^{i}=q_{N}-$ $w(S)-1$ for each $i \in N \backslash S$. Moreover, it holds that

$$
p(S)=1-(n-s) p
$$


For all $i \in N \backslash S$, sum all equations (6.18), we have

$$
\begin{aligned}
(n-s) u= & p(n-s)\left[1-\sum_{k \in S} \delta_{k} u_{k}-\left(q_{N}-w(S)-1\right) \delta u\right]+p(S)\left(q_{N}-w(S)\right) \delta u \\
& \quad+p(n-s)\left(q_{N}-w(S)-1\right) \delta u \\
= & p(n-s)\left(1-\sum_{k \in S} \delta_{k} u_{k}\right)+p(S)\left(q_{N}-w(S)\right) \delta u \\
= & (1-p(S))\left(1-\sum_{k \in S} \delta_{k} u_{k}\right)+p(S)\left(q_{N}-w(S)\right) \delta u \\
= & 1-u(S),
\end{aligned}
$$

where the third equality follows from (6.19), and the last equality follows from (6.17). Thus, we have

$$
(n-s) u+u(S)=1
$$

(6.16) and (6.20) yield that

$$
u_{k}=\frac{p_{k}\left[(n-s)\left(1-\sum_{j \in S \backslash k} \delta_{j} u_{j}\right)-\left(q_{N}-w(S)\right)(1-u(S \backslash\{k\})) \delta\right]}{(n-s)\left[1-\left(1-p_{k}\right) \delta_{k}\right]-\left(q_{N}-w(S)\right) p_{k} \delta} .
$$

When $\delta_{k}$ goes to 1 for each $k \in S$, we have

$$
\begin{aligned}
u_{k} & =\frac{(n-s)(1-u(S \backslash\{k\}))-\left(q_{N}-w(S)\right)(1-u(S \backslash\{k\})) \delta}{(n-s)-\left(q_{N}-w(S)\right) \delta} \\
& =1-u(S \backslash\{k\}) . \quad \square
\end{aligned}
$$

\subsection{Existence of grand-coalition efficient games}

In this section, we consider CBGMRs in 3-player situations, where each player has only one vote, and the approval of a proposal needs at least half of the approval committees' votes. We first show the existence of grand coalition efficient games by a 3-player example. Then, a necessary and sufficient condition is provided for the existence of grand coalition efficient games in $n$ 
symmetric players situations. Moreover, we show that players being symmetric is not a necessary condition for expected equilibrium payoffs being equal for all players.

The standard form of the 3-player example is as follows:

Example 6.1. Let $N=\{1,2,3\}$ be the set of players. For each player $i$, let $p_{i}^{S}=p_{i}$ for any $S \subseteq N$ be the proposer probabilities. Each player has only one vote, i.e., $w_{i}=1$ for any $i \in N$. Players have the same discount factor: $\delta_{i}=\delta$. The approval of a proposal needs at least half of the approval committees' votes.

Proposition 6.6. In Example 5.1, when the players are symmetric and $\delta$ goes to 0 , the corresponding CBGMR is a grand-coalition efficient game if and only if $\frac{1}{3}>\frac{v(\{i, j\})}{2}$ for all $i, j \in N$.

Proof. (Only if). Assume that the corresponding CBGMR is a grand-coalition efficient game, then from Proposition 6.3 we know that the expected equilibrium payoffs for all players are equal, i.e., $u_{i}=1 / 3$ for each $i \in N$. From Proposition 6.1, any respondent will only accept the payoff at least $\frac{1}{3} \delta$. Since the only best response for any proposer is to propose the grand coalition, the payoff for any proposer must be at least the payoff when he proposes any sub-coalition. Then we have

$$
1-\frac{1}{3} \delta>v(\{i, j\})
$$

When $\delta$ goes to 1 , we have $\frac{1}{3}>\frac{v(\{i, j\})}{2}$.

(If). Assume that $\frac{1}{3}>\frac{v(i, j)}{2}$, then for any $\delta$ we have $1-\frac{1}{3} \delta>v(\{i, j\})$. From Proposition 6.1 and Theorem 6.1 we know that the only best response for any proposer is to propose the grand coalition, and the expected equilibrium payoffs for players is $\left(\frac{1}{3}, \frac{1}{3}, \frac{1}{3}\right)$.

Example 6.1 is sufficient to show the existence of the grand-coalition efficient games. Besides, grand-coalition efficient games exist for $n$ symmetric players as the following proposition shows. 
Proposition 6.7. Given a CBGMR in which players are symmetric and $\delta$ goes to 0 , the CBGMR is a grand-coalition efficient game if and only if $1-\frac{q_{N}}{n}>$ $v(S)-\frac{q_{S}}{n}$ for each $S \subseteq N$.

Proposition 6.7 also provides a necessary and sufficient condition for the existence of grand-coalition efficient games when players are symmetric. Since the proof of Proposition 6.7 is in the same spirit as that of Proposition 6.6, we omit it here.

Now, let us consider a situation where the proposer probability of one player is different from those of the other two symmetric players. Let us call an SSPE in which the only best response for any proposer is to choose the grand coalition a grand-coalition efficient SSPE. In a situation like this, the following proposition concludes a property on the expected equilibrium payoff for the 3 players in any grand-coalition efficient SSPE.

Proposition 6.8. Given any grand-coalition efficient SSPE, and the corresponding expected equilibrium payoff vector $u$ for Example 6.1.

(i) If $p_{1} \geq \frac{\delta-1}{2 \delta-3}$, and player 2 and 3 are symmetric, then $u_{1} \geq u_{2}=u_{3}$.

(ii) If $p_{1} \leq \frac{1}{3-\delta}$, and player 2 and 3 are symmetric, then $u_{1} \leq u_{2}=u_{3}$.

Proof. (i) Suppose $u_{1}<u_{2}=u_{3}$, then the following equations must hold:

$$
\begin{aligned}
& u_{1}=p_{1}\left(1-\mu_{2}^{1} \delta u_{2}-\mu_{3}^{1} \delta u_{3}\right)+\left(1-p_{1}\right) \delta u_{1}, \\
& u_{2}=p_{1} \mu_{2}^{1} \delta u_{2}+p_{2}\left(1-\delta u_{1}\right), \\
& u_{3}=p_{1} \mu_{3}^{1} \delta u_{3}+p_{3}\left(1-\delta u_{1}\right) .
\end{aligned}
$$

Since player 2 and 3 are symmetric, from Proposition 6.3 we must have $u_{2}=$ $u_{3}$. Since $p_{2}=p_{3}$ and $\mu_{2}^{1}+\mu_{3}^{1}=1$, it is easy to see from the above equations that $\mu_{2}^{1}=\mu_{3}^{1}=\frac{1}{2}$. Thus, we calculate that

$$
\begin{aligned}
u_{1} & =\frac{1-p_{1}-\delta+p_{1} \delta}{2-2 \delta+p_{1} \delta}, \\
u_{2}=u_{3} & =\frac{2 p_{1}-p_{1} \delta}{2-2 \delta+p_{1} \delta} .
\end{aligned}
$$


Since $u_{1}<u_{2}=u_{3}$, we have $p_{1}<\frac{\delta-1}{2 \delta-3}$. However, $p_{1} \geq \frac{\delta-1}{2 \delta-3}$, a contradiction.

(ii) Suppose $u_{1}>u_{2}=u_{3}$, then the following equations must hold:

$$
\begin{aligned}
& u_{1}=p_{1}\left(1-\mu_{2}^{1} \delta u_{2}-\mu_{3}^{1} \delta u_{3}\right), \\
& u_{2}=p_{1} \mu_{2}^{1} \delta u_{2}+p_{2}\left(1-\delta u_{3}\right)+p_{3} \delta u_{2}, \\
& u_{3}=p_{1} \mu_{3}^{1} \delta u_{3}+p_{2} \delta u_{3}+p_{3}\left(1-\delta u_{2}\right) .
\end{aligned}
$$

As $u_{2}=u_{3}, p_{2}=p_{3}$ and $\mu_{2}^{1}+\mu_{3}^{1}=1$, we have $\mu_{2}^{1}=\mu_{3}^{1}=\frac{1}{2}$. From equations (6.22), we have

$$
\begin{aligned}
u_{1} & =\frac{2 p_{1}-\delta p_{1}}{2-p_{1} \delta}, \\
u_{2}=u_{3} & =\frac{1-p_{1}}{2-p_{1} \delta} .
\end{aligned}
$$

Since $u_{1}>u_{2}=u_{3}, p_{1}>\frac{1}{3-\delta}$. However, $p_{1} \leq \frac{1}{3-\delta}$, a contradiction.

In Proposition 6.8, one may notice that even if $p_{1}$ is different from the proposer probability of the symmetric players, the expected equilibrium payoffs for the 3 players can still be equal. This fact can be seen from the following example.

Example 6.2. In Example 6.1, let players 2,3 be symmetric. Set $p_{1}=\frac{1}{3-\delta}$, and $p_{2}=p_{3}$. Construct a strategy profile $\eta$ as follows.

(i) If player 1 is the proposer, he always proposes the grand coalition and selects player 2 or 3 with equal probability to pay $\frac{1}{3} \delta$. The other player is paid 0 .

(ii) If player 2 is the proposer, he always proposes the grand coalition and selects player 3 to pay $\frac{1}{3} \delta$. Player 1 is paid 0 .

(iii) If player 3 is the proposer, he always proposes the grand coalition and selects player 2 to pay $\frac{1}{3} \delta$. Player 1 is paid 0 .

(iv) For any player $i \in N$, if player $i$ is a respondent, he only accepts payoff no less than $\frac{1}{3} \delta$.

It is easy to see $\eta$ is a grand-coalition efficient SSPE, and $u_{1}=u_{2}=u_{3}=\frac{1}{3}$. 
The above example also indicates that the symmetry of players is not a necessary condition on the equality of expected equilibrium payoffs in CBGMRs. 


\section{Chapter 7}

\section{Nash bargaining with rational threats in generalized partition function games}

This chapter proposes a class of games, called general partition function games (GPFG), in which internalities and externalities across coalitions are both considered. In order to obtain a reasonable worth for each coalition in these games, the Nash bargaining model is employed and we show that there exists at most one rational Nash bargaining payoff (RNBP) for any deviating coalition. We provide two sufficient conditions for the existences of rational threat partition pairs (RTPPs), which are essential for the determination of RNBP. Moreover, by viewing the RNBP as the worth of a deviating coalition, we define the core of the corresponding characteristic function game. We further provide two sufficient conditions for the non-emptiness of the core.

\subsection{Introduction}

One of the most important problems in (transferable utility) cooperative game theory is how to allocate the total surplus of the grand coalition fairly and properly to the players. Various solution notions have been put forward for 
solving this problem, like core, nucleolus, bargaining set, and Shapley value. The common feature of all these solution concepts is that their definitions mainly rely on the worths of coalitions, which can be deemed as the bargaining power of coalitions in the determination of the allocation. In a CFF games, it is intrinsically assumed that what a coalition can achieve has nothing to do with the outside players, so the worth of each coalition is confirmed and clear.

However, plenty of economic scenarios involve externalities across coalitions, which means that the worth of a coalition depends not only on the coalition in question, but also on the economic behavior of external players. In such a situation, each coalition should ask itself, "if we decide to depart from the grand union, how will the remaining players react?" and "how much will we get if the deviation happens?". This has raised a series of researches starting from Thrall and Lucas [83], who introduced a new cooperative game concept called the partition function form game used for modeling the settings with externalities. Since then, the research in this area has attracted widespread interest; see, for example, [7], [8], [10], [13], [24], [25], [36], [41], [56], [63], [73], and [74]. To see a simple example where externalities exist, one can imagine the situation that in a sales market, the competitive pressure from other alliances of sellers will indeed make a bad influence on the income of a formed coalition. More applications of PFF games in economic situations can be found in [92].

Apparently, the scope of application of the PFF game is broader than that of the CFF game, but it has the same feature as the latter one: when a coalition $S$ deviates from the grand coalition $N$, whether or not the partition of $N \backslash S$ affects the worth of $S$ does not change the fact that we need not consider the internal coalition structure of $S$. This immediately raises the questions of what if we look inside of the coalition in question one step further, and what if the internal coalition structure of a deviating coalition also affects the profits of external players. We believe that depth studies of these issues can be significant in both theory and practical applications. For instance, the profit of a company mostly requires a reasonable and efficient organizational structure. The more efficient the organizational structure is, the more benefit the company will get, so the more competitive this company will be in the market. However, the efficient organization of a company can 
not be invariable because it should also be matched with the current market environment. When the external environment changes, the organizational structure of the company must also adjust accordingly. This example reflects the situation in which both internalities and externalities occur at the same time, and also reveals the importance and necessity of considering the internal structures of coalitions.

The main aim of this chapter is to investigate the cooperative games where the worth of each coalition depends on the partition of itself as well as the coalition structure of the nonmembers. We restrict our attention to the case where there are only two coalitions in the game; i.e., the remaining players will stick to the original binding agreement and form a single coalition if some other coalition leaves. Clearly, the partitions of any coalition and its complement produce a great impact on their final payoffs. For a better description of this phenomenon, we define a class of games called general partition function games (GPFGs), which is a formulation that assigns a real numbered outcome to each coalition associated with each partition of the coalition itself and each partition of its complement. We can, of course, improve our model by further generalizing it to the case where there are more than two coalitions, but that will make the analysis and the determination of the worth of each coalition more complex. The difficulties in this kind of generalization are that, first, which coalitions will form after the departure of a coalition; and second, which partition will each coalition choose. This part of work is beyond the scope of this chapter and will be discussed in future research.

Once the games are defined, the next question naturally arises: how do we evaluate the worth of a specific coalition when internalities and externalities coexist? This problem seems more difficult than the one where only externalities are presented. Ideally, a coalition can maximize its own payoff with complete information or perfect expectation of the coalition structure of its complement. In PFF games, many predefined expectation rules on the partition of external players have been proposed in the literature. For example, the $\alpha$-core and $\beta$-core provided by Aumann and Peleg [2] both include the idea that each deviating coalition pessimistically conjectures that the outside players will choose the strategy which minimizes the payoff of deviating players. On the contrary, Shenoy [79] argued that the deviating players are 
very optimistic and expect that external players will act in such a way that the deviating coalition's payoff is maximized. Other discussions like the external players partitioning themselves into singletons [24, 36] or forming a complement [56]. A full discussion about the formation of external players can be found in [8], where the axiomatic characterizations on different kinds of expectation formation rules are also provided. The main advantage of the predetermined rule on the partition of external players is that any PFF game will degenerate to a CFF game, which makes the analysis much more simplified.

However, we argue that it is not convincing to presume a fixed form of behavior for the outside players and ignore the strategy choices of any complementary coalition. The same argument can also be found in [10], who used a noncooperative approach in which players of the two coalitions sequentially choose the strategy which maximizes their own worth and hurts the other as much as possible. While the strategic perspective taken by Borm et al. [10] is illuminating, there is still a shortcoming: in most cases, the final payoffs for both of the two coalitions are not optimal. In the current chapter, we believe that the reasonable way for both coalitions to achieve their contented outcomes is that they can bargain with one another and agree on a rational joint plan of action. This idea hints at the potential possibility of collaboration between coalitions, which is very different from the non-cooperative approach taken by Borm et al. [10].

In this study, we apply the well known two-person bargaining model, which is initially proposed by Nash $[65,66]$, to have better insights into the players' actions and assess the worth of a coalition. The bargaining model in this chapter can be simply described as follows: first, $S$ and $N \backslash S$ announce the partitions of themselves simultaneously. The partition chosen by one coalition can be seen as a threat to another: if one coalition breaks the agreement of negotiation, the other coalition will implement its partition proposed in the first beginning. These threats are prepared in advance before the start of the bargaining process to settle the disagreement point, which is a pair of payoffs for the two coalitions when the threats are carried out. Since the Nash bargaining solution, which satisfies a list of axioms given by Nash [65] 
in defining fairness, is largely dependent on the disagreement point, the payoff from the cooperative agreement for any coalition is uniquely determined by the threats which were chosen in the first step. Then, by realizing this point, any coalition will have the incentive to act more rationally and choose the most preferable partition so as to maximize its final gain. This pair of rational actions, which will be formally defined in Section 7.3, is called rational threat partition pair (RTPP) and the corresponding Nash bargaining solution is called rational Nash bargaining payoff (RNBP). The main goal of our study is to find the RNBP for any coalition as a measurement of its own worth and we only have to restrict our attention to the exploration of RTPPs. We first show that despite the multiplicity of the RTPP, the RNBP is unique if RTPPs exist. Then two sufficient conditions for the existences of RTPPs are provided. We further examine the exact forms of threats when the GPFGs have positive or negative internalities and positive or negative externalities. Moreover, we restrict our attention to the GPFGs where RTPPs do exist, and any coalition in this situation can have a Nash bargaining payoff as the evaluation of its own worth. The core of the associated games in characteristic function form is defined and by extending the convexity of PFF games defined in [36], we provide two sufficient conditions for the non-emptiness of the core.

The chapter is organized as follows: Section 7.2 provides new games in more a generalized partition function form. In Section 7.3, a bargaining model between two complemented coalitions is introduced and we show that there is at most one RNBP for any coalition. Section 7.4 contains two sufficient conditions for the existence of RTPPs. We discuss the non-emptiness of the core derived from RNBP in Section 7.5. Section 7.6 concludes this chapter with some further remarks.

\subsection{The general and extended partition function games}

Let $N=\{1, \ldots, n\}$ be a set of players. Recall that a partition of $S \subseteq N$ is defined by $\rho=\left\{S_{1}, \ldots, S_{k}\right\}$, where $1 \leq k \leq|S|, S_{i} \neq \emptyset$ for $i=1, \ldots, k, S_{i} \cap S_{j}=\emptyset$ for $i, j=1, \ldots, k(i \neq j)$, and $\bigcup_{i=1}^{k} S_{i}=S$. By $\Pi_{S}$ we denote the set of all partitions of $S$. Elements of a partition is called atoms. A partition $\rho$ of $S$ is 
finer than another partition $\rho^{\prime}$ if for any $T \in \rho, T \subseteq T^{\prime}$ for some $T^{\prime} \in \rho^{\prime}$. Equivalently, $\rho^{\prime}$ is coarser than $\rho$.

Although PFF games are more appropriate than CFF games for dealing with the situations where externalities exist, there are still limitations. To see this, consider the following example.

Example 7.1. Four Cournot oligopolists produce output at no cost in a homogeneous market with a linear demand curve: $p=A-b x$, where $p$ is the price of the production, $b$ a constant parameter, and $x$ the total output in the market. Firms are free to form coalitions among themselves. If any coalition $S$ is formed, it has to choose a partition $\rho$ for itself so as to decide its own output quantity $q_{\rho}$, which is uniquely determined by its partition. To paraphrase: different organization structure leads to different production efficiency.

Let $N=\{1,2,3,4\}$ be the set of firms, $A=5$ and $b=1$. Consider the situation where firms 1 and 2 are deciding to form a cartel, and then firms 3 and 4 form another. In this case, there are two different types of output quantity, depend on the partitions, for each cartel to choose from. Assume that $q_{\{\{1\},\{2\}\}}=$ $q_{\{\{3,4\}\}}=2$ and $q_{\{\{1,2\}\}}=q_{\{\{3\},\{4\}\}}=1$. Then the profits for the two coalitions with respect to their partitions can be easily calculated and shown in Figure 7.1.

\begin{tabular}{r|c|c|}
\multicolumn{1}{c}{} & $\{3\},\{4\}$ & $\{3,4\}$ \\
\cline { 2 - 3 }$\{1\},\{2\}$ & 4,2 & 2,2 \\
\cline { 2 - 3 }$\{1,2\}$ & 3,3 & 2,4 \\
\cline { 2 - 3 } & &
\end{tabular}

FIGURE 7.1: The profits for coalitions $\{1,2\}$ and $\{3,4\}$.

Obviously, this example can not be described by any CFF game or PFF game. The basic reason is that the impact factors of the profit of a coalition not only come from the behavior of outside players, but also its own strategy on partitions. One may notice that for any duopoly, the normal form representation is adequate for the description of this game, as we did in Figure 7.1. But this approach is appropriate for only the specific situation where the two coalitions in question have already been fixed. If we want to represent this game in a more general way, the normal form illustration is still limited and we need to find another suitable method. 
In the following, we will introduce a new cooperative game concept applying to the situations like that described in Example 7.1. Before that, through this chapter, we make the following assumption for the sake of simplicity of analysis.

\section{Assumption 1.}

(i) The internal partition of a coalition is only determined by the coalition itself.

(ii) The decision of a coalition for its internal partition is a kind of private information.

(iii) When a coalition $S$ deviates, the rest of players form coalition $N \backslash S$.

Assumption (i) expresses that every coalition has the right to decide the partition for itself. Assumption (ii) is a natural consequence of the definition of GPFGs. To reject assumption (ii) is to assume that any coalition $S$ can make the optimal decision for itself since it has already been informed of the partition of external players, which will certainly shrink a GPFG to a CFF game and make our generalization insignificant. The deviation assumption (iii) restricts the scenario that the cooperative behavior of some players will drive the nonmembers to form another coalition, so there will be only two coalitions under consideration if any event of defection happens. We can surely remove assumption (iii) for considering a more general situation where there are more than two coalitions formed after the deviation, but that will take the complexity of the research to another level. This kind of generalization is beyond the scope of this chapter, and we shall leave it for future work.

For notation simplicity, in the following of the chapter, we denote by $[S]$ the partition of $S$ to singletons and write $\underline{S}$ instead of $N \backslash S$ for any $S \subseteq N$. Given any coalition $S \subseteq N$, any $\rho \in \Pi_{S}$ and any $\bar{\rho} \in \Pi_{S}$, we denote by $\rho \mid \bar{\rho}$ a partition pair with respect to $S$ and by $(S ; \rho \mid \rho)$ a general embedded coalition. The set of all general embedded coalitions is denoted by $G E C(N)$.

Definition 7.1. A general partition function game $(G P F G)$ is a pair $(N, v)$ where $N$ is the set of players and $v: G E C(N) \rightarrow \mathbb{R}$ is a map that assigns 
to each general embedded coalition a real value and with the property that $v\left(\emptyset ; \emptyset \mid \rho_{N}\right)=0$ for every $\rho_{N} \in \Pi_{N}$.

For any GPFG, $v(S ; \rho \mid \rho)$ represents the worth of $S$ under the condition that $S$ chooses the partition $\rho$, and $\underline{S}$ chooses the partition $\rho$. Apparently, this representation method is more appropriate for the situation in Example 7.1. For instance, if $\{1,2\}$ is the deviating coalition, the worths of $\{1,2\}$ with respect to different partition pairs can be written by

$$
\begin{gathered}
v(\{1,2\} ;\{\{1,2\}\} \mid\{\{3,4\}\})=v(\{1,2\} ;\{\{1\},\{2\}\} \mid\{\{3,4\}\})=2, \\
v(\{1,2\} ;\{\{1,2\}\} \mid\{\{3\},\{4\}\})=3, v(\{1,2\} ;\{\{1\},\{2\}\} \mid\{\{3\},\{4\}\})=4 .
\end{gathered}
$$

Definition 7.2. A GPFG is said to have positive (negative) internalities if for any general embedded coalition $(S ; \rho \mid \underline{\rho})$ and $\left(S ; \rho^{\prime} \mid \underline{\rho}\right)$ such that $\rho$ is coarser (finer) than $\rho^{\prime}$,

$$
v(S ; \rho \mid \underline{\rho}) \geq v\left(S ; \rho^{\prime} \mid \underline{\rho}\right) .
$$

Definition 7.3. A GPFG is said to have positive (negative) externalities if for any general embedded coalition $(S ; \rho \mid \underline{\rho})$ and $\left(S ; \rho \mid \underline{\rho}^{\prime}\right)$ such that $\underline{\rho}$ is coarser (finer) than $\underline{\rho}^{\prime}$,

$$
v(S ; \rho \mid \underline{\rho}) \geq v\left(S ; \rho \mid \underline{\rho}^{\prime}\right) .
$$

A GPFG has positive (negative) internalities meaning that the merge of atoms in the coalition $S$ does not make $S$ worse (better) off. A GPFG has positive (negative) externalities meaning that the merge of atoms in the outside coalition $\underline{S}$ does not make $S$ worse (better) off.

Many questions will arise from the games defined above. The main problem is how should a deviating coalition evaluate its own worth under the circumstance that externalities and internalities coexist? Or in other words, what kind of partition will a coalition choose when it separates away from the grand coalition? These problems can be even more complex if we consider the situation where coalitions may randomize their choices on the partitions. It is, indeed, possible that the behavior of any coalition is not deterministic but regulated by some probability rule. To consider this kind of situation in the model, we further extend our class of GPFGs to a broader class of games. 
A mixed partition $\hat{\rho}$ of coalition $S$ is a probability distribution on $\Pi_{S}$. The set of all mixed partitions for the coalition $S$ is given by $P(S)$. Given any coalition $S \subseteq N$, any $\hat{\rho} \in P(S)$ and any $\underline{\hat{\rho}} \in P(\underline{S})$, we denote by $\hat{\rho} \mid \underline{\hat{\rho}}$ a mixed partition pair and by $(S ; \hat{\rho} \mid \underline{\hat{\rho}})$ a mixed embedded coalition. The set of all mixed embedded coalitions is denoted by $\operatorname{EEC}(N)$. In this chapter, we assume that the order of the two elements in a (mixed) partition pair is not significant, i.e., for any $S \subseteq N, \rho|\underline{\rho}=\underline{\rho}| \rho$ and $\hat{\rho}|\underline{\hat{\rho}}=\underline{\hat{\rho}}| \hat{\rho}$.

Definition 7.4. Let $(N, v)$ be a GPFG. The corresponding extended partition function game $(E P F G)$ is a pair $(N, u)$ where $N$ is the set of players and $u$ : $E E C(N) \rightarrow \mathbb{R}$ is a map that assigns to each $(S ; \hat{\rho} \mid \underline{\hat{\rho}}) \in E E C(N)$ the value

$$
u(S ; \hat{\rho} \mid \underline{\hat{\rho}})=\sum_{\rho \in \Pi_{S}} \sum_{\underline{\rho} \in \Pi_{\underline{S}}} \hat{\rho}(\rho) \cdot \underline{\hat{\rho}}(\underline{\rho}) \cdot v(S ; \rho \mid \underline{\rho}),
$$

where $\hat{\rho}(\rho)$ is the probability that the mixed partition $\hat{\rho}$ of coalition $S$ assigns to the partition $\rho \in \Pi_{S}$.

Note that the value $u(S ; \hat{\rho} \mid \hat{\rho})$ is the expected payoff for $S$ when the mixed partitions $\hat{\rho}$ and $\underline{\hat{\rho}}$ are both employed.

\subsection{The Nash bargaining model}

\subsubsection{The model}

The main goal of this section is to pin down a reasonable worth for a deviating coalition $S$ in the situation where internalities and externalities coexist. To accomplish this, we borrow the tool of two-person bargaining from [66]. The main idea is that $S$ and $\underline{S}$ will bargain and negotiate for working out a preferable joint plan of action so that both of them will be satisfied.

We now formally describe our bargaining model. First, for any deviating coalition $S, S$ and $\underline{S}$ have to choose their own partitions simultaneously as threats before they bargain with each other. The "threat" here means that coalition $S$ claims to choose the partition $\rho \in \Pi_{S}$ if they would not come to an agreement after the bargaining process. The same goes for the remaining 
coalition $\underline{S}: \underline{S}$ also has to choose a partition $\underline{\rho} \in \Pi_{\underline{S}}$ as a threat. We call $(\rho, \underline{\rho})$ a threat partition pair for coalition $S$.

Then the two coalitions start to negotiate with each other and the final settlement will be the solution of the optimization problem defined in the following:

Definition 7.5. Let $(N, v)$ be a GPFG, and $(N, u)$ the corresponding EPFG. A mixed partition pair $\hat{\rho}^{*} \mid \hat{\rho}^{*}$ is called the Nash bargaining partition pair for coalition $S$ under the threat partition pair $(\rho, \rho)$ if it solves the problem

$$
\begin{aligned}
\max & {[u(S ; \hat{\rho} \mid \underline{\hat{\rho}})-v(S ; \rho \mid \underline{\rho})] \cdot[u(\underline{S}, \hat{\rho} \mid \underline{\hat{\rho}})-v(\underline{S} ; \rho \mid \underline{\rho})] } \\
\text { s.t. } \quad & {[u(S ; \hat{\rho} \mid \underline{\hat{\rho}}), u(\underline{S}, \hat{\rho} \mid \underline{\hat{\rho}})] \in F(S), } \\
& u(S ; \hat{\rho} \mid \underline{\hat{\rho}}) \geq v(S ; \rho \mid \underline{\rho}), \\
& u(\underline{S}, \hat{\rho} \mid \underline{\hat{\rho}}) \geq v(\underline{S} ; \rho \mid \underline{\rho}),
\end{aligned}
$$

where

$$
F(S)=\{[u(S ; \hat{\rho} \mid \underline{\hat{\rho}}), u(\underline{S}, \hat{\rho} \mid \underline{\hat{\rho}})] \mid \hat{\rho} \in P(S), \underline{\hat{\rho}} \in P(\underline{S})\} .
$$

Here, $F(S)$ is called the feasible payoff set with respect to $S$, and the pair $[v(S ; \rho \mid \underline{\rho}), v(\underline{S} ; \rho \mid \underline{\rho})]$ the disagreement point and $\left[u\left(S ; \hat{\rho}^{*} \mid \underline{\hat{\rho}}^{*}\right), u\left(\underline{S}, \hat{\rho}^{*} \mid \underline{\hat{\rho}}^{*}\right)\right]$ the Nash bargaining payoff under the threat partition pair $(\bar{\rho}, \underline{\rho})$.

In Definition 7.5, the disagreement point represents the payoffs for the two coalitions when the negotiations broke down. Conditions " $u(S ; \hat{\rho} \mid \underline{\hat{\rho}}) \geq$ $v(S ; \rho \mid \underline{\rho}) "$ and "u($\underline{S}, \hat{\rho} \mid \underline{\hat{\rho}}) \geq v(\underline{S} ; \rho \mid \underline{\rho})$ " ensure that the final payoffs for the two coalitions when they come to an agreement are not less than the payoffs when the threats are implemented. What should be noted is that, in our model, we assume that the threat partition pair can not be randomized. This can be interpreted as due to the fact that the threat of one party is a sharp and clear declaration against the other. However, in negotiations, coalitions can choose mixed strategies on partitions, so the Nash bargaining partition pair is a mixed partition pair.

In the following, we define three kinds of boundaries of $F(S)$, the first of which is following [40]. 
- Upper-right boundary: $H_{S}=\left\{y \in F(S) \mid\right.$ there is no $z \in F(S)$ s.t. $z_{1} \geq y_{1}, z_{2} \geq$ $y_{2}$ and $\left.z \neq y\right\}$.

- Upper boundary: $U_{S}=\left\{y \in F(S) \backslash H_{S} \mid\right.$ there is no $z \in F(S)$ s.t. $\left.z_{2}>y_{2}\right\}$.

- Right boundary: $R_{S}=\left\{y \in F(S) \backslash H_{S} \mid\right.$ there is no $z \in F(S)$ s.t. $\left.z_{1}>y_{1}\right\}$.

One of the most well-known properties that a Nash bargaining solution should satisfy is Pareto optimality [48], so the next proposition needs no proof.

Proposition 7.1. For any coalition $S \subseteq N$ and any threat partition pair for $S$, the corresponding Nash bargaining payoff is in the upper-right boundary $H_{S}$.

Note that once the threat partition pair is determined, we can immediately find the corresponding Nash bargaining payoff. For notation simplicity, we denote by $[\omega(S ; \rho \mid \underline{\rho}), \omega(\underline{S} ; \rho \mid \underline{\rho})]$ the Nash bargaining payoff when the threat partition pair is $(\rho, \underline{\rho})$. A graphic description is given in Figure 7.2, where the $u_{1}$-axis and the $u_{2}$-axis represent the payoff for coalitions $S$ and $\underline{S}$ respectively.

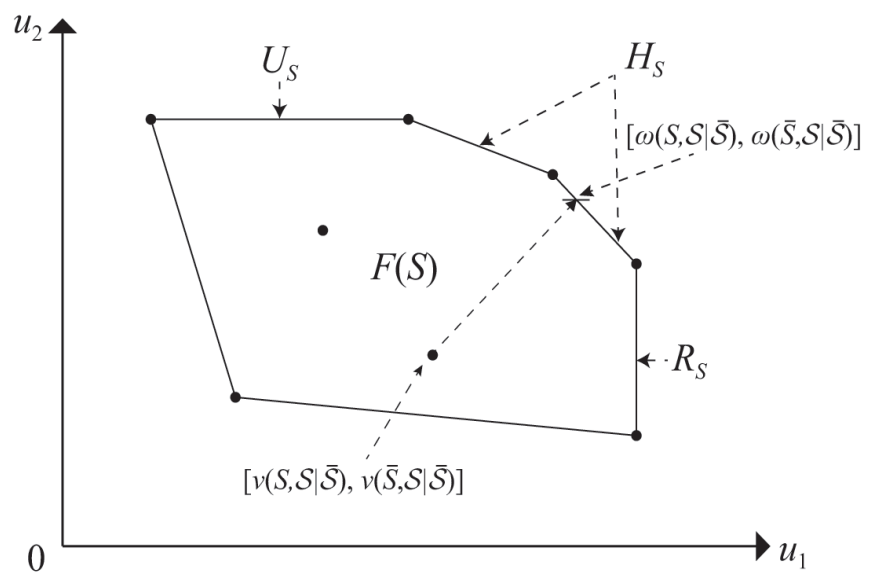

FIGURE 7.2: A graphic description of the three boundaries, and the Nash bargaining payoff when the threat partition pair is $(\rho, \underline{\rho})$.

Definition 7.6. For any coalition $S \subseteq N$, a threat partition pair $\left(\rho^{*}, \underline{\rho}^{*}\right)$ is called a rational threat partition pair (RTPP) if and only if for any $\rho \in \overline{\Pi_{S}}$ and 
any $\underline{\rho} \in \Pi_{\underline{S}}$

$$
\begin{aligned}
& \omega\left(S ; \rho^{*} \mid \underline{\rho}^{*}\right) \geq \omega\left(S ; \rho \mid \underline{\rho}^{*}\right), \\
& \omega\left(\underline{S} ; \rho^{*} \mid \underline{\rho}^{*}\right) \geq \omega\left(\underline{S} ; \rho^{*} \mid \underline{\rho}\right) .
\end{aligned}
$$

We call $\left[\omega\left(S ; \rho^{*} \mid \underline{\rho}^{*}\right), \omega\left(\underline{S} ; \rho^{*} \mid \underline{\rho}^{*}\right)\right]$ derived from $\left(\rho^{*}, \underline{\rho}^{*}\right)$ a rational Nash bargaining payoff $(R \bar{N} B P)$.

Definition 7.6 is based on the following idea. Assume that both $S$ and $\underline{S}$ can foresee the final outcome as soon as a pair of threat partitions is determined. At the outset of the negotiation, what a coalition should concern about is not the implementation of the threats but the Nash bargaining payoff engendered by the threat partition pair. Thus, given any threat of one party, the other should choose a threat partition of itself which leads to the maximization of its Nash bargaining payoff.

In this chapter, the RNBP for a coalition $S$ is considered to be the final worth of $S$. Although we can determine the coalitional worth for $S$ in many ways, the main advantage of the Nash bargaining model is that, in some sense, it improves the payoffs for both sides as much as possible. We now use Example 7.1 again for illustration. On one hand, if we assume that $\{1,2\}$ and $\{3,4\}$ choose their partitions simultaneously and independently. The first solution concept that may come to one's mind is that of Nash equilibrium. But it is hard to determine the worths of $\{1,2\}$ and $\{3,4\}$ if we use this notion, since there are three Nash equilibria in this example. If we believe that the two coalitions will rationally choose their weekly dominated strategies, which are partitions $\{\{1\},\{2\}\}$ and $\{\{3,4\}\}$, the payoff profile for the two coalitions will be $(2,2)$. On the other hand, if we use the sequential approach which is also taken by [10]; that is, if we assume that a coalition $S$ chooses the partition that is best for it on the assumption that whatever it does, the other coalition $\underline{S}$ will choose its action to hurt $S$ as much as possible, it is easy to see that the payoffs for both coalitions are 2 in this example. However, note that the unique RTPP is $(\{\{1\},\{2\}\},\{\{3,4\}\})$ and the corresponding RNBP is $(3,3)$, the payoffs for the two coalitions will be strictly improved if we employ the Nash bargaining model. 


\subsubsection{Examples}

At this stage, a question that comes naturally to mind is: does an RTPP always exist for any GPFG and any coalition $S$ ? Apparently, with no surprise, the answer is no. The first example provides a four-player GPFG situation in which the RTPP does not exist.

Example 7.2. Consider the following 4-player $\operatorname{GPFG}(N, v)$ with $N=\{1,2,3,4\}$.

$$
\begin{aligned}
& v(\{1,2\} ;\{\{1\},\{2\}\} \mid\{\{3\},\{4\}\})=v(\{3,4\} ;\{\{1\},\{2\}\} \mid\{\{3,4\}\})=0 ; \\
& v(\{1,2\} ;\{\{1\},\{2\}\} \mid\{\{3,4\}\})=v(\{3,4\} ;\{\{1\},\{2\}\} \mid\{\{3\},\{4\}\})=2 ; \\
& v(\{1,2\} ;\{\{1,2\}\} \mid\{\{3,4\}\})=v(\{3,4\} ;\{\{1,2\}\} \mid\{\{3\},\{4\}\})=2 ; \\
& v(\{1,2\} ;\{\{1,2\}\} \mid\{\{3\},\{4\}\})=v(\{3,4\} ;\{\{1,2\}\} \mid\{\{3,4\}\})=4 .
\end{aligned}
$$

If $\{1,2\}$ or $\{3,4\}$ decides to split away from $N$, there will be two partitions to choose from for each coalition. Thus there will be four possible disagreement points, which are given in Figure 7.3. The Nash bargaining payoffs derived from these disagreement points are shown in Figure 7.4. It is easy to see that there is no RTPP in this game.

\begin{tabular}{r|c|c|} 
& $\{3\},\{4\}$ & $\{3,4\}$ \\
$\{1\},\{2\}$ & 0,2 & 2,0 \\
\cline { 2 - 3 }$\{1,2\}$ & 4,2 & 2,4 \\
\cline { 2 - 3 } & &
\end{tabular}

FIGURE 7.3: The disagreement points for coalitions $\{1,2\}$ and $\{3,4\}$.

\begin{tabular}{r|c|c|} 
& $\{3\},\{4\}$ & $\{3,4\}$ \\
$\{1\},\{2\}$ & 2,4 & 4,2 \\
\cline { 2 - 3 }$\{1,2\}$ & 4,2 & 2,4 \\
\cline { 2 - 3 } & &
\end{tabular}

FIGURE 7.4: The Nash bargaining payoffs derived from the disagreement points in Figure 7.3.

Our next example illustrates that if the RTPP exists, it may not be unique. 
Example 7.3. Consider the following 5-player $\operatorname{GPFG}(N, v)$ with $N=\{1,2,3,4,5\}$. Now suppose that the coalition $\{1,2\}$ splits away from $N$ and has to bargain with $\{3,4,5\}$, then there are ten disagreement points in the bargaining process, which are given in Figure 7.5. The Nash bargaining payoffs derived from these disagreement points can be easily calculated and shown in Figure 7.6. We can clearly see that there are four RTPPs, which are $(\{\{1\},\{2\}\},\{\{3\},\{4\},\{5\}\})$, $(\{\{1,2\}\},\{\{3\},\{4\},\{5\}\}),(\{\{1\},\{2\}\},\{\{3,4,5\}\}),(\{\{1,2\}\},\{\{3,4,5\}\})$.

\begin{tabular}{r|c|c|c|c|c|} 
& $\{3\},\{4\},\{5\}$ & $\{3,4,5\}$ & $\{3,4\},\{5\}$ & $\{3,5\},\{4\}$ & $\{3\},\{4,5\}$ \\
\cline { 2 - 6 }$\{1\},\{2\}$ & 2,2 & 2,1 & 3,3 & $2.5,1$ & 2,0 \\
\cline { 2 - 6 }$\{1,2\}$ & 1,2 & 1,1 & 3,1 & 4,1 & 3,0 \\
\cline { 2 - 6 } & & &
\end{tabular}

FIGURE 7.5: The disagreement points for coalitions $\{1,2\}$ and $\{3,4,5\}$.

\begin{tabular}{r|c|c|c|c|c|} 
& $\{3\},\{4\},\{5\}$ & $\{3,4,5\}$ & $\{3,4\},\{5\}$ & $\{3,5\},\{4\}$ & $\{3\},\{4,5\}$ \\
\cline { 2 - 6 }$\{1\},\{2\}$ & 3,3 & 3,3 & 3,3 & $3.25,2.5$ & $3.25,2.5$ \\
\cline { 2 - 6 }$\{1,2\}$ & 3,3 & 3,3 & $3.5,2$ & 4,1 & $3.75,1.5$ \\
\cline { 2 - 6 } & & &
\end{tabular}

FIGURE 7.6: The Nash bargaining payoffs derived from the disagreement points in Figure 7.5.

Observe that in Example 7.3, despite the non-uniqueness of the RTPPs, the RNBPs are the same. This phenomenon makes us wonder if this result is a general feature of the problem which holds regardless of the game under consideration. This is what we turn to next.

\subsubsection{Uniqueness of RNBP}

Theorem 7.1. For any $\operatorname{GPFG}(N, v)$ and any coalition $S$, there is at most one $R N B P$ for $S$ and $\underline{S}$.

To show the validity of Theorem 7.1, the following lemma is taken into account.

Lemma 7.2. Let $(N, v)$ be a GPFG and $(N, u)$ the corresponding EPFG. Given any coalition $S \subseteq N$, for any $R T P P\left(\rho^{*}, \underline{\rho}^{*}\right)$, any $\rho \in \Pi_{S}$ and any $\underline{\rho} \in \Pi_{\underline{S}}$ we 
have

$$
\begin{aligned}
& \omega\left(S ; \rho^{*} \mid \underline{\rho}^{*}\right) \leq \omega\left(S ; \rho^{*} \mid \underline{\rho}\right), \\
& \omega\left(\underline{S} ; \rho^{*} \mid \underline{\rho}^{*}\right) \leq \omega\left(\underline{S} ; \rho \mid \underline{\rho}^{*}\right) .
\end{aligned}
$$

Proof. We first show that for any $\underline{\rho} \in \Pi_{\underline{S}}, \omega\left(S ; \rho^{*} \mid \underline{\rho}^{*}\right) \leq \omega\left(S ; \rho^{*} \mid \rho\right)$. Suppose that there is $\underline{\rho} \in \Pi_{\underline{S}}$ such that

$$
\omega\left(S ; \rho^{*} \mid \underline{\rho}^{*}\right)>\omega\left(S ; \rho^{*} \underline{\rho}\right) .
$$

From Proposition 7.1 we know that the pair $\left[\omega\left(S ; \rho^{*} \mid \underline{\rho}^{*}\right), \omega\left(\underline{S} ; \rho^{*} \mid \underline{\rho}^{*}\right)\right]$ and $\left[\omega\left(S ; \rho^{*} \mid \underline{\rho}\right), \omega\left(\underline{S} ; \rho^{*} \mid \underline{\rho}\right)\right]$ are in the upper-right boundary $H_{S}$ of $F(S)$. Then we can immediately obtain that

$$
\omega\left(\underline{S} ; \rho^{*} \mid \underline{\rho}^{*}\right)<\omega\left(\underline{S} ; \rho^{*} \mid \underline{\rho}\right)
$$

which is in contradict with the rationality of the threat partition pair $\left(\rho^{*}, \underline{\rho}^{*}\right)$.

Then we can use the same proving process as above to verify that for any $\rho \in \Pi_{S}, \omega\left(\underline{S} ; \rho^{*} \mid \underline{\rho}^{*}\right) \leq \omega\left(\underline{S} ; \rho \mid \underline{\rho}^{*}\right)$.

Proof of Theorem 7.1. We have already seen in Example 7.2 that the RTPP may not exist, which implies that the RNBP may not exist. Next we only have to show the uniqueness of RNBP when there are multiple RTPPs.

We first show that for any two RTPPs $\left(\rho^{*}, \underline{\rho}^{*}\right),\left(\rho^{\prime}, \underline{\rho}^{\prime}\right), \omega\left(S ; \rho^{*} \mid \underline{\rho}^{*}\right)=$ $\omega\left(S ; \rho^{\prime} \mid \underline{\rho}^{\prime}\right)$. Let $\left(\rho^{*}, \underline{\rho}^{*}\right)$ and $\left(\rho^{\prime}, \rho^{\prime}\right)$ be two RTPPs. By using the rationality of $\left(\rho^{*}, \underline{\rho}^{*}\right)$ and Lemma 7.2 , we have that for any $\rho \in \Pi_{S}$ and any $\underline{\rho} \in \Pi_{\underline{S}}$

$$
\omega\left(S ; \rho \mid \underline{\rho}^{*}\right) \leq \omega\left(S ; \rho^{*} \mid \underline{\rho}^{*}\right) \leq \omega\left(S ; \rho^{*} \mid \underline{\rho}\right) .
$$

Similarly, it also holds that for any $\rho \in \Pi_{S}$ and any $\underline{\rho} \in \Pi_{\underline{S}}$

$$
\omega\left(S ; \rho \mid \underline{\rho}^{\prime}\right) \leq \omega\left(S ; \rho^{\prime} \mid \underline{\rho}^{\prime}\right) \leq \omega\left(S ; \rho^{\prime} \mid \underline{\rho}\right) .
$$

By using (7.1), (7.2), we have

$$
\omega\left(S ; \rho^{*} \mid \underline{\rho}^{*}\right) \leq \omega\left(S ; \rho^{*} \mid \underline{\rho}^{\prime}\right) \leq \omega\left(S ; \rho^{\prime} \mid \underline{\rho}^{\prime}\right) \leq \omega\left(S ; \rho^{\prime} \mid \underline{\rho}^{*}\right) \leq \omega\left(S ; \rho^{*} \mid \underline{\rho}^{*}\right) .
$$


Thus $\omega\left(S ; \rho^{*} \mid \underline{\rho}^{*}\right)=\omega\left(S ; \rho^{\prime} \mid \underline{\rho}^{\prime}\right)$.

Then we can use the same proving process as above to verify that for two $\operatorname{RTPPs}\left(\rho^{*}, \underline{\rho}^{*}\right)$ and $\left(\rho^{\prime}, \underline{\rho}^{\prime}\right), \omega\left(\underline{S} ; \rho^{*} \mid \underline{\rho}^{*}\right)=\omega\left(\underline{S} ; \rho^{\prime} \mid \underline{\rho}^{\prime}\right)$.

The following corollary can be easily deduced from the proof of Theorem 7.1.

Corollary 7.1. Let $(N, v)$ be a GPFG and $(N, u)$ the corresponding EPFG. Given any coalition $S \subseteq N$, if the two RTPPs $\left(\rho^{*}, \rho^{*}\right)$ and $\left(\rho^{\prime}, \rho^{\prime}\right)$ are rational, then so are $\left(\rho^{\prime}, \underline{\rho}^{*}\right)$ and $\left(\rho^{*}, \rho^{\prime}\right)$.

\subsection{Sufficient conditions for the existences of RTPPs}

In this section, we provide two sufficient conditions for the existence of RTPPs. In addition, we also investigate the exact forms of RTPPs in some special GPFGs. The first proposition provides a sufficient and necessary condition for every threat partition pair being rational.

Proposition 7.2. Let $(N, v)$ be a GPFG and $(N, u)$ the corresponding EPFG. For any coalition $S \subseteq N$, every threat partition pair with respect to $S$ is rational if and only if there is a pair of partitions $\left(\rho^{*}, \rho^{*}\right)$ such that for any $\rho \in \Pi_{S}$ and any $\underline{\rho} \in \Pi_{\underline{S}}$

$$
\begin{aligned}
& v\left(S ; \rho^{*} \mid \underline{\rho}^{*}\right) \geq v(S ; \rho \mid \underline{\rho}), \\
& v\left(\underline{S} ; \rho^{*} \mid \underline{\rho}^{*}\right) \geq v(\underline{S} ; \rho \mid \underline{\rho}) .
\end{aligned}
$$

Proof. The "if" part is obvious. Now we show the "only if" part. Suppose that there is no pair of partitions $\left(\rho^{*}, \underline{\rho}^{*}\right)$ satisfies for any $\rho \in \Pi_{S}$ and any $\underline{\rho} \in \Pi_{\underline{S}}$

$$
\begin{aligned}
& v\left(S ; \rho^{*} \mid \underline{\rho}^{*}\right) \geq v(S ; \rho \mid \underline{\rho}), \\
& v\left(\underline{S} ; \rho^{*} \mid \underline{\rho}^{*}\right) \geq v(\underline{S} ; \rho \mid \underline{\rho}) .
\end{aligned}
$$

Then it is easy to see that there must exist two threat partition pairs $\left(\rho^{1}, \rho^{1}\right)$, $\left(\rho^{2}, \underline{\rho}^{2}\right)$ such that $\left[v\left(S ; \rho^{1} \mid \underline{\rho}^{1}\right), v\left(\underline{S} ; \rho^{1} \mid \underline{\rho}^{1}\right)\right],\left[v\left(S ; \rho^{2} \mid \underline{\rho}^{2}\right), v\left(\underline{S} ; \rho^{2} \mid \underline{\rho^{2}}\right)\right]$ are 
both in the upper-right boundary $H_{S}$ of $F(S)$, and

$$
\left[v\left(S ; \rho^{1} \mid \underline{\rho}^{1}\right), v\left(\underline{S} ; \rho^{1} \mid \underline{\rho}^{1}\right)\right] \neq\left[v\left(S ; \rho^{2} \mid \underline{\rho}^{2}\right), v\left(\underline{S} ; \rho^{2} \mid \underline{\rho}^{2}\right)\right] .
$$

From Definition 7.5 and Proposition 7.1, it is obvious that

$$
\begin{gathered}
{\left[\omega\left(S ; \rho^{1} \mid \underline{\rho}^{1}\right), \omega\left(\underline{S} ; \rho^{1} \mid \underline{\rho}^{1}\right)\right]=\left[v\left(S ; \rho^{1} \mid \underline{\rho}^{1}\right), v\left(\underline{S} ; \rho^{1} \mid \underline{\rho}^{1}\right)\right],} \\
\quad\left[\omega\left(S ; \rho^{2} \mid \underline{\rho}^{2}\right), \omega\left(\underline{S} ; \rho^{2} \mid \underline{\rho}^{2}\right)\right]=\left[v\left(S ; \rho^{2} \mid \underline{\rho}^{2}\right), v\left(\underline{S} ; \rho^{2} \mid \underline{\rho}^{2}\right)\right] .
\end{gathered}
$$

Since $\left(\rho^{1}, \underline{\rho}^{1}\right)$ and $\left(\rho^{1}, \underline{\rho}^{2}\right)$ are both RTPPs, by using Theorem 7.1 and equation (7.4) we have

$$
\begin{aligned}
{\left[\omega\left(S ; \rho^{1} \mid \underline{\rho}^{2}\right), \omega\left(\underline{S} ; \rho^{1} \mid \underline{\rho}^{2}\right)\right] } & =\left[\omega\left(S ; \rho^{1} \mid \underline{\rho}^{1}\right), \omega\left(\underline{S} ; \rho^{1} \mid \underline{\rho}^{1}\right)\right] \\
& =\left[v\left(S ; \rho^{1} \mid \underline{\rho^{1}}\right), v\left(\underline{S} ; \rho^{1} \mid \underline{\rho}^{1}\right)\right] .
\end{aligned}
$$

Hence, we know that the Nash bargaining payoff derived from the threat partition pair $\left(\rho^{1}, \underline{\rho}^{2}\right)$ coincides with the point $\left[v\left(S ; \rho^{1} \mid \underline{\rho}^{1}\right), v\left(\underline{S} ; \rho^{1} \mid \underline{\rho^{1}}\right)\right]$ in the upper-right boundary.

Similarly, we can also obtain that

$$
\left[\omega\left(S ; \rho^{1} \mid \underline{\rho}^{2}\right), \omega\left(\underline{S} ; \rho^{1} \mid \underline{\rho}^{2}\right)\right]=\left[v\left(S ; \rho^{2} \mid \underline{\rho}^{2}\right), v\left(\underline{S} ; \rho^{2} \mid \underline{\rho}^{2}\right)\right] .
$$

The proof of (7.6) is similar to the proof of (7.5), so we omit it here. Then from (7.5), (7.6) we have

$$
\left[v\left(S ; \rho^{1} \mid \underline{\rho}^{1}\right), v\left(\underline{S} ; \rho^{1} \mid \underline{\rho}^{1}\right)\right]=\left[v\left(S ; \rho^{2} \mid \underline{\rho}^{2}\right), v\left(\underline{S} ; \rho^{2} \mid \underline{\rho}^{2}\right)\right],
$$

which contradicts (7.3).

Proposition 7.2 shows that coalitions $S$ and $\underline{S}$ will be indifferent to the threat partitions which they choose if and only if there is a pair of partitions under which the worths of the two coalitions are simultaneously maximized 
in a GPFG. The reason of obviousness of the "if" part in the proof of Proposition 7.2 is that $\left[v\left(S ; \rho^{*} \mid \underline{\rho}^{*}\right), v\left(\underline{S} ; \rho^{*} \mid \underline{\rho}^{*}\right)\right]$ is the only element in the upperright boundary $H_{S}$, which means that it is also the only Nash bargaining payoff.

Theorem 7.3. Let $(N, v)$ be a GPFG and $(N, u)$ the corresponding EPFG. For any coalition $S \subseteq N$, every threat partition pair with respect to $S$ is rational if

(i) $(N, v)$ has positive internalities and positive externalities, or

(ii) $(N, v)$ has negative internalities and negative externalities.

Proof. The proof is immediate from the definitions of positive (negative) internalities (externalities) and Proposition 7.2.

Our next proposition provides a sufficient condition for a threat partition pair to be rational. Before that, some notations and lemmas are required. Based upon the points of $H_{S}$, we denote the equation of $H_{S}$ on $F(S)$ as

$$
h_{S}\left(x_{1}, x_{2}\right)=0 \text {. }
$$

Since $F(S)$ is the convex hull enveloping all disagreement points, one can easily see that $h_{S}$ is a continuous and concave function. Moveover, for any $y \in$ $F(S), h_{S}(y) \geq 0$ while for any $y \in H_{S}, h_{S}(y)=0$. Let $y \in H_{S}$, we define the left (right) partial derivative of $h_{S}$ with respect to $x_{1}$ at $y_{1}$ as

$$
\left.\frac{\partial h_{S}}{\partial x_{1}}\right|_{\left(y_{1}, y_{2}\right)^{-(+)}}=\lim _{\epsilon \rightarrow 0^{-(+)}} \frac{h_{S}\left(y_{1}+\epsilon, y_{2}\right)-h_{S}\left(y_{1}, y_{2}\right)}{\epsilon} .
$$

A graphic description is given in Figure 7.7, where the $u_{1}$-axis and the $u_{2}$-axis represent the payoff for coalitions $S$ and $\underline{S}$ respectively. Clearly for $y$, the left partial derivative of $h_{S}$ with respect to $x_{1}$ at $y_{1}$ equals to the slope of the line segment $A B$, and the right partial derivative of $h_{S}$ with respect to $x_{1}$ at $y_{1}$ equals to the slope of the line segment $A C$. 


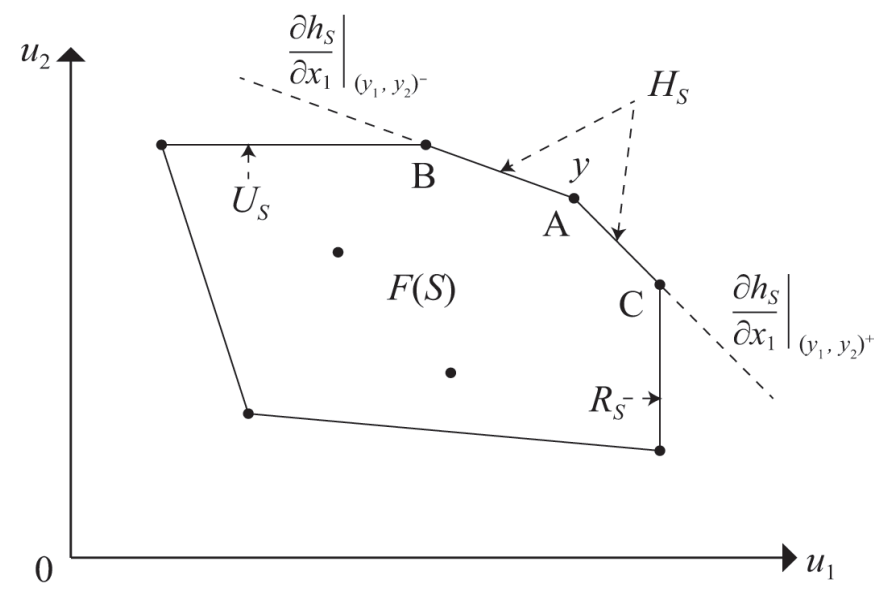

FIGURE 7.7: A graphic description about the left (right) partial derivative.

Lemma 7.4. Let $(N, v)$ be a GPFG and $(N, u)$ the corresponding EPFG. Given any coalition $S \subseteq N$ and the associated equation $h_{S}$ of the upper-right boundary $H_{S}$, if for any $y, z \in H_{S}$ with $y_{1} \leq z_{1}$, then we have

$$
\left.\frac{\partial h_{S}}{\partial x_{1}}\right|_{\left(y_{1}, y_{2}\right)^{+}} \geq\left.\frac{\partial h_{S}}{\partial x_{1}}\right|_{\left(z_{1}, z_{2}\right)^{-}} .
$$

Proof. The fact that $F(S)$ is a convex hull formed by discrete points implies that $h_{S}$ is a continuous, concave and piecewise linear function of the upperright boundary $H_{S}$. We denote the linear functions on different segments of $h_{S}$ by $h_{S}^{1}, \ldots, h_{S}^{m+1}$ respectively. The vertexes, which are the points of intersection of two linear functions, are denoted by $\alpha^{1}, \ldots, \alpha^{m}$, where $\alpha_{1}^{1} \leq \ldots \leq \alpha_{1}^{m}$. For any vertex $\alpha^{i}(i=1, \ldots, m)$, the concavity of $h_{S}$ ensures that

$$
\left.\frac{\partial h_{S}}{\partial x_{1}}\right|_{\left(\alpha_{1}^{i}, \alpha_{2}^{i}\right)^{-}}>\left.\frac{\partial h_{S}}{\partial x_{1}}\right|_{\left(\alpha_{1}^{i}, \alpha_{2}^{i}\right)^{+}} .
$$

Moreover, it is not hard to see that for any $1 \leq i \leq m-1$, the vertexes $\alpha^{i}$ and $\alpha^{i+1}$ are on the same linear function which is one of the linear functions 
constituting $h_{S}$. Hence we have,

$$
\left.\frac{\partial h_{S}}{\partial x_{1}}\right|_{\left(\alpha_{1}^{i}, \alpha_{2}^{i}\right)^{+}}=\left.\frac{\partial h_{S}}{\partial x_{1}}\right|_{\left(\alpha_{1}^{i+1}, \alpha_{2}^{i+1}\right)^{-}} .
$$

Use (7.7), (7.8), we obtain for any $1 \leq i<j \leq m$

$$
\left.\frac{\partial h_{S}}{\partial x_{1}}\right|_{\left(\alpha_{1}^{i}, \alpha_{2}^{i}\right)^{+}} \geq\left.\frac{\partial h_{S}}{\partial x_{1}}\right|_{\left(\alpha_{1}^{j}, \alpha_{2}^{j}\right)^{-}} .
$$

Next, we consider the points on $h_{S}$ which are not vertexes. Let $y, z \in$ $H_{S}$ be two points which are not vertexes and satisfy $y_{1} \leq z_{1}$. Let $\alpha^{i}$ be the vertex nearest to $y$ with $\alpha_{1}^{i}>y_{1}$ and $\alpha^{j}$ be the vertex nearest to $z$ with $\alpha_{1}^{j}>z_{1}$. Clearly, $i \leq j$. If $y, z$ are on the same linear function, it is obvious that Lemma 7.4 is true. If $y, z$ on the different linear functions of $h_{S}$,

$$
\left.\frac{\partial h_{S}}{\partial x_{1}}\right|_{\left(y_{1}, y_{2}\right)^{+}}=\left.\frac{\partial h_{S}}{\partial x_{1}}\right|_{\left(\alpha_{1}^{i}, \alpha_{2}^{i}\right)^{-}}>\left.\frac{\partial h_{S}}{\partial x_{1}}\right|_{\left(\alpha_{1}^{j}, \alpha_{2}^{j}\right)^{-}}=\left.\frac{\partial h_{S}}{\partial x_{1}}\right|_{\left(z_{1}, z_{2}\right)^{+}}=\left.\frac{\partial h_{S}}{\partial x_{1}}\right|_{\left(z_{1}, z_{2}\right)^{-}},
$$

where the inequality follows from (7.7) and (7.9). The first and the second equalities follow from the fact that $y$ is on the same linear function with $\alpha^{i}$ and $z$ is on the same linear function with $\alpha^{j}$. Since $z$ is not a vertex, the last equality certainly holds.

Lemma 7.5. Let $(N, v)$ be a GPFG and $(N, u)$ the corresponding EPFG. Given any $S \subseteq N$, let $d \in F(S) \backslash\left(U_{S} \cup R_{S} \cup H_{S}\right)$ be a disagreement point. Then $y \in H_{S}$ is the Nash bargaining payoff if and only if there are some $\lambda \in \mathbb{R}$ such that

$$
\lambda=-\frac{y_{2}-d_{2}}{y_{1}-d_{1}} \text { and }\left.\frac{\partial h_{S}}{\partial x_{1}}\right|_{\left(y_{1}, y_{2}\right)^{+}} \leq \lambda \leq\left.\frac{\partial h_{S}}{\partial x_{1}}\right|_{\left(y_{1}, y_{2}\right)^{-}} .
$$

Proof. The proof of this lemma can be immediately deduced from Theorem 8.2 in [64].

Proposition 7.3. Let $(N, v)$ be a GPFG and $(N, u)$ the corresponding EPFG. For any coalition $S \subseteq N$, a threat partition pair $\left(\rho^{*}, \underline{\rho}^{*}\right)$ is rational if for any 
$\rho \in \Pi_{S}$

$$
\begin{aligned}
& v\left(S ; \rho^{*} \mid \underline{\rho}^{*}\right) \geq v\left(S ; \rho \mid \underline{\rho}^{*}\right), \\
& v\left(\underline{S} ; \rho^{*} \mid \underline{\rho}^{*}\right) \leq v\left(\underline{S} ; \rho \mid \underline{\rho}^{*}\right),
\end{aligned}
$$

and for any $\underline{\rho} \in \Pi_{\underline{S}}$

$$
\begin{aligned}
& v\left(S ; \rho^{*} \mid \underline{\rho}^{*}\right) \leq v\left(S ; \rho^{*} \mid \underline{\rho}\right), \\
& v\left(\underline{S} ; \rho^{*} \mid \underline{\rho}^{*}\right) \geq v\left(\underline{S} ; \rho^{*} \mid \underline{\rho}\right) .
\end{aligned}
$$

Proof. It is sufficient to show that for any $\rho \in \Pi_{S}$ and any $\underline{\rho} \in \Pi_{\underline{S}}$,

$$
\begin{aligned}
& \omega\left(S ; \rho^{*} \mid \underline{\rho}^{*}\right) \geq \omega\left(S ; \rho \mid \underline{\rho}^{*}\right), \\
& \omega\left(\underline{S} ; \rho^{*} \mid \underline{\rho}^{*}\right) \geq \omega\left(\underline{S} ; \rho^{*} \mid \underline{\rho}\right) .
\end{aligned}
$$

First, we need to prove that (7.10) is true. Suppose that there is some $\rho \in \Pi_{S}$ such that

$$
\omega\left(S ; \rho^{*} \mid \underline{\rho}^{*}\right)<\omega\left(S ; \rho \mid \underline{\rho}^{*}\right) .
$$

Then from Proposition 7.1, we must have

$$
\omega\left(\underline{S} ; \rho^{*} \mid \underline{\rho}^{*}\right)>\omega\left(\underline{S} ; \rho \mid \underline{\rho}^{*}\right) .
$$

For notational convenience and with little confusion, we denote the points $\left[\omega\left(S ; \rho^{*} \mid \underline{\rho}^{*}\right), \omega\left(\underline{S} ; \rho^{*} \mid \underline{\rho}^{*}\right)\right],\left[v\left(S ; \rho^{*} \mid \underline{\rho}^{*}\right), v\left(\underline{S} ; \rho^{*} \mid \underline{\rho}^{*}\right)\right],\left[\omega\left(S ; \rho \mid \underline{\rho}^{*}\right), \omega\left(\underline{S} ; \rho \mid \underline{\rho}^{*}\right)\right]$ and $\left[v\left(S ; \rho \mid \underline{\rho}^{*}\right), v\left(\underline{S} ; \rho \mid \underline{\rho}^{*}\right)\right]$ by $\omega *, v *, \omega$, and $v$ respectively.

Case 1: $v^{*} \in H_{S}$. Then clearly $v^{*}=\omega^{*}$. Thus, we have $\omega_{2} \geq v_{2} \geq v_{2}^{*}=$ $\omega_{2}^{*}$. This contradicts (7.13).

Case 2: $v^{*} \in U_{S}$. Then it is easy to verify that $v_{2}^{*}=\omega_{2}^{*}$. Thus we have $\omega_{2} \geq v_{2} \geq v_{2}^{*}=\omega_{2}^{*}$, which also contradicts (7.13).

Case 3: $v^{*} \in R_{S}$. Then it is easy to verify that $v_{1}^{*}=\omega_{1}^{*}$. Thus we have $\omega_{1}^{*}=v_{1}^{*} \geq \omega_{1}$, which is in contraction with (7.12). 
Case 4: $v^{*} \in F(S) \backslash\left(U_{S} \cup R_{S} \cup H_{S}\right)$. Obviously in this case, $v \in F(S) \backslash H_{S}$. Besides, we can surely get

$$
\frac{\omega_{2}^{*}-v_{2}^{*}}{\omega_{1}^{*}-v_{1}^{*}}>\frac{\omega_{2}-v_{2}}{\omega_{1}-v_{1}}
$$

Then by Lemma 7.5 we know that there are some $\lambda^{*}, \lambda \in \mathbb{R}$ such that

$$
\begin{gathered}
\lambda^{*}=-\frac{\omega_{2}^{*}-v_{2}^{*}}{\omega_{1}^{*}-v_{1}^{*}} \text { and }\left.\frac{\partial h_{S}}{\partial x_{1}}\right|_{\left(\omega_{1}^{*}, \omega_{2}^{*}\right)^{+}} \leq \lambda^{*} \leq\left.\frac{\partial h_{S}}{\partial x_{1}}\right|_{\left(\omega_{1}^{*}, \omega_{2}^{*}\right)^{-}}, \\
\lambda=-\frac{\omega_{2}-v_{2}}{\omega_{1}-v_{1}} \text { and }\left.\frac{\partial h_{S}}{\partial x_{1}}\right|_{\left(\omega_{1}, \omega_{2}\right)^{+}} \leq \lambda \leq\left.\frac{\partial h_{S}}{\partial x_{1}}\right|_{\left(\omega_{1}, \omega_{2}\right)^{-}} .
\end{gathered}
$$

Use (7.14), (7.15) and (7.16) to obtain

$$
\left.\frac{\partial h_{S}}{\partial x_{1}}\right|_{\left(\omega_{1}^{*}, \omega_{2}^{*}\right)^{+}} \leq \lambda^{*}<\lambda \leq\left.\frac{\partial h_{S}}{\partial x_{1}}\right|_{\left(\omega_{1}, \omega_{2}\right)^{-}},
$$

which is in contradiction with Lemma 7.4.

The proof of (7.11) is similar to the proof of (7.10), so we omit it here.

Proposition 7.3 shows that a threat partition pair $\left(\rho^{*}, \rho^{*}\right)$ must be an RTPP if, first, $S$ or $\underline{S}$ changes its partition, given that the other one sticks to the rational threat, its payoff when the new threat partition pair is implemented is not better than that when $\left(\rho^{*}, \underline{\rho}^{*}\right)$ is implemented; and second, the payoff for the other one when the new threat partition pair is implemented is not worse than that when $\left(\rho^{*}, \underline{\rho}^{*}\right)$ is implemented.

Theorem 7.6. Let $(N, v)$ be a GPFG and $(N, u)$ the corresponding EPFG.

(i) If $(N, v)$ has positive internalities and negative externalities, then for any $S \subseteq N$ the threat partition pair $(\{S\},\{\underline{S}\})$ is rational.

(ii) If $(N, v)$ has negative internalities and positive externalities, then for any $S \subseteq N$ the threat partition pair $([S],[\underline{S}])$ is rational.

Proof. The proof is immediate from the definitions of positive (negative) internalities (externalities) and Proposition 7.3. 


\subsection{The convexity and the core}

In this section, we focus on the GPFGs in which for any $S \subseteq N$ there exists at least one RTPP with respect to $S$. We denote this kind of GPFGs by $G^{n}$.

Definition 7.7. Let $(N, v) \in G^{n}$ and $(N, u)$ be the corresponding EPFG. The corresponding Nash rational bargaining game (NRBG) is a pair $\left(N, v^{u}\right)$, where $N$ is the set of players and $v^{u}: 2^{N} \rightarrow \mathbb{R}$ is a map that assigns to each coalition $S \subseteq N$ the value

$$
v^{u}(S)=\omega\left(S ; \rho^{*} \mid \underline{\rho}^{*}\right),
$$

where $\left(\rho^{*}, \rho^{*}\right)$ is an RTPP.

Because of the uniqueness of RNBP which has been proved in Theorem 7.1, for any $(N, v) \in G^{n}$ and any $S \subseteq N, v^{u}(S)$ is well-defined.

Next, we want to investigate the fair allocations of the "surplus" of the grand coalition in the situation that all players decide to cooperate. A natural thought is that this "surplus" should be equal to the maximal worth of the grand coalition under some partition of $N$, i.e., $\max _{\mathcal{N} \in \Pi_{N}} v\left(N ; \rho_{N} \mid \emptyset\right)$. Coincidentally, it can be easily verified that $v^{u}(N)=\max _{\rho_{N} \in \Pi_{N}} v\left(N ; \rho_{N} \mid \emptyset\right)$. Thus, by assuming that every coalition considers its RNBP, i.e., $v^{u}(S)$ as its final worth, we define the set of allocations that makes no coalition get less than $v^{u}(S)$. The allocations satisfying these conditions form the core of a $\left(N, v^{u}\right)$, which are given in the next definition.

With abuse of notation, we denote the allocation vector as $x=\left(x_{1}, . ., x_{n}\right) \in$ $\mathbb{R}^{n}$. For notational simplicity we denote $\sum_{i \in S} x_{i}$ by $x(S)$.

Definition 7.8. Let $(N, v) \in G^{n}$ and $\left(N, v^{u}\right)$ be the corresponding $N R B G$. The core of $\left(N, v^{u}\right)$ is defined as

$$
C\left(v^{u}\right)=\left\{x \in \mathbb{R}^{n} \mid x(S) \geq v^{u}(S) \text { for any } S \subseteq N\right\} .
$$

In the rest of this section, we show two sufficient conditions for the nonemptiness of $C\left(v^{u}\right)$. Before that, we need some additional notations. For any $S, T \subseteq N$ and any $\rho$ of $S, \rho^{\prime}$ of $T$, define

$$
\rho \wedge \rho^{\prime}=\left\{S^{\prime} \cap T^{\prime} \mid S^{\prime} \in \rho, T^{\prime} \in \rho^{\prime}, S^{\prime} \cap T^{\prime} \neq \emptyset\right\} .
$$


The following definition provides a concept of convexity for GPFGs, which is an extension of the convexity of PFF games defined by Hafalir [36].

Definition 7.9. A GPFG is convex if for any $S, T \subseteq N$, any partition $\rho_{1}$ of $S \cup T$ and any partition $\rho_{2}$ of $N \backslash(S \cup T)$,

$$
\begin{aligned}
& v\left(S \cup T, \rho_{1} \mid \rho_{2}\right)+v\left(S \cap T, \rho_{c} \mid \rho_{2} \cup\{S \backslash T\} \cup\{T \backslash S\}\right) \\
\geq & v\left(S ; \rho_{a} \mid \rho_{2} \cup\{T \backslash S\}\right)+v\left(T, \rho_{b} \mid \rho_{2} \cup\{S \backslash T\}\right),
\end{aligned}
$$

where $\rho_{a}=\rho_{1} \wedge\{S\}, \rho_{b}=\rho_{1} \wedge\{T\}$ and $\rho_{c}=\rho_{a} \wedge \rho_{b}$.

Theorem 7.7. Let $(N, v) \in G^{n}$ and $\left(N, v^{u}\right)$ be the corresponding $N R B G$. If $(N, v)$ is convex and

(i) has positive internalities and negative externalities, then $C\left(v^{u}\right) \neq \emptyset$.

(ii) has negative internalities and negative externalities, then $C\left(v^{u}\right) \neq \emptyset$.

Proof. (i) When we fix $\rho_{1}=\{S \cup T\}$ in Definition 7.9, the convexity of a GPFG coincides with the convexity of a PFF game. Then from Proposition 2 of [36], there is a $x \in \mathbb{R}^{n}$ such that for any $S \subseteq N$,

$$
x(S) \geq v(S ;\{S\} \mid[\underline{S}]) .
$$

Since $(N, v)$ has positive internalities and negative externalities, then we have

$$
v(S ;\{S\} \mid[\underline{S}])=\max _{\substack{\rho \in \Pi_{S} \\ \underline{\rho} \in \Pi_{\underline{S}}}} v(S ; \rho \mid \underline{\rho}) \geq \omega(S ;\{S\} \mid\{\underline{S}\}) .
$$

By using (7.17) and (7.18) we have for any $S \subseteq N$,

$$
x(S) \geq \omega(S ;\{S\} \mid\{\underline{S}\})=v^{u}(S),
$$

where the equality follows from Theorem 7.6.

(ii) Define the CFF game $(N, V)$ with $V(S)=v(S ;[S] \mid[\underline{S}])$. In the following, we show that $(N, V)$ is convex. 
Fix $\rho_{1}=[S \cup T]$ and $\rho_{2}=[N \backslash(S \cup T)]$ in Definition 7.9, then for any $S, T \subseteq$ $N$ we have

$$
\begin{aligned}
& \quad v(S \cup T,[S \cup T] \mid[N \backslash(S \cup T)]) \\
& \quad+v(S \cap T,[S \cap T] \mid[N \backslash(S \cup T)] \cup\{S \backslash T\} \cup\{T \backslash S\}) \\
& \geq v(S ;[S] \mid[N \backslash(S \cup T)] \cup\{T \backslash S\})+v(T,[T] \mid[N \backslash(S \cup T)] \cup\{S \backslash T\}) .
\end{aligned}
$$

If we take any $S, T \subseteq N$ with $|T \backslash S|=|S \backslash T|=1$, then (7.19) can be rewritten as

$$
V(S \cup T)+V(S \cap T) \geq V(S)+V(T) .
$$

Moulin [61] showed that the weak convexity is equivalent to convexity of CFF games, which means that without the restriction " $|T \backslash S|=|S \backslash T|=1$ ", (7.20) is also true. Because the core of a convex CFF game must be nonempty [78], we obtain that there must be $x \in \mathbb{R}^{n}$ such that for any $S \subseteq N$

$$
x(S) \geq v(S ;[S] \mid[\underline{S}]) .
$$

Since $(N, v)$ has negative internalities and negative externalities, from Theorem 7.3 we know that any threat partition pair $(\rho, \rho)$ is rational. Moreover, it is clear that for any $\rho \in \Pi_{S}$ and any $\underline{\rho} \in \Pi_{\underline{S}}$

$$
[v(S ;[S] \mid[\underline{S}]), v(\underline{S} ;[S] \mid[\underline{S}])]=[\omega(S ; \rho \mid \underline{\rho}), \omega(\underline{S} ; \rho \mid \underline{\rho})] .
$$

From (7.21), (7.22) we conclude that for any $\rho \in \Pi_{S}$ and any $\underline{\rho} \in \Pi_{\underline{S}}$

$$
x(S) \geq \omega(S ; \rho \mid \underline{\rho})=v^{u}(S)
$$

\subsection{Concluding remarks}

\subsubsection{Comparisons between our games and PFF games}

We have introduced a new game, the general partition function game, in which the worth of a coalition not only depends on its internal coalition 
structure but also the partition of external players. Note that a GPFG will be reduced to a PFF game under the condition that the internal structure of any deviating coalition $S$ is forced to be $\{S\}$ by some compulsory rule. In other words, a PFF game only takes externalities into account, but not the internalities.

The same idea of considering internalities in the analysis of coalitional function also appears in [8] and [10]. They all point out that a coalition will reorganize its own partition when it separates from the grand coalition, and the final payoff for this coalition relies on both the internal rearrangement and the reaction of external players. Although the spirit is the same, the coalitional function games defined in their paper are rooted in PFf games (the worth of a coalition $S$ is the sum of the surplus of the atoms of $S$, i.e., $\left.\sum_{T \in \rho} f(T ; \rho \cup \underline{\rho})\right)$, which can be seen as special forms of GPFGs.

In the model we defined in Section 7.3, a coalition evaluates its worth by the measure of its RNBP (if exists). Given any coalition $S$, the RNBP for this coalition actually is an expected worth generated by some mixed partition pair, since it is in the feasible payoff set $F(S)$. This is quite different from the measurement methods for a deviating coalition in most the previous studies which generally consider that the partitions of internal players and external players should be deterministic. Because of the piecewise linearity of the upper-right boundary, one can easily verify that, in our model, the RNBP $v^{u}(S)$ for a coalition $S$ is a convex combination of two worths of $S$ based on two pairs of partitions. Formally, suppose that $\left(\rho^{*}, \rho^{*}\right)$ is an RTPP, then there must exist two pairs of partitions $(\rho, \underline{\rho}),\left(\rho^{\prime}, \underline{\rho}^{\prime}\right)$ such that for some $0 \leq \lambda \leq 1$,

$$
v^{u}(S)=\omega\left(S ; \rho^{*} \mid \underline{\rho}^{*}\right)=\lambda v(S ; \rho \mid \underline{\rho})+(1-\lambda) v\left(S ; \rho^{\prime} \mid \underline{\rho}^{\prime}\right) .
$$

This conclusion can also be supported by the observation of Figure 7.2.

\subsubsection{Alternative models}

We introduce three other models in this subsection. The first model is inspired by Shenoy [79] in which the external players partition into the best-case scenario for the deviating coalition. In other words, the deviating coalition is 
very optimistic and expects the residual players to act in an extremely generous way as to benefit deviators to the maximum extent. Formally, we define

$$
v^{o}(S)=\max _{\hat{\rho}} \max _{\underline{\hat{\rho}}} u(S ; \hat{\rho} \mid \underline{\hat{\rho}}) .
$$

The following two models originate from the maximin-minimax rule proposed by Von Neumann and Morgenstern [86] and can also be regarded as an inspiration from the definitions of the $\alpha$-core and $\beta$-core in [2]. Formally, we define

$$
\begin{aligned}
v^{\alpha}(S) & =\max _{\hat{\rho}} \min _{\hat{\rho}} u(S ; \hat{\rho} \mid \underline{\hat{\rho}}), \\
v^{\beta}(S) & =\min _{\underline{\hat{\rho}}} \max _{\hat{\rho}} u(S ; \hat{\rho} \mid \underline{\hat{\rho}}) .
\end{aligned}
$$

The basic similarity of the above two definitions is that the deviating coalition is very pessimistic and expects the outsiders to partition into the worst case scenario for the deviators. In (7.24) coalition $S$ knows that for any its mixed partition, the residual players will react to hurt deviators as much as possible. So coalition $S$ takes the strategy which can guarantee the maximal payoff $v^{\alpha}(S)$ for itself. Whereas in (7.25), for any mixed partition of $\underline{S}$, coalition $S$ react to maximize its own payoff and in this case coalition $\underline{S}$ will certainly choose a strategy minimizing the ex-post payoff of $S$. It can also be interpreted as a situation that coalition $\underline{S}$ can not prevent $S$ from getting $v^{\beta}(S)$. One can easily verify $v^{\beta}(S)=v^{\alpha}(S)$ according to the Minimax theory proposed by Von Neumann [85].

The expectation for the formation of outsiders, whether is optimistic or pessimistic, underlines a very strong assumption that the residual players never think about their own benefit and always act in an extreme way. The Nash bargaining model that is defined in Section 7.3 drops this assumption and we are focusing on a situation that leads to a win-win result. In this way, the final payoff for the deviating coalition obtained by bilateral cooperating and bargaining is more reasonable than by the behavioral assumptions of optimism and pessimism. Moreover, it is easy to see that $v^{\alpha}(S)=v^{\beta}(S) \leq$ 
$v^{u}(S) \leq v^{o}(S)$. Thus, the following proposition requires no proof.

Proposition 7.4. Let $(N, v) \in G^{n}$ and $\left(N, v^{u}\right)$ be the corresponding NRBG.

Then we have

$$
C\left(v^{o}\right) \subseteq C\left(v^{u}\right) \subseteq C\left(v^{\beta}\right)=C\left(v^{\alpha}\right) .
$$




\section{Summary}

In this thesis, we present solutions for different sequencing and bargaining situations. We first consider cooperative sequencing games in more general cases, such as scheduling with learning effect and externalities. We then provide a noncooperative bargaining game and show that the stationary subgame perfect equilibrium payoff profiles of these games coincide with the core. Moreover, we study a general coalitional bargaining game where the approval of a proposal follows a majority rule. We show that stationary subgame perfect equilibria in these games exist. Finally, we propose a class of general partition function games and show that there is at most one rational Nash bargaining payoff.

In Chapter 3, we follow the work of Curiel et al. [21] to investigate the solutions in sequencing situations with position-dependent learning effect by using cooperative game theory. The main result shows that the sequencing game with learning effect has a nonempty core and we provide an algorithm to compute a unique equal gain splitting allocation of the maximum cost savings in the core. One restriction of the model is that the weights of players are assumed to be equal and thus w.l.o.g. they are assumed to be 1. We ask the question of how to build cooperative sequencing games with learning effect and with different weights of players. In this situation, one major problem still open is to find an optimal order. Unfortunately, we do not answer this question in this thesis. We discuss the complexity of computing an optimal order in scheduling problems with learning effect and with different weights of players, and conjecture that they are NP-hard. If our conjecture is correct, then there will be not much hope for using the same cooperative approach as we did for the sequencing situation with learning effect and identical weights 
of players. To solve that problem is left as future work.

In Chapter 4, we have extended the cooperative games based on the sequencing situations to partition function form. The intuition behind partition sequencing games is that the worth of a deviating coalition can be influenced by the coalition structure of external players in the queue. The first contribution of this chapter is that we show that partition sequencing games satisfy many natural properties such as possess cohesiveness (the grand coalition generates the largest total surplus) and non-positive externalities (the merger between two disjoint coalitions does not make other coalitions better off). We find that the EGS rule always yields a core element no matter which partition rule is applied to the games. The second contribution of this chapter is the implementation of the EGS rule in partition sequencing games. The mechanism we propose in this chapter is similar to that of [7], [15], and [73], but different in two ways: first, the discounting factor is not incorporated into the dynamic game; and second, the proposer proposing a proposal that is certainly rejected will certainly be removed from the game.

One may find that the above two research chapters do not impose further restrictions like due dates, release dates, or precedence relations into sequencing situations. It will be interesting for us to see what will happen if we add these assumptions into our settings.

Chapter 5 proposes a coalitional bargaining model in which the protocol is endogenously determined by the players. Additionally, we embed a new concept called the partial breakdown probability in the games, which can be interpreted as the rejector's personal prediction for the possibility of the departure of the coalition being proposed. The main result is that the outcomes of stationary subgame perfect equilibria coincide with the core allocations if the characteristic function is totally balanced. Unlike the assumption that proposers are determined by external factors such as random selection rule or sequential selection rule that are usually made in the literature, the endogenous selection of proposers in our model is a more fair rule to the players, as the selection of a proposer only depends on the strategies of the players. On the other hand, our model can be considered more general than that in [77], since our bargaining game may involve multiple periods, and a rejected coalition will not be immediately kicked out of the game. Instead, the staying of 
the rejected coalition is determined by the partial breakdown probability.

Chapter 6 considers coalitional bargaining games with majority rule. These games are more general than those games defined by Okada [68]. The main generalization is that the approval of a proposal does not necessarily need a unanimous consent of all members in the proposal coalition, but only part of the coalition is sufficient. The main conclusion shows that stationary subgame perfect equilibria exist in that class of games. One basic assumption of the underlying characteristic function is superadditivity. This assumption indicates that forming the grand coalition will be most efficient for all players. Therefore, we focus on grand-coalition efficient games for analyzing properties of expected equilibrium payoffs. We show that the pivotal players extract all the gains of the grand coalition in this class of games. However, one important question that still needs to be answered is what necessary and sufficient conditions are for the existence of grand-coalition efficient games. We answer the question when all players in the game are symmetric. To answer the question in general situations, we need future work.

Chapter 7 considers a class of general partition function games, in which the worth of a coalition is both influenced by internalities and externalities. The partition function we define in this chapter is more general than PFF games in the sense that the worth of a coalition is also determined by the partition of its members. A Nash bargaining model is applied to determine the reasonable worths of coalitions. We show that there is at most one rational Nash bargaining payoff for any deviating coalition. Although we provide two sufficient conditions for the existence of rational threat partition pairs, the necessary and sufficient conditions are still unknown. This requires future work. 



\section{Bibliography}

[1] T. Abe and Y. Funaki. The non-emptiness of the core of a partition function form game. International Journal of Game Theory, pages 1-22, 2016.

[2] R. J. Aumann and B. Peleg. Von neumann-morgenstern solutions to cooperative games without side payments. Bulletin of the American Mathematical Society, 66:173-179, 1960.

[3] D. P. Baron and J. A. Ferejohn. Bargaining in legislatures. American political science review, 83(4):1181-1206, 1989.

[4] C. Berge. Espaces topologiques, fonctions multivoques, volume 3. Dunod, 1966.

[5] D. Biskup. Single-machine scheduling with learning considerations. European Journal of Operational Research, 115(1):173-178, 1999.

[6] D. Biskup. A state-of-the-art review on scheduling with learning effects. European Journal of Operational Research, 188(2):315-329, 2008.

[7] F. Bloch. Sequential formation of coalitions in games with externalities and fixed payoff division. Games and Economic Behavior, 14(1):90-123, 1996.

[8] F. Bloch and A. Van den Nouweland. Expectation formation rules and the core of partition function games. Games and Economic Behavior, 88:339-353, 2014. 
[9] P. Borm, G. Fiestras-Janeiro, H. Hamers, E. Sánchez, and M. Voorneveld. On the convexity of games corresponding to sequencing situations with due dates. European Journal of Operational Research, 136(3):616-634, 2002.

[10] P. Borm, Y. Ju, and D. Wettstein. Rational bargaining in games with coalitional externalities. Journal of Economic Theory, 157:236-254, 2015.

[11] L. E. J. Brouwer. Über abbildung von mannigfaltigkeiten. Mathematische annalen, 71(1):97-115, 1911.

[12] P. Calleja, P. Borm, H. Hamers, F. Klijn, and M. Slikker. On a new class of parallel sequencing situations and related games. Annals of Operations Research, 109(1-4):265-277, 2002.

[13] P. Chander and H. Tulkens. The core of an economy with multilateral environmental externalities. International Journal of Game Theory, 3(26):379-401, 1997.

[14] C. Chang and C.-C. Hu. A non-cooperative interpretation of the kernel. International Journal of Game Theory, 46(1):185-204, 2017.

[15] K. Chatterjee, B. Dutta, D. Ray, and K. Sengupta. A noncooperative theory of coalitional bargaining. The Review of Economic Studies, 60(2):463-477, 1993.

[16] T. E. Cheng and G. Wang. Single machine scheduling with learning effect considerations. Annals of Operations Research, 98(1-4):273-290, 2000.

[17] B. Çiftçi, P. Borm, H. Hamers, and M. Slikker. Batch sequencing and cooperation. Journal of Scheduling, 16(4):405-415, 2013.

[18] I. Curiel. Multi-stage sequencing situations. International Journal of Game Theory, 39:151-162, 2010.

[19] I. Curiel. Compensation rules for multi-stage sequencing games. Annals of Operations Research, 225(1):65-82, 2015. 
[20] I. Curiel, H. Hamers, and F. Klijn. Sequencing games: a survey. In Chapters in game theory, pages 27-50. Springer, 2002.

[21] I. Curiel, G. Pederzoli, and S. Tijs. Sequencing games. European Journal of Operational Research, 40(3):344-351, 1989.

[22] I. Curiel, J. Potters, R. Prasad, S. Tijs, and B. Veltman. Cooperation in one machine scheduling. Zeitschrift für Operations Research, 38(2):113129, 1993.

[23] I. Curiel, J. Potters, R. Prasad, S. Tijs, and B. Veltman. Sequencing and cooperation. Operations Research, 42(3):566-568, 1994.

[24] G. De Clippel and R. Serrano. Marginal contributions and externalities in the value. Econometrica, 76:1413-1436, 2008.

[25] B. Dutta, L. Ehlers, and A. Kar. Externalities, potential, value and consistency. Journal of Economic Theory, 145:2380-2411, 2010.

[26] D. W. Engels, D. R. Karger, S. G. Kolliopoulos, S. Sengupta, R. Uma, and J. Wein. Techniques for scheduling with rejection. Journal of Algorithms, 49(1):175-191, 2003.

[27] H. Eraslan. Uniqueness of stationary equilibrium payoffs in the baronferejohn model. Journal of Economic Theory, 103(1):11-30, 2002.

[28] R. Evans. Coalitional bargaining with competition to make offers. Games and Economic Behavior, 19(2):211-220, 1997.

[29] Y. Funaki and T. Yamato. The core of an economy with a common pool resource: A partition function form approach. International Journal of Game Theory, 28:157-171, 1999.

[30] M. Gerichhausen and H. Hamers. Partitioning sequencing situations and games. European Journal of Operational Research, 196(1):207-216, 2009.

[31] D. Gillies. Some theorems on n-person games. Ph. D. Dissertation, Princeton University, Department of Mathematics, 1953. 
[32] A. Gomes. Multilateral contracting with externalities. Econometrica, 73(4):1329-1350, 2005.

[33] A. Gomes. Multilateral negotiations and formation of coalitions. Journal of Mathematical Economics, 59:77-91, 2015.

[34] J. Greenberg. Coalition structures. Handbook of game theory with economic applications, 2:1305-1337, 1994.

[35] S. Grundel, B. Çiftçi, P. Borm, and H. Hamers. Family sequencing and cooperation. European Journal of Operational Research, 226(3):414424, 2013.

[36] I. E. Hafalir. Efficiency in coalition games with externalities. Games and Economic Behavior, 61:242-258, 2007.

[37] N. G. Hall and Z. Liu. Capacity allocation games without an initial sequence. Operations Research Letters, 44(6):747-749, 2016.

[38] H. Hamers, P. Borm, and S. Tijs. On games corresponding to sequencing situations with ready times. Mathematical Programming, 69(1-3):471483, 1995.

[39] H. Hamers, F. Klijn, and J. Suijs. On the balancedness of multiple machine sequencing games. European Journal of Operational Research, 119(3):678-691, 1999.

[40] J. C. Harsanyi. A simplified bargaining model for the n-person cooperative game. International Economic Review, 4(2):194-220, 1963.

[41] S. Hart and M. Kurz. Endogenous formation of coalitions. Econometrica: Journal of the Econometric Society, 51:1047-1064, 1983.

[42] S. Hart and A. Mas-Colell. Bargaining and value. Econometrica: Journal of the Econometric Society, pages 357-380, 1996.

[43] M. Herrero. A strategic bargaining approach to market institutions. Unpublished Ph. D. Thesis. University of London, 1985. 
[44] C.-Y. Huang and T. Sjöström. Consistent solutions for cooperative games with externalities. Games and Economic Behavior, 43(2):196-213, 2003.

[45] K. Hyndman and D. Ray. Coalition formation with binding agreements. The Review of Economic Studies, 74(4):1125-1147, 2007.

[46] K. Hyndman and D. Ray. Coalition formation with binding agreements. The Review of Economic Studies, 74(4):1125-1147, 2007.

[47] S. Kakutani. A generalization of brouwers fixed point theorem. Duke Math. J., 8(3):457-459, 091941.

[48] E. Kalai and M. Smorodinsky. Other solutions to nash's bargaining problem. Econometrica: Journal of the Econometric Society, pages 513-518, 1975.

[49] Y. Kannai. The core and balancedness. Handbook of game theory with economic applications, 1:355-395, 1992.

[50] T. Kawamori and T. Miyakawa. Nash bargaining solution under externalities. Mathematical Social Sciences, 14:1-7, 2016.

[51] F. Klijn and E. Sánchez. Sequencing games without initial order. Mathematical Methods of Operations Research, 63(1):53-62, 2006.

[52] L. Á. Kóczy. A recursive core for partition function form games. Theory and Decision, 63:41-51, 2007.

[53] L. Á. Kóczy. Sequential coalition formation and the core in the presence of externalities. Games and Economic Behavior, 66(1):559-565, 2009.

[54] M. Le Breton, G. Owen, and S. Weber. Strongly balanced cooperative games. International Journal of Game Theory, 20(4):419-427, 1992.

[55] M. Maschler, E. Solan, and S. Zamir. Game theory (translated from the hebrew by ziv hellman and edited by mike borns). Cambridge University Press, Cambridge, pp. xxvi, 979:4, 2013.

[56] E. S. Maskin. Bargaining, Coalitions and Externalities. Working Paper, Institute for Adavanced Study, 2003. 
[57] T. Miyakawa. Note on the equal split solution in an n-person noncooperative bargaining game. Mathematical Social Sciences, 55(3):281-291, 2008.

[58] M. Montero. Proportional payoffs in legislative bargaining with weighted voting: a characterization. Quarterly Journal of Political Science, 12(3):325-346, 2017.

[59] G. Mosheiov. Scheduling problems with a learning effect. European Journal of Operational Research, 132(3):687-693, 2001.

[60] G. Mosheiov and J. B. Sidney. Scheduling with general job-dependent learning curves. European Journal of Operational Research, 147(3):665670, 2003.

[61] H. Moulin. Axioms of cooperative decision making. Cambridge University Press, 1988.

[62] M. Musegaas, P. E. Borm, and M. Quant. Step out-step in sequencing games. European Journal of Operational Research, 246(3):894-906, 2015 .

[63] R. B. Myerson. Values of games in partition function form. International Journal of Game Theory, 6(2):23-31, 1977.

[64] R. B. Myerson. Game theory: analysis of conflict. Harvard University Press, Cambridge, Massachusetts, 1991.

[65] J. F. Nash. The bargaining problem. Econometrica: Journal of the Econometric Society, pages 155-162, 1950.

[66] J. F. Nash. Two-person cooperative games. Econometrica: Journal of the Econometric Society, pages 128-140, 1953.

[67] P. Norman. Legislative bargaining and coalition formation. Journal of Economic Theory, 102(2):322-353, 2002.

[68] A. Okada. A noncooperative coalitional bargaining game with random proposers. Games and Economic Behavior, 16(1):97-108, 1996. 
[69] A. Okada. The nash bargaining solution in general n-person cooperative games. Journal of Economic Theory, 145(6):2356-2379, 2010.

[70] A. Okada. Coalitional bargaining games with random proposers: Theory and application. Games and Economic Behavior, 73(1):227-235, 2011.

[71] M. J. Osborne and A. Rubinstein. Bargaining and Markets. Academic Press, New York, 1990.

[72] D. Pérez-Castrillo and D. Wettstein. Bidding for the surplus: a noncooperative approach to the shapley value. Journal of Economic Theory, 100(2):274-294, 2001.

[73] D. Ray and R. Vohra. A theory of endogenous coalition structures. Games and Economic Behavior, 26(2):286-336, 1999.

[74] D. Ray and R. Vohra. Coalition formation. In Handbook of game theory with economic applications, volume 4, pages 239-326. Elsevier, 2015.

[75] A. Rubinstein. Perfect equilibrium in a bargaining model. Econometrica: Journal of the Econometric Society, pages 97-109, 1982.

[76] R. Selten and U. Leopold. Equilibrium point selection in a bargaining situation with opportunity costs. 1980.

[77] R. Serrano and R. Vohra. Non-cooperative implementation of the core. Social Choice and Welfare, 14(4):513-525, 1997.

[78] L. S. Shapley. Cores of convex games. International journal of game theory, 1(1):11-26, 1971.

[79] P. P. Shenoy. On coalition formation: a game-theoretical approach. International Journal of Game Theory, 8:133-164, 1979.

[80] M. Slikker. Balancedness of sequencing games with multiple parallel machines. Annals of Operations Research, 137(1):177-189, 2005.

[81] M. Slikker. Relaxed sequencing games have a nonempty core. Naval Research Logistics (NRL), 53(4):235-242, 2006. 
[82] J. M. Snyder Jr, M. M. Ting, and S. Ansolabehere. Legislative bargaining under weighted voting. American Economic Review, 95(4):981-1004, 2005.

[83] R. M. Thrall and W. F. Lucas. N-person games in partition function form. Naval Research Logistics (NRL), 10:281-298, 1963.

[84] B. v. Velzen and H. Hamers. On the balancedness of relaxed sequencing games. Mathematical Methods of Operations Research, 57(2):287-297, 2003.

[85] J. Von Neumann. Zur theorie der gesellschaftsspiele. Mathematische annalen, 100(1):295-320, 1928.

[86] J. Von Neumann and O. Morgenstern. Theory of games and economic behavior. Princeton University Press Princeton, 1944.

[87] D. Wang, M.-Z. Wang, and J.-B. Wang. Single-machine scheduling with learning effect and resource-dependent processing times. Computers \& Industrial Engineering, 59(3):458-462, 2010.

[88] Wikipedia contributors. Foxconn - Wikipedia, the free encyclopedia. https://en.wikipedia.org/w/index.php?title=Foxconn\& oldid=999552219, 2021.

[89] E. Winter. Voting and vetoing. American Political Science Review, 90(4):813-823, 1996.

[90] C.-C. Wu and W.-C. Lee. Single-machine scheduling problems with a learning effect. Applied Mathematical Modelling, 32(7):1191-1197, 2008.

[91] H. Yan. Noncooperative selection of the core. International Journal of Game Theory, 31(4):527-540, 2003.

[92] S.-S. Yi. Endogenous formation of economic coalitions: a survey of the partition function approach. Endogenous Formation of Economic Coalitions, Edward Elgar, Cheltenham, UK, pages 80-127, 2003. 
[93] H. Yildirim. Proposal power and majority rule in multilateral bargaining with costly recognition. Journal of Economic Theory, 136(1):167-196, 2007.

[94] C. Zhao, Q. Zhang, and H. Tang. Machine scheduling problems with learning effects. Dynamics of Continuous, Discrete and Impulsive Systems, Series A: Mathematical Analysis, 11(5-6):741-750, 2004. 



\section{Acknowledgements}

This thesis would not have been successfully completed without help of those who gave me guidance, support, and encouragement. Many thanks to a few people for their consistent and illuminating instruction, which like a beacon, lightened my path forward in the ocean of research.

My deepest gratitude goes first and foremost to Professor Hao Sun, my supervisor at NPU. During my doctor's career in China, Professor Hao Sun acted like a father providing all his love to the children. He supported me in research and taught me all valuable knowledge of game theory. Most importantly, he taught me how to be a good researcher by personally setting an example. Professor Hao Sun also treated me like a friend and was always glad to help to deal with my troubles in daily life. I remember when I suffered from depression from study pressure, he was always there with a sympathetic ear willing to listen to my heart, and patiently gave me great encouragement and psychological counseling. After that, I realized that Professor Hao Sun is a great teacher who really cares about his students, not only in research, but in every aspect.

I am also deeply indebted to Professor Marc Uetz, my supervisor at UT. He is really a kind person and helped me a lot while I was in the Netherlands. Honestly, I got great assistance from Professor Marc Uetz to adapt to the unfamiliar environment and blend into my surroundings in a new research group. He also provided valuable comments and suggestions to improve my papers. In every weekly meeting, Professor Marc Uetz always patiently listen to my thoughts, even if my English expression was not always optimal. He can point out the essential problem in my research and propose great suggestions. I 
also would like to thank him for his effort in making my graduation proceed smoothly, even though some progress was a little behind the schedule.

I also want to express my heartfelt thanks to Ruben Hoeksma, my cosupervisor, and Alexander Skopalik. They provided me with great help and offered me precious comments during the whole process of my research, without which the thesis would not be what it is now. Also, I owe many thanks to Marjo Mulder, the secretary of our department at UT, who has put much effort into organizing many interesting daily activities. She always took extra care of my issues whenever I turned to her for help.

Finally, I am very grateful to my family members, my parents, and my grandfather. Only with their selfless support, concern, and love, I could overcome some difficulties and pursue my study till now. Their loving considerations and help are the source of my strength.

Guangjing Yang

January 2021, Enschede 


\section{About the Author}

Guangjing Yang was born on April 1st, 1993 in Xiangyang city, Hubei province, P. R. of China. After finishing his elementary education in his home city, he became a student in Xiangyang No. 7 middle school. In 2008, he qualified and passed the independent enrollment of Xiangyang No. 5 high school.

After 3 years of high school education, In September 2011, he was admitted as a student in Information and Computing Science Major, Northwestern Polytechnical University. In 2015, with his second-place of his class, he was recommended for admission to become a five-year PhD student in the Department of Applied Mathematics (School of Mathematics and Statistics), Northwestern Polytechnical University, under the supervision of Professor Hao Sun. In March 2019, his research was sponsored by the China Scholarship Council, and obtained the qualification of becoming a joint $\mathrm{PhD}$ student at the University of Twente. In September 2019, he started his doctoral career as a $\mathrm{PhD}$ student under the supervision of Professor Marc Uetz. 